Linköping studies in science and technology. Thesis.

No. 1656

Licentiate's Thesis

\title{
Probabilistic modeling for positioning applications using inertial sensors
}

\author{
Manon Kok
}

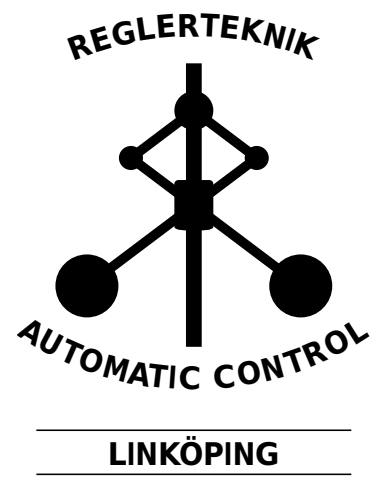

Division of Automatic Control

Department of Electrical Engineering

Linköping University, SE-581 83 Linköping, Sweden

http: //www. control.isy.liu.se

mankodisy.liu.se

Linköping 2014 
This is a Swedish Licentiate's Thesis.

Swedish postgraduate education leads to a Doctor's degree and/or a Licentiate's degree.

A Doctor's Degree comprises 240 ECTS credits (4 years of full-time studies).

A Licentiate's degree comprises 120 ECTS credits,

of which at least 60 ECTS credits constitute a Licentiate's thesis.

Linköping studies in science and technology. Thesis.

No. 1656

Probabilistic modeling for positioning applications using inertial sensors Manon Kok

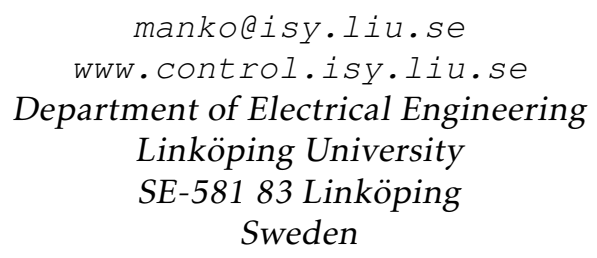

ISBN 978-91-7519-341-0ＩSSN 0280-7971ＩIU-TEK-LIC-2014:89

Copyright (C) 2014 Manon Kok

Printed by LiU-Tryck, Linköping, Sweden 2014 
To everyone who reads this 



\section{Abstract}

In this thesis, we consider the problem of estimating position and orientation (6D pose) using inertial sensors (accelerometers and gyroscopes). Inertial sensors provide information about the change in position and orientation at high sampling rates. However, they suffer from integration drift and hence need to be supplemented with additional sensors. To combine information from the inertial sensors with information from other sensors we use probabilistic models, both for sensor fusion and for sensor calibration.

Inertial sensors can be supplemented with magnetometers, which are typically used to provide heading information. This relies on the assumption that the measured magnetic field is equal to a constant local magnetic field and that the magnetometer is properly calibrated. However, the presence of metallic objects in the vicinity of the sensor will make the first assumption invalid. If the metallic object is rigidly attached to the sensor, the magnetometer can be calibrated for the presence of this magnetic disturbance. Afterwards, the measurements can be used for heading estimation as if the disturbance was not present. We present a practical magnetometer calibration algorithm that is experimentally shown to lead to improved heading estimates. An alternative approach is to exploit the presence of magnetic disturbances in indoor environments by using them as a source of position information. We show that in the vicinity of a magnetic coil it is possible to obtain accurate position estimates using inertial sensors, magnetometers and knowledge of the magnetic field induced by the coil.

We also consider the problem of estimating a human body's 6D pose. For this, multiple inertial sensors are placed on the body. Information from the inertial sensors is combined using a biomechanical model which represents the human body as consisting of connected body segments. We solve this problem using an optimization-based approach and show that accurate 6D pose estimates are obtained. These estimates accurately represent the relative position and orientation of the human body, i.e. the shape of the body is accurately represented but the absolute position can not be determined.

To estimate absolute position of the body, we consider the problem of indoor positioning using time of arrival measurements from an ultra-wideband (UWB) system in combination with inertial measurements. Our algorithm uses a tightlycoupled sensor fusion approach and is shown to lead to accurate position and orientation estimates. To be able to obtain position information from the UWB measurements, it is imperative that accurate estimates of the receivers' positions and clock offsets are known. Hence, we also present an easy-to-use algorithm to calibrate the UWB system. It is based on a maximum likelihood formulation and represents the UWB measurements assuming a heavy-tailed asymmetric noise distribution to account for measurement outliers. 



\section{Populärvetenskaplig sammanfattning}

I denna licentiatsavhandling betraktar vi problemet att skatta position och orientering med hjälp av tröghetssensorer (accelerometrar och gyroskop). Tröghetssensorer tillhandahåller information om förändringar i position och orientering vid höga samplingshastigheter. Nackdelen med denna typ av sensor är att skattningarna driver över tid (integrationsdrift) och behöver därför kompletteras med ytterligare sensorer. För att kombinera information från tröghetssensorer med information från andra sensorer använder vi probabilistiska modeller, både för sensorfusion och för sensorkalibrering.

Tröghetssensorer kan kompletteras med magnetometrar, som typiskt används för att erhålla riktningsinformation. Detta bygger på antaganden att det uppmätta magnetfältet är lika med ett konstant lokalt magnetfält och att magnetometern är korrekt kalibrerad. Närvaron av metalliska föremål i närheten av sensorn kommer att göra det första antagandet ogiltigt. Om det metalliska föremålet och magnetometern sitter ihop utan att kunna röra sig inbördes så kan magnetometern kalibreras med avseende på denna magnetiska störning. Efteråt kan mätningarna användas för riktningsskattning som om störningen inte var närvarande. I denna avhandling presenterar vi en praktisk algoritm för kalibrering av en magnetometer och visar att den leder till förbättrade skattningar av orientering. Ett alternativt tillvägagångssätt är att utnyttja närvaron av magnetiska störningar i inomhusmiljöer genom att använda dem som en källa till positionsinformation. Vi visar att i närheten av en magnetisk spole är det möjligt att erhålla precisa positionsskattningar med användning av tröghetssensorer, magnetometrar och kunskap om det magnetfält som induceras av spolen.

Vi ställer också upp problemet att skatta position och orientering hos en mänsklig kropp. För detta ändamål placeras flera tröghetssensorer på kroppen, och information från dessa kombineras med en biomekanisk modell som representerar den mänskliga kroppen. Denna modell består av kroppssegment som är knutna till varandra. Vi löser det resulterande problemet genom att använda en optimeringsbaserad metod vilket resulterar i korrekta relativa positions- och orienteringsskattningar. Detta betyder att formen på kroppen är rätt representerad men den absoluta positionen kan inte fastställas.

För att skatta den absoluta positionen av kroppen formulerar vi inomhuspositioneringsproblemet med hjälp av time of arrival mätningar från ett ultra-wideband (UWB) system i kombination med tröghetsmätningar. Vår algoritm använder ett angreppssätt baserat på tightly-coupled sensorfusion och leder till goda positionsoch orienteringsskattningar. För att kunna få positionsinformation från UWB mätningar är det nödvändigt att känna till UWB mottagarnas positioner och tidsförskjutningar. För detta ändamål presenterar vi en lättanvänd algoritm för att kalibrera ett UWB system. Den är baserad på en maximum likelihood formulering som modellerar bruset hos UWB mätningar med hjälp av en asymmetrisk fördelning med heavy tails för att hantera orimliga mätningar. 



\section{Acknowledgments}

The past 2.5 years have been an incredible journey in which I feel I have learned so much and I have met so many great people. The Automatic Control Group at Linköping University is a very inspiring environment and I am very grateful to Prof. Thomas Schön, Prof. Fredrik Gustafsson and Prof. Svante Gunnarsson for giving me the opportunity to join the group. Our head Prof. Svante Gunnarsson makes sure that there is always a good atmosphere and Ninna Stensgård is always there for help with administrative tasks. I would also like to thank Dr. Henk Luinge and Dr. Jeroen Hol. Without you I would not have started this journey.

My supervisor Prof. Thomas Schön is a great source of inspiration. Our meetings have become longer and longer over the past year but they have also become more and more interesting and I always feel inspired to go back to work afterwards. I would also like to thank my former colleagues at Xsens Technologies, specifically Dr. Henk Luinge and Dr. Jeroen Hol for our collaborations and for always welcoming me back whenever I am in Enschede.

This thesis has been proofread by Dr. Jeroen Hol, Dr. Gustaf Hendeby, Lic. Ylva Jung, Jonas Linder and my supervisors Prof. Thomas Schön and Prof. Fredrik Gustafsson. Your comments have been very valuable! The Swedish abstract would not be in such good shape without the help of Dr. Zoran Sjanic, Prof. Thomas Schön, soon-to-be-Lic. Johan Dahlin, Jonas Linder and Lic. Ylva Jung. Thanks a lot to you all!

Writing the thesis would not have been as easy without the IATEX template developed and maintained by Dr. Henrik Tidefelt and Dr. Gustaf Hendeby. Gustaf, your $\mathrm{LTT}_{\mathrm{E}} \mathrm{X}$ help, even late in the evenings and during the weekends is very much appreciated. I would also like to thank Dr. Daniel Petersson for introducing me to TikZ.

It would not have been as easy to move to another country were it not for all my colleagues. We have had a great time both at work and outside of work dancing bugg, playing and learning bridge, going to conferences, going out for a beer, having barbecues etc. I would like to thank my roommate Farzaneh Karami and my former roommate Dr. Zoran Sjanic for their company and for making our room a nice place to work in. I would also like to thank Lic. Sina Khoshfetrat Pakazad for always being the one to arrange fun things to do during weekends and evenings, Lic. Marek Syldatk for making our corridor more lively, Lic. Niklas Wahlström for nice times during conferences in Singapore and Vancouver, Lic. Ylva Jung for being a great friend and for convincing me to sometimes go and do some sports, soon-to-be-Lic. Johan Dahlin, for always being there to help me (and for defending his licentiate one week ahead of me so he always finds out how to do things before me :-) ), Jonas Linder for always being there to talk to and all other collegues for making sure that RT is such a nice place to work at and for all the fun things we do outside of work.

This work has been supported by MC Impulse, a European Commission, FP7 
research project and by CADICS, a Linnaeus Center funded by the Swedish Research Council (VR). The thesis would not have been possible without their financial support and I would like to hereby gratefully acknowledge both.

I would also like to thank my friends from outside our group. I have met a lot of international PhD students during for instance Swedish course, pedagogics course, our weekly lunches with our lunch group etc. It is great to meet people from all over the world and knowing so many people makes sure that Linköping feels like home.

Last but not least I would like to thank my parents, my sister and Mike. Mike, I know that it is a big change from living together to living $966 \mathrm{~km}$ away from each other. I hope you understand that I think it is worth it, thanks for your patience and support!

Finally, I would like to say that I am happy that the licentiate is only a half-way point. I am looking forward to the coming 2.5 years!

Linköping, May 2014

Manon Kok 


\section{Contents}

Notation

$\mathbf{X V}$

\section{Background}

1 Introduction 3

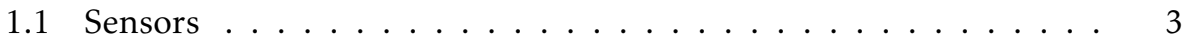

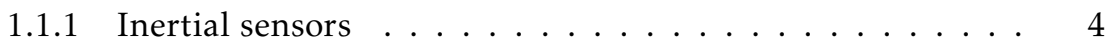

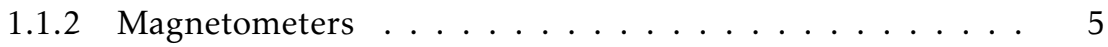

1.1 .3 Ultra-wideband . . . . . . . . . . . . 6

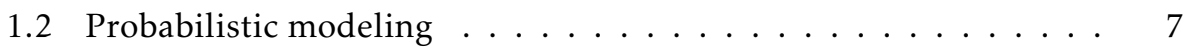

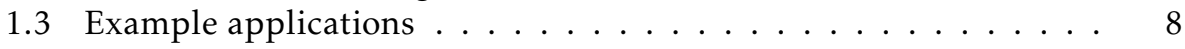

1.4 Thesis outline ........................ 9

2 Pose estimation using inertial sensors and magnetometers 15

2.1 Orientation representations . . . . . . . . . . . . 15

2.2 Extended Kalman filters for orientation estimation . . . . . . . . 17

2.2.1 The extended Kalman filter . . . . . . . . . . . . . 18

2.2.2 Modeling the orientation estimation problem . . . . . . 19

2.2.3 Quaternion states ..................... 20

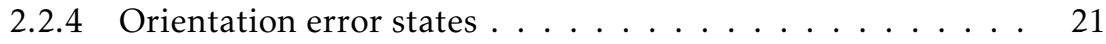

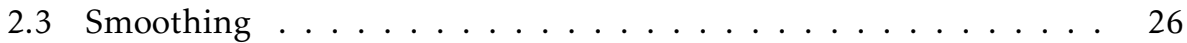

2.4 Particle filters ..................... 28

2.4.1 Representing a circle of possible sensor positions . . . . . 29

2.4 .2 Obtaining a point estimate . . . . . . . . 30

3 Sensor calibration $\quad 35$

3.1 Nonlinear optimization techniques . . . . . . . . . . . . . 36

3.2 Model parameters in the sensor models . . . . . . . . . . . . . . 39

3.3 Model parameters in a state-space model . . . . . . . . . . . . 40

4 Concluding remarks 43

4.1 Summary of the contributions ............... 43 
4.1 .1 Sensor calibration . . . . . . . . . . . . . . 43

4.1 .2 Pose estimation . . . . . . . . . . . . . 44

4.2 Future work . . . . . . . . . . . . . . . . 45

4.2.1 Sensor calibration . . . . . . . . . . . . . 45

4.2 .2 Pose estimation . . . . . . . . . . . . . 45

$\begin{array}{ll}\text { Bibliography } & 47\end{array}$

\section{Publications}

A Magnetometer calibration using inertial sensors 53

1 Introduction . . . . . . . . . . . . . . . 55

2 Problem formulation . . . . . . . . . . . . . 57

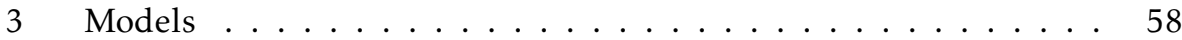

$3.1 \quad$ Dynamic model . . . . . . . . . . . . . . . 59

3.2 Accelerometer measurement model . . . . . . . . . . . 59

3.3 Magnetometer measurement model . . . . . . . . . . 59

3.4 Parameter vector . . . . . . . . . . . . 62

4 Finding good initial estimates .............. . . 63

$4.1 \quad$ Ellipse fitting . . . . . . . . . . . . 63

4.2 Determine misalignment of the inertial and magnetometer sensor axes .................... 65

5 Calibration algorithm .................... 66

6 Minimum rotation needed . . . . . . . . . . . . . 67

6.1 Identifiability analysis . . . . . . . . . . . . . 6 67 67

6.2 Quality of the estimates .............. 69

7 Experiments and results ................. 70

$7.1 \quad$ Experimental setup . . . . . . . . . . . . . . 70

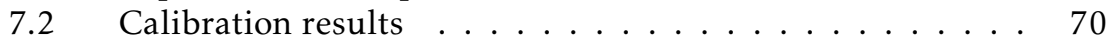

7.3 Heading estimation ..................... 74

8 Simulated heading accuracy . . . . . . . . . . . . . 75

9 Conclusions .................... 78

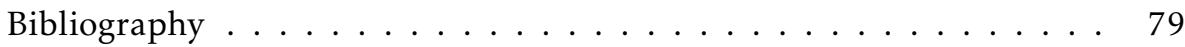

B Indoor positioning using ultra-wideband and inertial measurements 83

1 Introduction . . . . . . . . . . . . . . . . 85

2 Problem formulation ................ 87

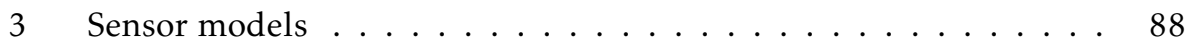

3.1 The ultra-wideband system . . . . . . . . . . . 88

3.2 Modeling the ultra-wideband measurements . . . . . . . 90

3.3 Modeling the inertial measurements . . . . . . . . . . . 91

4 Multilateration . . . . . . . . . . . . . . . . . . 92

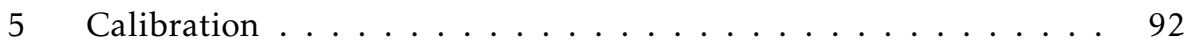

5.1 Computing an initial estimate . . . . . . . . . . 92

5.2 Resulting calibration algorithm . . . . . . . . . . 94

6 Sensor fusion . . . . . . . . . . . . . . . . . . . . 94 


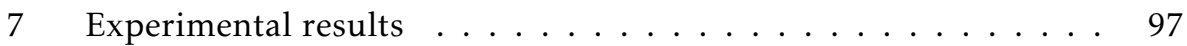

$7.1 \quad$ Calibration . . . . . . . . . . . . . . . 98

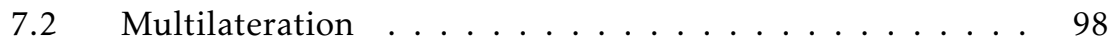

7.3 Pose estimation ....................... 100

8 Conclusions and future work . . . . . . . . . . . . . . . 103

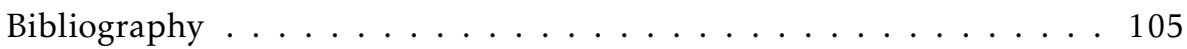

C An optimization-based approach to human body motion capture us$\begin{array}{lr}\text { ing inertial sensors } & 107\end{array}$

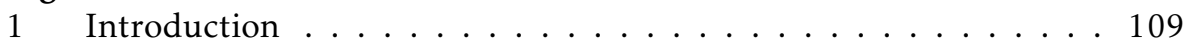

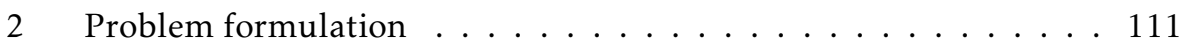

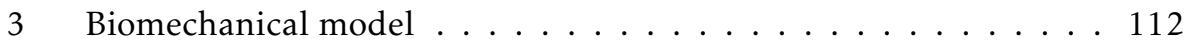

4 Dynamic and sensor models . . . . . . . . . . . . . 115

4.1 Dynamic model . . . . . . . . . . . . . 115

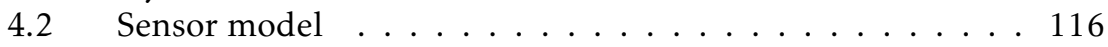

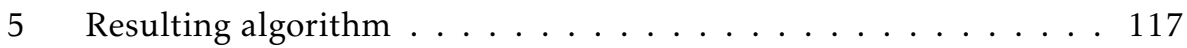

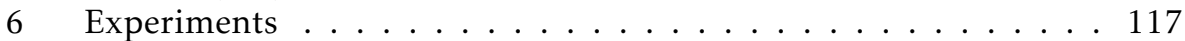

7 Conclusions and future work . . . . . . . . . . . . . 121

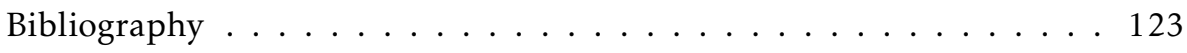

D MEMS-based inertial navigation based on a magnetic field map $\quad \mathbf{1 2 5}$

1 Introduction . . . . . . . . . . . . . . . 127

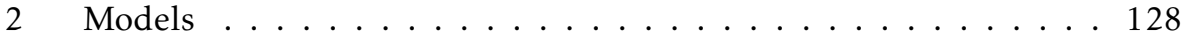

2.1 Dynamical model . . . . . . . . . . . . . . 129

2.2 Magnetometer measurement model . . . . . . . . . . 131

2.3 Some additional words about the magnetic field model . . 132

3 Computing the estimate ............... 132

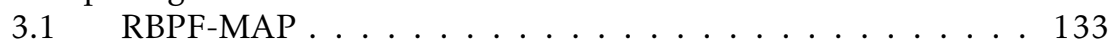

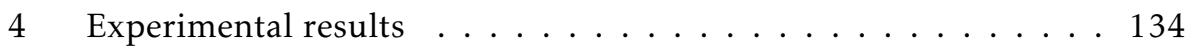

$4.1 \quad$ Experimental setup . . . . . . . . . . . . . . 134

$4.2 \quad$ Results ....................... 135

5 Conclusions and future work . . . . . . . . . . . . . 136

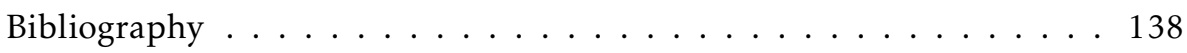





\section{Notation}

\section{SyMbOLS AND OPERATORS}

\begin{tabular}{cl}
\hline Notation & Meaning \\
\hline$n$ & Navigation frame \\
$x_{t}$ & Body frame \\
$x_{1: N}$ & State vector at time $t$ \\
$u_{t}$ & Known input vector at time $t$ \\
$y_{t}$ & Measurements at time $t$ \\
$y_{1: N}$ & Set of measurements from time $t=1$ to $t=N$ \\
$f_{t}(\cdot)$ & State update equation at time $t$ \\
$h_{t}(\cdot)$ & Measurement equation at time $t$ \\
$\hat{x}_{t \mid t}$ & State estimate at time $t$ given measurements up to and \\
$P_{t \mid t}$ & including time $t$ \\
$\theta$ & State covariance at time $t$ given measurements up to \\
$\hat{\theta}$ & and including time $t$ \\
$p(a \mid b)$ & Parameter vector \\
$p_{\theta}(b)$ & Parameter estimate \\
$\mathcal{N}\left(\mu, \sigma^{2}\right)$ & Probability of $b$ parametrized by $\theta$ \\
Cauchy $(\mu, \gamma)$ & Caussian distribution with mean $\mu$ and covariance $\sigma^{2}$ \\
$\emptyset$ & scale parameter $\gamma$ \\
$\in$ & Empty set \\
$A \subseteq B$ & Is a member of \\
$\mathbb{R}$ & Sis a subset of or is included in $B$ \\
$\arg \max$ & Maximizing argument \\
$\arg \min$ & Minimizing argument \\
$\|a\|_{2}$ & Two-norm of the vector $a$ \\
\hline & \\
&
\end{tabular}




\section{SyMbOLS AND OPERATORS}

\begin{tabular}{cl}
\hline Notation & Meaning \\
\hline $\mathcal{I}_{n}$ & $n \times n$ identity matrix \\
$\triangleq$ & Defined as \\
$\frac{\partial y}{\partial x}$ & Partial derivative of $y$ with respect to $x$ \\
$\operatorname{det} A$ & Determinant of the matrix $A$ \\
$A^{\top}$ & Transpose of the matrix $A$ \\
{$[a \times]$} & Cross product matrix of the vector $a$ \\
$A^{-1}$ & Inverse of the matrix $A$ \\
$\odot$ & Quaternion multiplication \\
$q^{\mathrm{L}}$ & Left quaternion multiplication of the quaternion $q$ \\
$q^{\mathrm{R}}$ & Right quaternion multiplication of the quaternion $q$ \\
$q_{v}$ & Vector part of the quaternion $q$ \\
\hline
\end{tabular}

\section{Abbreviations}

\begin{tabular}{cl}
\hline Abbreviation & Meaning \\
\hline BFGS & Broyden-Fletcher-Goldfarb-Shanno \\
EKF & Extended Kalman filter \\
GPS & Global positioning system \\
IMU & Inertial measurement unit \\
KF & Kalman filter \\
MAP & Maximum a posteriori \\
MEKF & Multiplicative extended Kalman filter \\
MEMS & Micro-machined electromechanical system \\
ML & Maximum likelihood \\
NLOS & Non-line-of-sight \\
PDF & Probability density function \\
PF & Particle filter \\
PF-MAP & Maximum a posteriori estimate for the particle filter \\
RBPF & Rao-Blackwellized particle filter \\
RBPF-MAP & Maximum a posteriori estimate for the Rao- \\
& Blackwellized particle filter \\
SLAM & Simultaneous localization and mapping \\
TOA & Time of arrival \\
UWB & Ultra-wideband \\
\hline
\end{tabular}


Part I

\section{Background}





\section{1}

Introduction

In this thesis, we consider the problem of estimating position and orientation using inertial sensors (accelerometers and gyroscopes). Throughout the thesis, the inertial measurements are used in combination with other sensors, namely magnetometers and time of arrival (TOA) measurements from an ultra-wideband (UWB) system. We also consider the problem of using multiple inertial sensors placed on the human body to estimate the body's position and orientation $(6 \mathrm{D}$ pose). Information from the inertial sensors is in that case combined using a biomechanical model which represents the human body as consisting of body segments that are attached to each other. To efficiently combine information from different sensors and different models, we rely on probabilistic models.

Part I of this thesis serves as background material to Part II in which four papers are presented. Hence, in Part I we will frequently refer to the different papers in Part II. In Section 1.1 of this chapter, we will first give a short description of the different sensors used throughout this thesis. Subsequently, the topic of probabilistic modeling will be introduced in Section 1.2. In the remainder we will discuss some example applications and summarize the contributions of this thesis.

\subsection{Sensors}

In this section we will introduce the sensors that are used throughout this thesis. In all four papers in Part II, our algorithms make use of inertial measurements from an inertial measurement unit (IMU). The IMUs we use are based on micromachined electromechanical system (MEMS) technology and are equipped with both inertial sensors (see Section 1.1.1) and with a three-axis magnetometer (see 

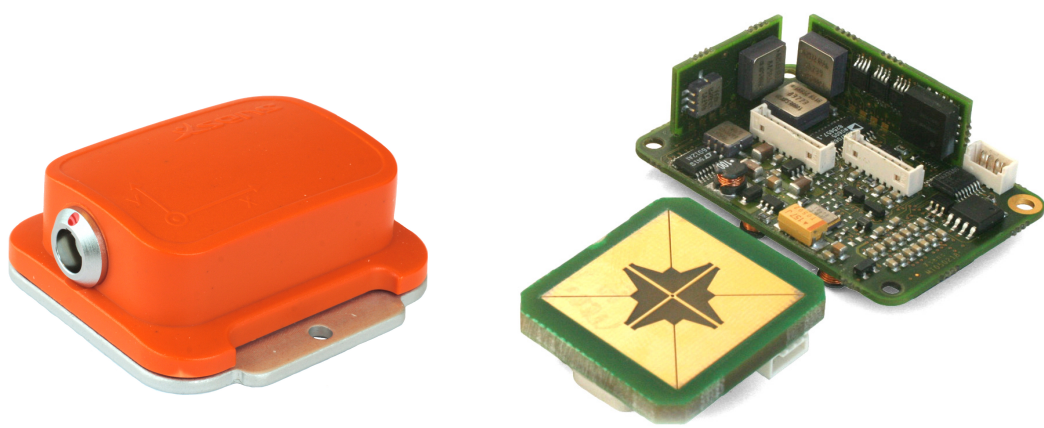

Figure 1.1: Example sensors. Left and right: an inertial measurement unit (IMU) with and without casing. Middle: an ultra-wideband (UWB) transmitter. By courtesy of Xsens Technologies.

Section 1.1.2). An example of an IMU can be found in Figure 1.1.

\subsubsection{Inertial sensors}

The term inertial sensor is used to denote the combination of a three-axis accelerometer and a three-axis gyroscope. A gyroscope measures the sensor's angular velocity, i.e. the rate of change of the sensor's orientation. Hence, integration of the gyroscope signals provides information about the orientation of the sensor.

An accelerometer measures the external specific force acting on the sensor. The specific force consists of both the sensor's acceleration and the earth's gravity. The earth's gravity is of the order of $9.81 \mathrm{~m} / \mathrm{s}^{2}$, while the sensor's acceleration is generally of much smaller magnitude. The accelerometer measurements will therefore typically consist of a large contribution from the earth's gravity and a relatively small contribution due to the motion of the sensor. After subtraction of the earth's gravity, double integration of the accelerometer signals provides information about the sensor position. To subtract earth's gravity, however, it is necessary that the orientation of the sensor is known. Hence, estimation of the sensor's position and orientation are inextricably linked when using inertial sensors. The combined estimation of both position and orientation is sometimes called pose estimation. The process of estimating position and orientation using inertial sensors is summarized in Figure 1.2.

The integration steps from angular velocity to rotation and acceleration to position introduce integration drift. Hence, errors in the measurements have a large impact on the quality of the estimated position and orientation using inertial sensors only. This is specifically the case for position, which relies both on double integration of the acceleration and on accurate orientation estimates to subtract the earth's gravity. Because of this, inertial sensors need to be supplemented with other sensors to lead to accurate position and orientation estimates. The inertial measurements can for instance be combined with TOA measurements from a UWB system. UWB will be introduced in Section 1.1.3. 


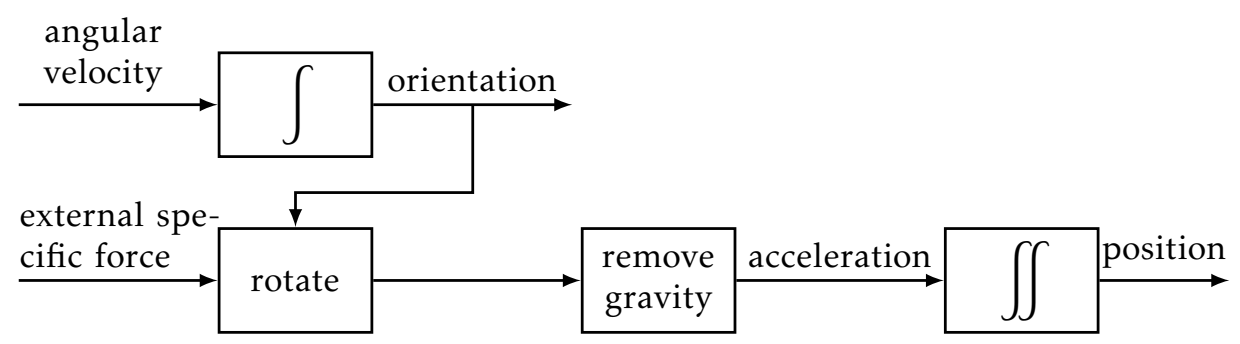

Figure 1.2: Schematic illustration of the process of determining position and orientation from the accelerometer measurements (external specific force) and the gyroscope measurements (angular velocity), assuming a known initial position and orientation.

In case we are interested in orientation estimation only, it is possible to use inertial sensors in combination with a magnetometer. For this, however, we need an additional model assumption concerning the acceleration. One can recognize that when the sensor is (almost) not accelerating, the accelerometer (almost) only measures the gravity. Using this model assumption, the accelerometer measurements can provide an estimate of the vertical direction (aligned with the gravity vector). The angle of deviation from the vertical is called the inclination. The accelerometer measurements can hence be said to stabilize the inclination estimates from the gyroscope. They do, however, not provide any information about the heading, i.e. the rotation around the vertical axis. Information about this can be obtained from magnetometers, which will be introduced in Section 1.1.2. Since IMUs often consist of both inertial sensors and magnetometers, it is for many applications possible to obtain accurate orientation estimates using an IMU.

\subsubsection{Magnetometers}

A magnetometer measures the strength and the direction of the magnetic field. In combination with inertial sensors, magnetometers typically serve the purpose of a compass and are used to determine the sensor's heading. This approach relies on the assumption that the magnetic field is at least locally constant and that it points in the direction of a local magnetic north. This is specifically the case when there are no magnetic objects in the vicinity of the sensor. In that case the magnetometer only measures the earth's magnetic field. Both the magnitude and the direction of the earth's magnetic field depend on the location on the earth, as depicted in Figure 1.3. However, the horizontal component of the magnetic field always points towards the earth's magnetic north.

Magnetometers typically provide accurate measurements of the magnetic field at high sampling rates. The measured magnetic field is, however, often not equal to the earth's magnetic field due to the presence of metallic objects in the vicinity of the sensor. The presence of objects causing magnetic disturbances is typically considered to be undesirable since they negatively affect the heading estimates. 


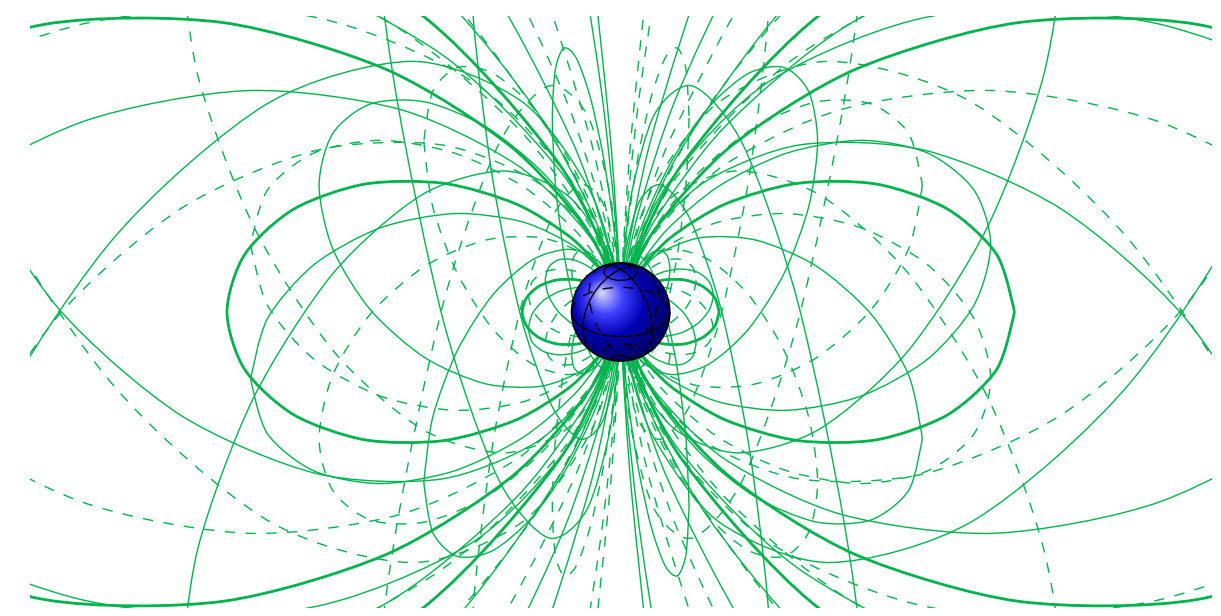

Figure 1.3: Schematic of the earth magnetic field lines (green) around the earth (blue).

However, if the metallic object is rigidly attached to the sensor the magnetometer can be calibrated for the presence of this disturbance. Afterwards, the measurements can be used for heading estimation as if the disturbance was not present. Example scenarios for which this calibration can be used are when a magnetometer is attached to e.g. a smartphone, a car or an aircraft. Magnetometer calibration is the topic of Paper A, where a practical magnetometer calibration algorithm is derived.

An alternative approach is to exploit the presence of magnetic disturbances in indoor environments by using them as a source of position information, see e.g. Angermann et al. (2012); Frassl et al. (2013). This approach assumes that knowledge of the magnetic field is represented as a map in which we want to localize the sensor. For instance, the strength and/or direction of the magnetic field at a specific location can be compared with a magnetic field map of the environment to estimate possible sensor locations. This is the topic of Paper D.

\subsubsection{Ultra-wideband}

A third type of measurements used in Part II of this thesis is based on TOA measurements from a UWB system. The UWB system consists of a number of stationary UWB receivers and a number of mobile transmitters, as depicted in Figure 1.4. The UWB transmitter (see also Figure 1.1) sends out a UWB pulse. The receivers measure the time of arrival of the pulse. Ideally, the time it takes for the pulse to reach the receivers is proportional to the distance between the transmitter and the receiver. However, due to multipath or non-line-of-sight (NLOS) conditions, the pulse can be delayed leading to a measurement outlier. In Paper B we consider the problem of indoor positioning using UWB measurements in combination with inertial measurements. The paper focuses on sensor fusion between 


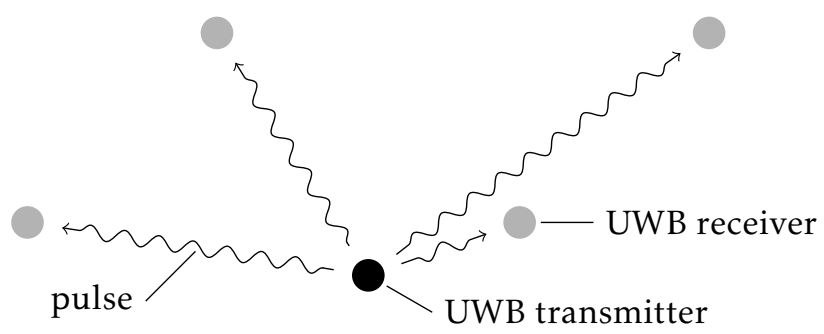

Figure 1.4: The UWB setup consists of a number of stationary receivers making TOA measurements of signal pulses originating from a mobile transmitter.

the UWB measurements and the inertial measurements. It also presents a calibration algorithm that determines the positions and clock offsets of the receivers and a novel approach to obtain position estimates using only the UWB measurements.

\subsection{Probabilistic modeling}

In this thesis we use measurements from the sensors discussed in Section 1.1 in combination with models to estimate the sensor's position and orientation. Both the measurements and the models provide uncertain information, for instance due to measurement noise or measurement outliers, but also due to model imperfections. Hence, we reason about our problem in terms of random variables with a probability density function (PDF). Combining information from different sensors based on a probabilistic framework is called sensor fusion, see e.g. Gustafsson (2012).

We typically describe our problems in the form of a state-space model,

$$
\begin{aligned}
x_{t+1} & =f_{t}\left(x_{t}, u_{t}, \theta, v_{t}\right), \\
y_{t} & =h_{t}\left(x_{t}, \theta, e_{t}\right),
\end{aligned}
$$

where $(1.1 \mathrm{a})$ is the dynamics or state update equation and $(1.1 \mathrm{~b})$ is the measurement equation. The dynamics model how the state changes over time, i.e. they describe the state $x$ at time $t+1$, denoted $x_{t+1}$, in terms of a possibly nonlinear and timevarying model $f_{t}(\cdot)$. The model $f_{t}(\cdot)$ depends on the state $x$, the input $u$ and the process noise $v$ at time $t$, and on a constant parameter vector $\theta$. The measurement equation models the measurements $y_{t}$ as a function $h_{t}(\cdot)$ of the state $x_{t}$, i.e. it describes which information about the state can be inferred from the measurements. The function $h_{t}(\cdot)$ also depends on a constant parameter vector $\theta$ and the measurement noise $e_{t}$. The noise terms $v_{t}$ and $e_{t}$ can reflect our confidence in the models and in the measurements, respectively. They can also be used to model different noise distributions to for instance take into account the presence of measurement outliers.

State-space models (1.1) are often used for state estimation, where we estimate 
the state $x_{1: N}=\left\{x_{1}, \ldots, x_{N}\right\}$. State estimation is often done using a maximum a posteriori (MAP) approach,

$$
\hat{x}_{1: N}^{\mathrm{MAP}}=\underset{x_{1: N}}{\arg \max } p\left(x_{1: N} \mid y_{1: N}\right),
$$

where $p(a \mid b)$ denotes the conditional probability of $a$ given $b$. Hence, the estimated state $x_{1: N}$ is chosen to be the one most likely from the measurements $y_{1: N}=\left\{y_{1}, \ldots, y_{N}\right\}$. Various techniques exist to obtain the MAP estimate. In Chapter 2 we will discuss background to the state estimation techniques that are used in the papers presented in Part II of this thesis.

In specific situations, the model parameters $\theta$ are unknown and need to be estimated from data. An example of this is sensor calibration where for instance the presence of an unknown measurement bias could be modeled as an unknown parameter in the measurement equation (1.1b). Estimation of parameters in a state-space model is also called grey-box system identification (Ljung, 1999; Bohlin, 2006). It can be done using maximum likelihood (ML) estimation,

$$
\hat{\theta}^{\mathrm{ML}}=\underset{\theta \in \Theta}{\arg \max } p_{\theta}\left(y_{1: N}\right),
$$

where $p_{\theta}(b)$ denotes the probability of $b$ parametrized by $\theta$. The parameter vector $\theta$ is an $n_{\theta}$-dimensional vector which can be limited to a subset $\Theta$ of $\mathbb{R}^{n_{\theta}}$, i.e. the optimization is performed over $\theta \in \Theta$ with $\Theta \subseteq \mathbb{R}^{n_{\theta}}$. The problem of sensor calibration will be discussed in more detail in Chapter 3 and will be the subject of Paper A and of part of Paper B.

\subsection{Example applications}

Position and orientation estimation is of interest for a wide range of applications. One can think of for instance aircraft or car localization, but also of pedestrian localization (Hol, 2011; Woodman, 2010; Grzonka, 2011; Callmer, 2013). For outdoor applications, it is typically possible to make use of measurements from a global positioning system (GPS). For indoor positioning, however, GPS signals are not available.

As discussed in Section 1.1.1, inertial sensors provide information about the change in orientation and position at high sampling rates. With the development of MEMS technology, small inertial sensors which can be worn on the human body have become available. This has applications in for instance pedestrian tracking (Woodman, 2010) which often focuses on estimating the position of firstresponders such as fire-fighters (Grzonka, 2011; Callmer, 2013). It also has applications for human body motion capture which is the subject of Paper C. There, a subject wears a suit with 17 IMUs on different body segments. The inertial measurements are used in combination with a biomechanical model to estimate the pose of the body. This biomechanical model is used to represent the assumption that the different body segments are (and remain) attached to each other. An example of pose estimates using inertial sensors is shown in Figure 1.5. The motion 


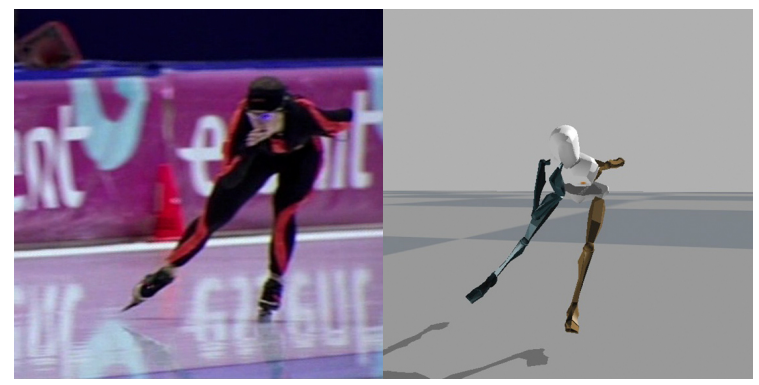

Figure 1.5: Example of inertial human body motion capture. Left: olympic and world champion speed skating Ireen Wüst wearing an inertial motion capture suit with 17 inertial sensors. Right: graphical representation of the estimated position and orientation of the body segments. By courtesy of Xsens Technologies.

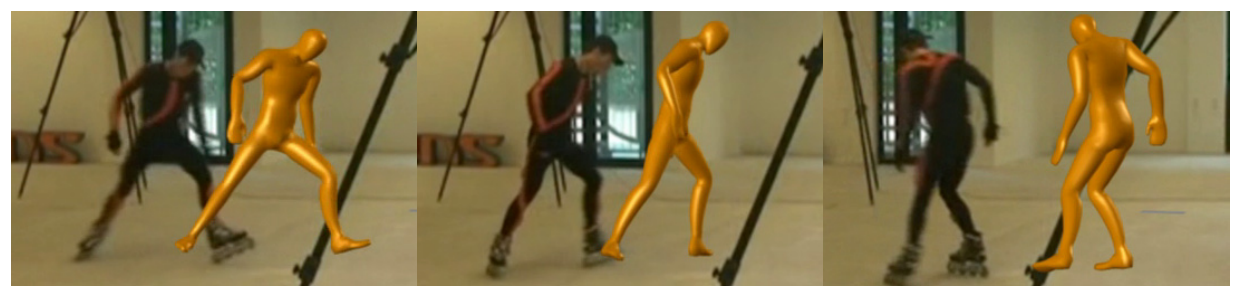

Figure 1.6: Example of inertial motion capture using 17 inertial sensors as well as 3 UWB transmitters on the head and on the feet. The estimated pose is shown in orange. By courtesy of Xsens Technologies.

capture suit can also be used in combination with UWB measurements. Paper B focuses on the use of UWB measurements and the sensor fusion of UWB measurements with inertial measurements. In Figures 1.6 and 1.7 a subject is shown who wears 17 inertial sensors as well as 3 UWB transmitters, on both his feet and his head.

\subsection{Thesis outline}

The thesis is divided into two parts, with edited versions of published and unpublished papers in Part II. In Part I, we will give background information relevant to the different papers.

\section{Part I - Background}

In Chapter 2, we describe the subject of pose estimation using inertial sensors and magnetometers. We focus on different algorithms/algorithm implementations to estimate the sensor's orientation. This serves as background material to Papers A, B and C. We also discuss some issues related to particle filtering for 


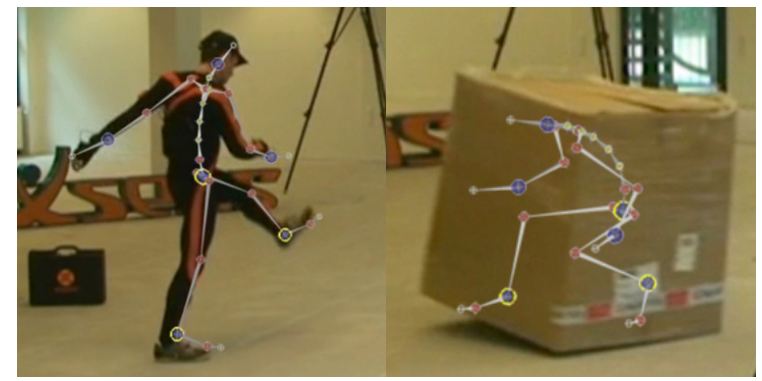

Figure 1.7: Example of inertial motion capture using 17 inertial sensors as well as 3 UWB transmitters on the head and on the feet. The estimated pose is overlaid on the body. As discussed in Section 1.1.3, UWB does not require line-of-sight. Hence, it is also possible to get good pose estimates when the subject is covered by for instance a box (right plot).

pose estimation using the magnetic field as a source of position information as in Paper D. In Chapter 3, we discuss the topic of sensor calibration. It provides background to the magnetometer calibration problem in Paper A and the UWB calibration algorithm presented in Paper B. Part I concludes with a summary of the contributions of the papers and a discussion of possible directions for future work.

\section{Part II - Publications}

Part II of the thesis consists of edited versions of four papers. These papers contain the following main contributions of this thesis:

- A novel magnetometer calibration algorithm which uses inertial sensors to calibrate the magnetometer for the presence of magnetic disturbances, for magnetometer sensor errors and for misalignment between the magnetometer and the inertial sensor axes [Paper A].

- A novel approach to combine inertial measurements with TOA measurements from a UWB system for indoor positioning. We present a tightlycoupled sensor fusion approach to combine the inertial measurements and the UWB measurements, an easy-to-use algorithm to calibrate the UWB setup and a novel multilateration approach to estimate the transmitter's position from the UWB measurements [Paper B].

- A novel inertial human body motion capture approach which solves the motion capture problem using an optimization-based approach [Paper C].

- A novel algorithm for $6 \mathrm{D}$ pose estimation where inertial measurements are complemented with magnetometer measurements assuming that a magnetic field map is known. In this approach, the magnetometer measurements are hence used as a source of position information [Paper D]. 
Below we provide a summary of each paper together with a discussion of the background and of the author's contributions.

\section{Paper A: Magnetometer calibration using inertial sensors}

Paper $\mathrm{A}$ is an edited version of

M. Kok and T. B. Schön. Magnetometer calibration using inertial sensors. Preprint, $2014 \mathrm{~b}$.

Earlier versions of this work were presented in

M. Kok and T. B. Schön. Maximum likelihood calibration of a magnetometer using inertial sensors. In Proceedings of the 19th World Congress of the International Federation of Automatic Control (accepted for publication), Cape Town, South Africa, August 2014a,

M. Kok, J. D. Hol, T. B. Schön, F. Gustafsson, and H. Luinge. Calibration of a magnetometer in combination with inertial sensors. In Proceedings of the 15th International Conference on Information Fusion, Singapore, July 2012.

Summary: In this work we present a practical calibration algorithm that calibrates a magnetometer using inertial sensors. The calibration corrects for magnetometer sensor errors, for the presence of magnetic disturbances and for misalignment between the magnetometer and the inertial sensor axes. It is based on a maximum likelihood formulation and is formulated as an offline method. It is shown to give good results using data from two different commercially available sensor units. Using the calibrated magnetometer measurements in combination with the inertial sensors to determine orientation, is shown to lead to significantly improved heading estimates.

Background and contributions: Before the author of this thesis started her work as a PhD student at Linköping University, she worked at Xsens Technologies. During this time she studied the topic of magnetometer calibration. Hence, the magnetometer calibration problem provided a good starting point for research during her PhD. A first paper on this subject has therefore been co-authored with Dr. Jeroen Hol and Dr. Henk Luinge from Xsens Technologies. Later work has mainly been done in cooperation with Prof. Thomas Schön. Dr. Henk Luinge and Laurens Slot from Xsens Technologies and Dr. Gustaf Hendeby from Linköping University have been so kind as to help in collecting the data sets presented in the paper. The author of this thesis has implemented the calibration algorithm and has written a major part of the paper.

\section{Paper B: Indoor positioning using ultra-wideband and inertial measurements}

Paper B is an edited version of

M. Kok, J. D. Hol, and T. B. Schön. Indoor positioning using ultrawideband and inertial measurements. Preprint, 2014b. 
Summary: In this work we present an approach to combine measurements from accelerometers and gyroscopes (inertial sensors) with time of arrival measurements from an ultra-wideband system for indoor positioning. Our algorithm uses a tightly-coupled sensor fusion approach and is shown to lead to accurate 6D pose (position and orientation) estimates as compared to data from an optical reference system. To be able to obtain position information from the ultra-wideband measurements, it is imperative that accurate estimates of the receivers' positions and clock offsets are known. Hence, we also present an easy-to-use algorithm to calibrate the ultra-wideband system. It is based on a maximum likelihood formulation and represents the ultra-wideband measurements assuming a heavytailed asymmetric noise distribution to account for measurement outliers. Using the heavy-tailed asymmetric noise distribution and the calibration results, it is shown that accurate position estimates can be obtained from the ultra-wideband measurements using a novel multilateration approach.

Background and contributions: The co-authors of this paper, Dr. Jeroen Hol and Prof. Thomas Schön, have been working on the subject of indoor positioning using ultra-wideband measurements and inertial measurements, resulting in the two papers Hol et al. $(2009,2010)$ and in the results presented in Hol (2011). The author of this thesis has extended the calibration and multilateration algorithms from Hol (2011); Hol et al. (2010) by assuming a heavy-tailed asymmetric distribution to represent the outliers in the ultra-wideband measurements. The presented sensor fusion results are based on previous results from Hol et al. (2009). The paper has been written together with Dr. Jeroen Hol.

\title{
Paper C: An optimization-based approach to human body motion capture using inertial sensors
}

Paper $\mathrm{C}$ is an edited version of

\begin{abstract}
M. Kok, J. D. Hol, and T. B. Schön. An optimization-based approach to human body motion capture using inertial sensors. In Proceedings of the 19th World Congress of the International Federation of Automatic Control (accepted for publication), Cape Town, South Africa, August 2014a.
\end{abstract}

Summary: In inertial human motion capture, a multitude of body segments are equipped with inertial measurement units, consisting of 3D accelerometers, 3D gyroscopes and 3D magnetometers. Relative position and orientation estimates can be obtained using the inertial data together with a biomechanical model. In this work we present an optimization-based solution to magnetometer-free inertial motion capture. It allows for natural inclusion of biomechanical constraints, for handling of nonlinearities and for using all data in obtaining an estimate. As a proof-of-concept we apply our algorithm to a lower body configuration, illustrating that the estimates are drift-free and match the joint angles from an optical reference system. 
Background and contributions: The co-authors Dr. Jeroen Hol and Prof. Thomas Schön came up with the idea of solving the human body motion capture problem as an optimization problem. The implementation of the optimization algorithm has been done using a framework developed by Xsens Technologies. With this framework, it is possible to define the optimization problem at a high level. The author of this thesis has been involved in developing and implementing the algorithm, in the data collection and has written a major part of the paper.

\section{Paper D: MEMS-based inertial navigation based on a magnetic field map}

Paper D is an edited version of

M. Kok, N. Wahlström, T. B. Schön, and F. Gustafsson. MEMS-based inertial navigation based on a magnetic field map. In Proceedings of the 38th International Conference on Acoustics, Speech, and Signal Processing (ICASSP), pages 6466-6470, Vancouver, Canada, May 2013.

Summary: This paper presents an approach for $6 \mathrm{D}$ pose estimation where MEMS inertial measurements are complemented with magnetometer measurements assuming that a model (map) of the magnetic field is known. The resulting estimation problem is solved using a Rao-Blackwellized particle filter. In our experimental study the magnetic field is generated by a magnetic coil giving rise to a magnetic field that we can model using analytical expressions. The experimental results show that accurate position estimates can be obtained in the vicinity of the coil, where the magnetic field is strong.

Background and contributions: The idea of looking into pose estimation using magnetometers as a source of position information was started through discussions with Dr. Slawomir Grzonka during the CADICS "Learning World Models" workshop in 2010 in Linköping. The experiments used in the paper were performed while the author of this thesis was working at Xsens Technologies. During this time, a first implementation of the pose estimation algorithm was made, using an extended Kalman filter. During the author's time at Linköping University, the work has been extended with an implementation using a Rao-Blackwellized particle filter. The author of this thesis wrote a major part of this paper.

\section{Publications of related interest, but not included in this thesis}

J. Kronander, J. Dahlin, D. Jönsson, M. Kok, T. B. Schön, and J. Unger. Real-time video based lighting using GPU raytracing. In Proceedings of the 2014 European Signal Processing Conference (EUSIPCO), Lisbon, Portugal, September 2014. (submitted, pending review).

N. Wahlström, M. Kok, T. B. Schön, and F. Gustafsson. Modeling magnetic fields using Gaussian processes. In Proceedings of the 38th International Conference on Acoustics, Speech, and Signal Processing (ICASSP), pages 3522-3526, Vancouver, Canada, May 2013. 



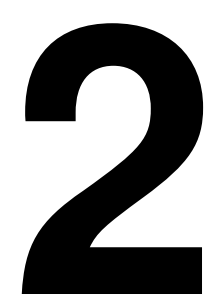

\section{Pose estimation using inertial sensors and magnetometers}

As discussed in Chapter 1, position and orientation estimation are closely related in the case of inertial sensors. Pose estimation denotes the simultaneous estimation of position and orientation. One can use standard estimation techniques for this. However, due to the nonlinear nature of the orientation and the different orientation representations, it is not obvious what is the best technique to use to estimate the orientation. In the different papers we use a variety of different techniques for orientation estimation, depending on the particular situation. In this chapter we will discuss a few different approaches and their pros and cons.

We start by introducing different representations of orientations in Section 2.1. Subsequently, two different extended Kalman filter (EKF) implementations are discussed in Section 2.2. EKFs can be used to solve the MAP problem (1.2) introduced in Chapter 1. Section 2.3 will introduce an alternative way of solving the MAP problem (1.2) using optimization techniques. In Section 2.4, some details with respect to particle filtering will be discussed.

\subsection{Orientation representations}

The orientation of an object is defined as the rotation between its coordinate frame with respect to a second coordinate frame. In this thesis we will mostly make use of the body coordinate frame $b$ and the navigation coordinate frame $n$. The body frame $b$ has its origin in the center of the accelerometer triad and its axes are aligned with the inertial sensor axes. The navigation frame $n$ is aligned with the earth's gravity and the local magnetic field.

Orientation can be represented in many different ways (Shuster, 1993). Perhaps the most intuitive representation is to make use of Euler angles. Rotation in 

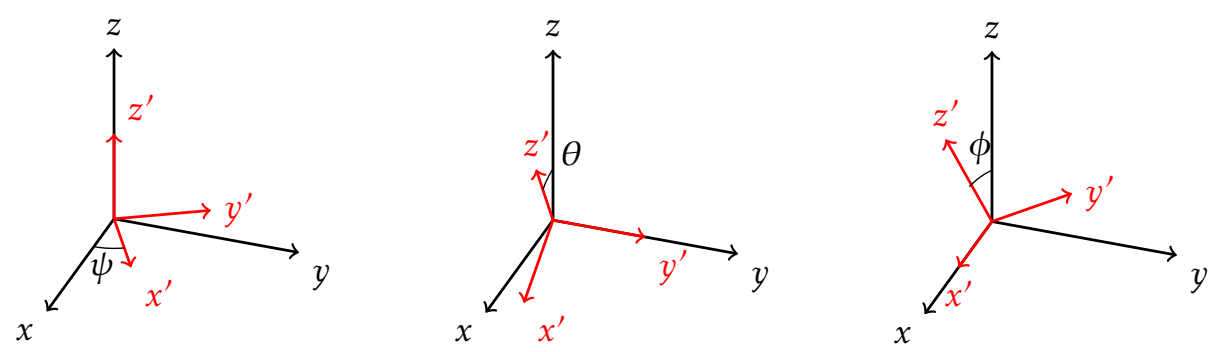

Figure 2.1: Definition of the Euler angles with left: rotation $\psi$ around the $z$-axis, middle: rotation $\theta$ around the $y$-axis and right: rotation $\phi$ around the $x$-axis.

terms of Euler angles is defined as a consecutive rotation around the three axes. We use the convention $(z, y, x)$ which first rotates around the $z$-axis, subsequently around the $y$-axis and finally around the $x$-axis. The rotations around the three axes, often denoted as the roll $\phi$, the pitch $\theta$ and the yaw $\psi$ angles, are depicted in Figure 2.1. Although Euler angles are an intuitive representation of orientation, they suffer from ambiguities. For instance, any addition of $2 \pi$ to the different angles results in the same orientation. Another ambiguity is sometimes called gimbal lock where certain rotation sequences lead to the same orientation, for instance the rotation $(0, \pi / 2, \pi)$ is equal to the rotation $(-\pi, \pi / 2,0)$.

An alternative way to represent orientation is to use rotation matrices where the rotation matrix representation of the Euler angle rotation $(\psi, \theta, \phi)$ is given by

$$
\begin{aligned}
R & =\left(\begin{array}{ccc}
1 & 0 & 0 \\
0 & \cos \phi & \sin \phi \\
0 & -\sin \phi & \cos \phi
\end{array}\right)\left(\begin{array}{ccc}
\cos \theta & 0 & -\sin \theta \\
0 & 1 & 0 \\
\sin \theta & 0 & \cos \theta
\end{array}\right)\left(\begin{array}{ccc}
\cos \psi & \sin \psi & 0 \\
-\sin \psi & \cos \psi & 0 \\
0 & 0 & 1
\end{array}\right) \\
& =\left(\begin{array}{ccc}
\cos \theta \cos \psi & \cos \theta \sin \psi & \sin \theta \\
\sin \phi \sin \theta \cos \psi-\cos \phi \sin \psi & \sin \phi \sin \theta \sin \psi+\cos \phi \cos \psi & \sin \phi \cos \theta \\
\cos \phi \sin \theta \cos \psi+\sin \phi \sin \psi & \cos \phi \sin \theta \sin \psi-\sin \phi \cos \psi & \cos \phi \cos \theta
\end{array}\right) .
\end{aligned}
$$

Rotation matrices are a useful orientation representation and they will frequently be used throughout this thesis. For orientation estimation purposes, however, rotation matrices are less suitable. The reason is that they would lead to a 9dimensional state vector subject to the following constraints

$$
R R^{\top}=R^{\top} R=\mathcal{I}_{3}, \quad \operatorname{det} R=1,
$$

where $\mathcal{I}_{3}$ denotes a $3 \times 3$ identity matrix.

A commonly used alternative orientation representation is that of unit quaternions. Quaternions were first introduced by Hamilton (1844) and are widely used in orientation estimation algorithms, see e.g. Kuipers (1999); Hol (2011). 
Quaternions use a 4-dimensional description of the orientation

$$
q=\left(\begin{array}{llll}
q_{0} & q_{1} & q_{2} & q_{3}
\end{array}\right)^{\top}=\left(\begin{array}{l}
q_{0} \\
q_{v}
\end{array}\right),
$$

with the constraint that $\|q\|_{2}=1$. The rotation matrix $R$ and the quaternion $q$ are related by

$$
\begin{aligned}
R & =q_{v} q_{v}^{\top}+q_{0}^{2} \mathcal{I}_{3}+2 q_{0}\left[q_{v} \times\right]+\left[q_{v} \times\right]^{2} \\
& =\left(\begin{array}{ccc}
2 q_{0}^{2}+2 q_{1}^{2}-1 & 2 q_{1} q_{2}-2 q_{0} q_{3} & 2 q_{1} q_{3}+2 q_{0} q_{2} \\
2 q_{1} q_{2}+2 q_{0} q_{3} & 2 q_{0}^{2}+2 q_{2}^{2}-1 & 2 q_{2} q_{3}-2 q_{0} q_{1} \\
2 q_{1} q_{3}-2 q_{0} q_{2} & 2 q_{2} q_{3}+2 q_{0} q_{1} & 2 q_{0}^{2}+2 q_{3}^{2}-1
\end{array}\right),
\end{aligned}
$$

where $\left[q_{v} \times\right]$ denotes the cross product matrix

$$
\left[q_{v} \times\right]=\left(\begin{array}{ccc}
0 & -q_{3} & q_{2} \\
q_{3} & 0 & -q_{1} \\
-q_{2} & q_{1} & 0
\end{array}\right) .
$$

Special quaternion algebra is available, see e.g. Kuipers (1999); Hol (2011). In this chapter, we will only introduce the quaternion algebra needed to derive the algorithms.

Note that a rotation is always represented from one coordinate frame to another. Hence, we use a double superscript on the rotation matrix $R$ and the quaternion $q$ as

$$
m^{\mathrm{n}}=R^{\mathrm{nb}} m^{\mathrm{b}},
$$

where $m^{\mathrm{b}}$ is a vector in the body frame $b$ and the rotation matrix $R^{\mathrm{nb}}$ rotates the vector to the navigation frame $n$. Equivalently,

$$
m^{\mathrm{b}}=\left(R^{\mathrm{nb}}\right)^{\mathrm{T}} m^{\mathrm{n}}=R^{\mathrm{bn}} m^{\mathrm{n}} .
$$

where a vector $m^{\mathrm{n}}$ in the navigation frame $n$ is rotated to the body frame $b$ using the rotation matrix $\left(R^{\mathrm{nb}}\right)^{\top}=R^{\mathrm{bn}}$.

\subsection{Extended Kalman filters for orientation estimation}

Orientation estimation is a state estimation problem, where the state $x_{1: N}$ in a state-space model (see (1.1)) is estimated from a time update and a measurement model. As discussed in Section 1.2, state estimation aims at obtaining a MAP estimate of the state. In the case of linear models this can be done using a Kalman filter (KF). KFs were first introduced by Kalman (1960) and are the best linear unbiased filters in the sense that they minimize the variance of the state estimation error. The EKF is an extension of the Kalman filter which makes the filter also applicable to nonlinear models. Unlike KFs, EKFs are not guaranteed to minimize the variance of the state estimation error. Actually, no guarantees for the quality of the EKF estimates can be given (Rawlings and Mayne, 2009). However, in cases where the model is not "too" nonlinear, they typically work well. EKFs are widely 
used due to their simplicity and computational efficiency, see e.g. Xsens Technologies B.V. (2014); Gustafsson (2012). For our case of estimating orientation using inertial measurements, EKFs are known to work quite well. The reason is that due to the high sampling rates of the IMU, each update in the EKF is typically not very nonlinear.

In the case of orientation estimation, the state in the EKF represents the orientation. Hence, a choice needs to be made which of the orientation representations (see Section 2.1) to use to represent the state. In this section, we will introduce two different EKF implementations for orientation estimation. To introduce the problem, in Section 2.2.1 we will first introduce the well-known EKF equations. Sections 2.2.3 and 2.2.4 will subsequently introduce EKF implementations to estimate orientation. The first uses a 4-dimensional quaternion state vector, the second uses a 3-dimensional state vector representing the orientation deviation from a linearization point. These discussions will focus on the simplest model to estimate orientations, i.e. we focus on an EKF implementation with only orientation states.

\subsubsection{The extended Kalman filter}

An EKF uses a nonlinear state-space model (1.1) as introduced in Section 1.2. We typically assume that the measurement noise is additive, and that both the process and the measurement noise are zero-mean Gaussian with constant covariance, i.e.

$$
\begin{aligned}
x_{t+1} & =f_{t}\left(x_{t}, u_{t}, v_{t}\right), \\
y_{t} & =h_{t}\left(x_{t}\right)+e_{t},
\end{aligned}
$$

with $v_{t} \sim \mathcal{N}(0, Q)$ and $e_{t} \sim \mathcal{N}(0, R)$.

The EKF estimates the state by performing a time update and a measurement update. The time update uses the model (2.8a) to "predict" the state to the next time step according to

$$
\begin{aligned}
& \hat{x}_{t+1 \mid t}=f_{t}\left(\hat{x}_{t \mid t}, u_{t}\right), \\
& P_{t+1 \mid t}=A_{t} P_{t \mid t} A_{t}^{\top}+G_{t} Q G_{t}^{\top},
\end{aligned}
$$

with

$$
A_{t}=\left.\frac{\partial f_{t}\left(x_{t}, u_{t}, v_{t}\right)}{\partial x_{t}}\right|_{x_{t}=\hat{x}_{t \mid t}, v_{t}=0}, \quad G_{t}=\left.\frac{\partial f_{t}\left(x_{t}, u_{t}, v_{t}\right)}{\partial v_{t}}\right|_{x_{t}=\hat{x}_{t \mid t}, v_{t}=0} .
$$

Here, $\hat{x}$ is used to distinguish the estimated state from the "true" state $x$. The matrix $P$ denotes the state covariance. The double subscripts on $\hat{x}_{t+1 \mid t}$ and $P_{t+1 \mid t}$ denote the state estimate and the state covariance at time $t+1$ given measurements up to time $t$. Similarly, $\hat{x}_{t \mid t}$ and $P_{t \mid t}$ denote the state estimate and the state covariance at time $t$ given measurements up to time $t$.

The measurement update uses the measurement model (2.8b) in combination 
with the measurements $y_{t}$ to update the "predicted" state estimate as

$$
\begin{aligned}
& \hat{x}_{t \mid t}=\hat{x}_{t \mid t-1}+P_{t \mid t-1} C_{t}^{\top}\left(C_{t} P_{t \mid t-1} C_{t}^{\top}+R\right)^{-1}\left(y_{t}-\hat{y}_{t \mid t-1}\right), \\
& P_{t \mid t}=P_{t \mid t-1}-P_{t \mid t-1} C_{t}^{\top}\left(C_{t} P_{t \mid t-1} C_{t}^{\top}+R\right)^{-1} C_{t} P_{t \mid t-1},
\end{aligned}
$$

with

$$
\hat{y}_{t \mid t-1}=h\left(\hat{x}_{t \mid t-1}\right), \quad C_{t}=\left.\frac{\partial h_{t}\left(x_{t}\right)}{\partial x_{t}}\right|_{x_{t}=\hat{x}_{t \mid t-1}} .
$$

Note that in (2.11) we have shifted our notation by one time step as compared to the notation in (2.9) to avoid cluttering the notation. The measurement update is often expressed in terms of the Kalman gain $K_{t}$, the residual $\varepsilon_{t}$ and the residual covariance $S_{t}$

$$
\varepsilon_{t}=y_{t}-\hat{y}_{t \mid t-1}, \quad S_{t}=C_{t} P_{t \mid t-1} C_{t}^{\top}+R, \quad K_{t}=P_{t \mid t-1} C_{t}^{\top} S_{t}^{-1} .
$$

The EKF iteratively performs a time update and a measurement update to estimate the state and the state covariance.

Design choices in the EKF are the choice of the state and of the dynamic and the measurement models. In Sections 2.2.2 - 2.2.4 we will focus on these design choices for the case of orientation estimation using inertial sensors and magnetometers. Hence, we will focus on the derivation of the models, the choice of the state $x$ and the derivation of the corresponding $f_{t}(\cdot), h_{t}(\cdot), A_{t}, C_{t}$, and $G_{t}$.

\subsubsection{Modeling the orientation estimation problem}

In this section we consider the problem of estimating orientation using inertial sensors and magnetometers. We use a measurement model where the gyroscope measurements $y_{\omega, t}$ are modeled as (Titterton and Weston, 1997)

$$
y_{\omega, t}=\omega_{t}+e_{\omega, t},
$$

where $\omega_{t}$ denotes the angular velocity and $e_{\omega, t} \sim \mathcal{N}\left(0, \Sigma_{\omega}\right)$. For simplicity we assume that the gyroscope measurements are bias-free.

The accelerometer measurements $y_{\mathrm{a}, t}$ are modeled as (Titterton and Weston, 1997)

$$
\begin{aligned}
y_{\mathrm{a}, t} & =R_{t}^{\mathrm{bn}}\left(a_{t}^{\mathrm{n}}-g^{\mathrm{n}}\right)+e_{\mathrm{a}, t} \\
& \approx-R_{t}^{\mathrm{bn}} g^{\mathrm{n}}+e_{\mathrm{a}, t},
\end{aligned}
$$

where $e_{\mathrm{a}, t} \sim \mathcal{N}\left(0, \Sigma_{\mathrm{a}}\right)$ and $R_{t}^{\mathrm{bn}}$ denotes the rotation from the navigation frame $n$ to the body frame $b$ at time $t$ as described in Section 2.1. As discussed in Chapter 1 , the accelerometer measures both the sensor's acceleration, denoted by $a_{t}^{\mathrm{n}}$ and the earth's gravity, denoted by $g^{\mathrm{n}}$. In the case of using only inertial sensors and magnetometers to estimate the orientation, it is necessary to stabilize the inclination by assuming something about the sensor's acceleration. A possible model for this is to assume that the mean of the acceleration is zero, as in Paper C and Luinge (2002). In this section and in Paper A we use a simpler model, where it is assumed that the acceleration $a_{t}^{\mathrm{n}}$ is approximately zero for all $t$. 
The magnetometer measurements $y_{\mathrm{m}, t}$ are modeled as

$$
y_{\mathrm{m}, t}=R_{t}^{\mathrm{bn}} m^{\mathrm{n}}+e_{\mathrm{m}, t},
$$

where $e_{\mathrm{m}, t} \sim \mathcal{N}\left(0, \Sigma_{\mathrm{m}}\right)$. The local magnetic field is denoted by $m^{\mathrm{n}}$. It is assumed to be constant and its horizontal component is assumed to be in the direction of the local magnetic north.

As discussed in Gustafsson (2012), it is possible to use the gyroscope measurements either as an input to the dynamic equation $(2.8 \mathrm{a})$ or as a measurement in (2.8b). In this thesis, we use an estimate of the angular velocity as a motion model for the orientation, i.e. we use the gyroscope measurements as an input to $(2.8 \mathrm{a})$. The noise $v_{t}$ in $(2.8 \mathrm{a})$ hence represents the measurement noise of the gyroscope.

\subsubsection{Quaternion states}

Using the model from Section 2.2.2, we will now derive an EKF to estimate the orientation using quaternions as a state vector. The state-space model (recall (2.8)) is for this case given by

$$
\begin{aligned}
q_{t+1}^{\mathrm{nb}} & =f_{t}\left(q_{t}^{\mathrm{nb}}, y_{\omega, t}, e_{\omega, t}\right), \\
y_{t} & =h_{t}\left(q_{t}^{\mathrm{nb}}\right)+e_{t},
\end{aligned}
$$

where $e_{\omega, t} \sim \mathcal{N}\left(0, \Sigma_{\omega}\right)$ and $e_{t} \sim \mathcal{N}(0, R)$. The measurement model uses the accelerometer and magnetometer measurement models (2.15) and (2.16).

The dynamic equation is given by (Gustafsson, 2012; Törnqvist, 2008)

$$
\begin{aligned}
q_{t+1}^{\mathrm{nb}} & =\exp \left(-\frac{T}{2} S\left(\omega_{t}\right)\right) q_{t}^{\mathrm{nb}} \\
& \approx\left(\mathcal{I}_{4}+\frac{T}{2} S\left(\omega_{t}\right)\right) q_{t}^{\mathrm{nb}} \\
& =\left(\mathcal{I}_{4}+\frac{T}{2} S\left(\hat{\omega}_{t}\right)\right) q_{t}^{\mathrm{nb}}+\frac{T}{2} \bar{S}\left(q_{t}^{\mathrm{nb}}\right) v_{t},
\end{aligned}
$$

where exp denotes the matrix exponential, $T$ denotes the sampling time and

$$
\hat{\omega}_{t}=y_{\omega, t}=\omega_{t}+e_{\omega, t} .
$$

The matrices $\bar{S}(q)$ and $S(\omega)$ are given by

$$
\bar{S}(q)=\left(\begin{array}{ccc}
-q_{1} & -q_{2} & -q_{3} \\
q_{0} & -q_{3} & q_{2} \\
q_{3} & q_{0} & -q_{1} \\
-q_{2} & q_{1} & q_{0}
\end{array}\right), \quad S(\omega)=\left(\begin{array}{cccc}
0 & -\omega_{1} & -\omega_{2} & -\omega_{3} \\
\omega_{1} & 0 & \omega_{3} & -\omega_{2} \\
\omega_{2} & -\omega_{3} & 0 & \omega_{1} \\
\omega_{3} & \omega_{2} & -\omega_{1} & 0
\end{array}\right) .
$$

To obtain (2.18b), a first order approximation is used. Subsequently, (2.18c) is obtained using the gyroscope measurement model (2.14). Note that without loss of generality we have changed the sign in front of the zero-mean Gaussian noiseterm in (2.14).

The state-space model (2.8) used to obtain the basic EKF equations, is therefore more explicitly given in terms of its dynamic equation (2.18) and its measure- 


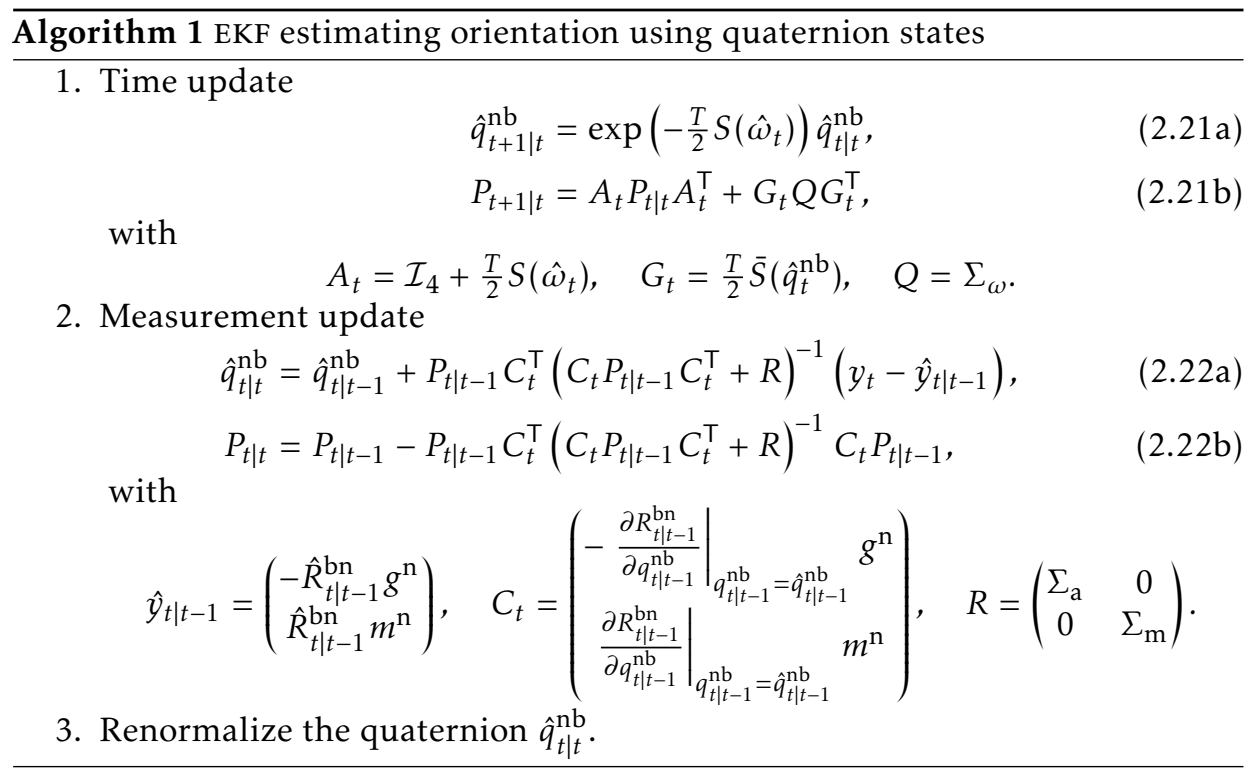

ment models (2.15) and (2.16). Using the results from Section 2.2.1, the EKF implementation for estimating orientation using quaternion states can be derived. It is given in Algorithm 1. Note that to avoid cluttering notation, the time indices are again shifted between the time and the measurement update.

Due to the norm 1 constraint of the quaternions, we expect the state covariance matrix $P$ in Algorithm 1 to be rank deficient. Due to linearization, however, the matrix $P$ in the EKF is typically not rank deficient. Hence, the actual interpretation of the covariance matrix is problematic. To avoid problems with a rank deficient state covariance matrix, in Section 2.2.4 we will derive a different EKF implementation.

\subsubsection{Orientation error states}

A second possible EKF implementation makes use of a 3-dimensional state vector $\eta_{t}$ representing the orientation deviation from a linearization point $\tilde{q}_{t}^{\text {nb }}$. This EKF implementation is sometimes referred to as a multiplicative EKF (MEKF) (Crassidis et al., 2007; Markley, 2003). Its derivation is slightly more involved than the one using quaternions. However, its implementation is computationally attractive since it only uses a 3-dimensional state (compared to the 4-dimensional state in Section 2.2.3). Furthermore, the interpretation of the state covariance is more intuitive since it is not expected to be rank-deficient as was the case for the quaternion implementation.

The linearization point $\tilde{q}_{t}^{\mathrm{nb}}$ is encoded using a unit quaternion. Defining without loss of generality ${ }^{1}$ that the orientation deviation is in the body frame $b$, the ori-

\footnotetext{
${ }^{1}$ A similar derivation can be done assuming an orientation deviation in the navigation frame $n$.
} 
entation $q_{t}^{\mathrm{nb}}$ can be defined in terms of a linearization point $\tilde{q}_{t}^{\mathrm{nb}}$ and the state $\eta_{t}^{\mathrm{b}}$ as

$$
q_{t}^{\mathrm{nb}}=\tilde{q}_{t}^{\mathrm{nb}} \odot \delta q\left(\eta_{t}^{\mathrm{b}}\right) .
$$

Here, $\odot$ denotes the quaternion multiplication defined as

$$
p \odot q \triangleq\left(\begin{array}{c}
p_{0} q_{0}-p_{v} \cdot q_{v} \\
p_{0} q_{v}+q_{0} p_{v}+p_{v} \times q_{v}
\end{array}\right),
$$

which can equivalently be written as the following matrix-vector multiplications

$$
p \odot q=\underbrace{\left(\begin{array}{cc}
p_{0} & -p_{v}^{\top} \\
p_{v} & p_{0} \mathcal{I}_{3}+\left[p_{v} \times\right]
\end{array}\right)}_{p^{\mathrm{L}}}\left(\begin{array}{l}
q_{0} \\
q_{v}
\end{array}\right)=\underbrace{\left(\begin{array}{cc}
q_{0} & -q_{v}^{\top} \\
q_{v} & q_{0} \mathcal{I}_{3}-\left[q_{v} \times\right]
\end{array}\right)}_{q^{\mathrm{R}}}\left(\begin{array}{l}
p_{0} \\
p_{v}
\end{array}\right) .
$$

The notation $\delta q(a)$ denotes the quaternion representation of a vector $a$ according to

$$
\delta q(a)=\left(\begin{array}{c}
\cos \frac{\|a\|}{2} \\
\frac{a}{\|a\|} \sin \frac{\|a\|}{2}
\end{array}\right) \approx\left(\begin{array}{c}
1 \\
\frac{a}{2}
\end{array}\right),
$$

where the second equality uses a first order approximation, assuming that the vector $a$ is small.

Based on (2.23), the dynamic and measurement models and the resulting EKF time and measurement update equations of the state $\eta_{t}^{\mathrm{b}}$ can be derived. In the remainder, the superscript $b$ will be omitted for brevity.

\section{Time update}

To determine the EKF time update equations, we need to derive the dynamic equation

$$
\eta_{t+1}=f_{t}\left(\eta_{t}, y_{\omega, t}, e_{\omega, t}\right) .
$$

To derive the dynamic model (2.27), we start from (2.23) for two different time steps

$$
\begin{aligned}
q_{t+1}^{\mathrm{nb}} & =\tilde{q}_{t+1}^{\mathrm{nb}} \odot \delta q\left(\eta_{t+1}\right), \\
q_{t}^{\mathrm{nb}} & =\tilde{q}_{t}^{\mathrm{nb}} \odot \delta q\left(\eta_{t}\right) .
\end{aligned}
$$

The dynamics of the orientation is defined in terms of the angular velocity $\omega_{t}$ as (see e.g. Shuster (1993); Hol (2011))

$$
q_{t+1}^{\mathrm{nb}}=q_{t}^{\mathrm{nb}} \odot \delta q\left(T \omega_{t}\right)
$$

where $\delta q\left(T \omega_{t}\right)$ is defined as in (2.26).

Comparing (2.28) and (2.29), we can use the gyroscope measurements to either update the linearization point $\tilde{q}_{t}^{\text {nb }}$ or to update the state $\eta_{t}$. Assuming that the 
gyroscope measurements are used to update the linearization point,

$$
\tilde{q}_{t+1}^{\mathrm{nb}}=\tilde{q}_{t}^{\mathrm{nb}} \odot \delta q\left(T \hat{\omega}_{t}\right),
$$

where $\hat{\omega}_{t}$ is defined in (2.19). Combining (2.28) - (2.30),

$$
\begin{aligned}
\delta q\left(\eta_{t+1}\right) & =\left(\tilde{q}_{t+1}^{\mathrm{nb}}\right)^{-1} \odot \tilde{q}_{t}^{\mathrm{nb}} \odot \delta q\left(\eta_{t}\right) \odot \delta q\left(T \omega_{t}\right) \\
& =\left(\delta q\left(T \hat{\omega}_{t}\right)\right)^{-1} \odot \delta q\left(\eta_{t}\right) \odot \delta q\left(T \omega_{t}\right) \\
& \approx\left(\begin{array}{c}
1 \\
-\frac{T}{2} \hat{\omega}_{t}
\end{array}\right)^{\mathrm{L}}\left(\begin{array}{c}
1 \\
\frac{T}{2} \omega_{t}
\end{array}\right)^{\mathrm{R}} \delta q\left(\eta_{t}\right) \\
& =\left(\begin{array}{c}
1 \\
-\frac{T}{2}\left(\omega_{t}+e_{\omega, t}\right)
\end{array}\right)^{\mathrm{L}}\left(\begin{array}{c}
1 \\
\frac{T}{2} \omega_{t}
\end{array}\right)^{\mathrm{R}} \delta q\left(\eta_{t}\right),
\end{aligned}
$$

where we used the definition (2.25), the gyroscope measurement model (2.14) and the definition of the inverse quaternion as

$$
q^{-1}=\left(\begin{array}{c}
q_{0} \\
-q_{v}
\end{array}\right)
$$

Note that in (2.32) we implicitly assume that the norm of the quaternion $q$ is equal to one. For a more general definition, see e.g. Hol (2011); Törnqvist (2008). Defining

$$
M \triangleq\left(\begin{array}{c}
1 \\
-\frac{T}{2}\left(\omega_{t}+e_{\omega, t}\right)
\end{array}\right)^{L}\left(\begin{array}{c}
1 \\
\frac{T}{2} \omega_{t}
\end{array}\right)^{R},
$$

and using the first-order approximation from (2.26), (2.31) can be written as

$$
\left(\begin{array}{c}
1 \\
\frac{\eta_{t+1}}{2}
\end{array}\right) \approx M\left(\begin{array}{c}
1 \\
\frac{\eta_{t}}{2}
\end{array}\right) \text {. }
$$

Hence, the dynamic model can be written as

$$
\eta_{t+1}=f_{t}\left(\eta_{t}, y_{\omega, t}, e_{\omega, t}\right) \approx 2 M_{21}+M_{22} \eta_{t},
$$

where $M_{21}$ and $M_{22}$ denote the $(2,1)$ and $(2,2)$ components of the matrix $M$, respectively, with

$$
\begin{aligned}
& M_{21}=-\frac{T}{2}\left(\omega_{t}+e_{\omega, t}\right)+\left(\mathcal{I}_{3}-\frac{T}{2}\left[\left(\omega_{t}+e_{\omega, t}\right) \times\right]\right) \frac{T}{2} \omega_{t} \\
& M_{22}=\frac{T^{2}}{4}\left(\omega_{t}+e_{\omega, t}\right) \omega_{t}^{\top}+\left(\mathcal{I}_{3}-\frac{T}{2}\left[\left(\omega_{t}+e_{\omega, t}\right) \times\right]\right)\left(\mathcal{I}_{3}-\frac{T}{2}\left[\omega_{t} \times\right]\right) .
\end{aligned}
$$

We assume that $\hat{\eta}_{t}=0$ and hence that the EKF time update affects the linearization point directly, i.e. $\hat{\eta}_{t+1}=0$ and

$$
\begin{aligned}
& \hat{q}_{t+1 \mid t}^{\mathrm{nb}}=\hat{q}_{t \mid t}^{\mathrm{nb}} \odot \delta q\left(T \hat{\omega}_{t}\right), \\
& P_{t+1 \mid t}=A_{t} P_{t \mid t} A_{t}^{\top}+G_{t} Q G_{t}^{\top},
\end{aligned}
$$


where $\hat{q}_{t \mid t}^{\mathrm{nb}}$ denotes the EKF estimate of the linearization point and

$$
\begin{aligned}
& A_{t}=\left.\frac{\partial f_{t}\left(\eta_{t}, y_{\omega, t}, e_{\omega, t}\right)}{\partial \eta_{t}}\right|_{\eta_{t}=\hat{\eta}_{t}, e_{\omega, t}=0} \\
&=\frac{T^{2}}{4} \hat{\omega}_{t} \hat{\omega}_{t}^{\top}+\left(\mathcal{I}_{3}-\frac{T}{2}\left[\hat{\omega}_{t} \times\right]\right)\left(\mathcal{I}_{3}-\frac{T}{2}\left[\hat{\omega}_{t} \times\right]\right) \\
& \approx \mathcal{I}_{3}-T\left[\hat{\omega}_{t} \times\right], \\
& G_{t}=\left.\frac{\partial f_{t}\left(\eta_{t}, y_{\omega, t}, e_{\omega, t}\right)}{\partial e_{\omega, t}}\right|_{\eta_{t}=\hat{\eta}_{t \mid t}, e_{\omega, t}=0} \\
&=-T \mathcal{I}_{3}+\frac{T^{2}}{4}\left[\hat{\omega}_{t} \times\right] \\
& \approx-T \mathcal{I}_{3} .
\end{aligned}
$$

Note that the approximations in (2.38) and (2.39) are not required, but are used for notational convenience.

\section{Measurement update}

In the measurement update of the EKF, the state $\eta_{t}$ is updated using the accelerometer and magnetometer measurements. Hence, the measurement equations (2.15) and (2.16) need to be formulated in terms of the state $\eta_{t}$. For this, it is possible to write

$$
\mathcal{R}\left(q_{t}^{\mathrm{nb}}\right)=\mathcal{R}\left(\tilde{q}_{t}^{\mathrm{nb}} \odot \delta q\left(\eta_{t}^{\mathrm{b}}\right)\right)=\mathcal{R}\left(\tilde{q}_{t}^{\mathrm{nb}}\right) \mathcal{R}\left(\delta q\left(\eta_{t}^{\mathrm{b}}\right)\right),
$$

where $\mathcal{R}(q)$ denotes the rotation matrix representation of the quaternion $q$. Using the relation between a rotation matrix and a quaternion (2.4) and the first order approximation (2.26) of the quaternion describing the orientation error,

$$
\begin{aligned}
\mathcal{R}\left(\delta q\left(\eta_{t}^{\mathrm{b}}\right)\right) & =(\delta q)_{v}(\delta q)_{v}^{\top}+\delta q_{0}^{2} \mathcal{I}_{3}+2 \delta q_{0}\left[(\delta q)_{v} \times\right]+\left[(\delta q)_{v} \times\right]^{2} \\
& =\frac{\eta_{t} \eta_{t}^{\top}}{4}+\mathcal{I}_{3}+\left[\eta_{t} \times\right]+\frac{1}{4}\left[\eta_{t} \times\right]^{2} \\
& \approx \mathcal{I}_{3}+\left[\eta_{t} \times\right],
\end{aligned}
$$

where for notational simplicity we have omitted the explicit dependence of $\delta q$ on $\eta_{t}^{\mathrm{b}}$ and the superscript $b$ on the $\eta_{t}$.

Using (2.40) and (2.41), the accelerometer measurement equation (2.15) can be written in terms of the state $\eta_{t}^{\mathrm{b}}$ as

$$
\begin{aligned}
y_{\mathrm{a}, t} & =\left(\mathcal{R}\left(q_{t}^{\mathrm{nb}}\right)\right)^{\top}\left(a_{t}^{\mathrm{n}}-g^{\mathrm{n}}\right)+e_{\mathrm{a}, t} \\
& \approx-\left(\mathcal{R}\left(q_{t}^{\mathrm{nb}}\right)\right)^{\top} g^{\mathrm{n}}+e_{\mathrm{a}, t} \\
& =-\left(\mathcal{R}\left(\delta q\left(\eta_{t}^{\mathrm{b}}\right)\right)\right)^{\top}\left(\mathcal{R}\left(\tilde{q}_{t}^{\mathrm{nb}}\right)\right)^{\top} g^{\mathrm{n}}+e_{\mathrm{a}, t} \\
& \approx-\left(\mathcal{I}_{3}-\left[\eta_{t}^{\mathrm{b}} \times\right]\right) \tilde{R}_{t}^{\mathrm{bn}} g^{\mathrm{n}}+e_{\mathrm{a}, t} \\
& =-\tilde{R}_{t}^{\mathrm{bn}} g^{\mathrm{n}}-\left[\tilde{R}_{t}^{\mathrm{bn}} g^{\mathrm{n}} \times\right] \eta_{t}^{\mathrm{b}}+e_{\mathrm{a}, t},
\end{aligned}
$$

where $\tilde{R}_{t}^{\mathrm{bn}}$ is the rotation matrix representation of $\tilde{q}_{t}^{\mathrm{bn}}$. 
The magnetometer measurement equation (2.16) can be written in terms of the state $\eta_{t}^{\mathrm{b}}$ as

$$
\begin{aligned}
y_{\mathrm{m}, t} & =\mathcal{R}\left(q_{t}^{\mathrm{nb}}\right)^{\top} m^{\mathrm{n}}+e_{\mathrm{m}, t} \\
& =\mathcal{R}\left(\delta q\left(\eta_{t}^{\mathrm{b}}\right)\right)^{\mathrm{T}}\left(\mathcal{R}\left(\tilde{q}_{t}^{\mathrm{nb}}\right)\right)^{\top} m^{\mathrm{n}}+e_{\mathrm{m}, t} \\
& \approx\left(\mathcal{I}_{3}-\left[\eta_{t}^{\mathrm{b}} \times\right]\right) \tilde{R}_{t}^{\mathrm{bn}} m^{\mathrm{n}}+e_{\mathrm{m}, t} \\
& =\tilde{R}_{t}^{\mathrm{bn}} m^{\mathrm{n}}-\left[\eta_{t}^{\mathrm{b}} \times\right] \tilde{R}_{t}^{\mathrm{bn}} m^{\mathrm{n}}+e_{\mathrm{m}, t} \\
& =\tilde{R}_{t}^{\mathrm{bn}} m^{\mathrm{n}}+\left[\tilde{R}_{t}^{\mathrm{bn}} m^{\mathrm{n}} \times\right] \eta_{t}^{\mathrm{b}}+e_{\mathrm{m}, t} .
\end{aligned}
$$

The EKF measurement update equations can hence be written as

$$
\begin{aligned}
& \hat{\eta}_{t}=P_{t \mid t-1} C_{t}^{\top}\left(C_{t} P_{t \mid t-1} C_{t}^{\top}+R\right)^{-1}\left(y_{t}-\hat{y}_{t \mid t-1}\right), \\
& \tilde{P}_{t \mid t}=P_{t \mid t-1}-P_{t \mid t-1} C_{t}^{\top}\left(C_{t} P_{t \mid t-1} C_{t}^{\top}+R\right)^{-1} C_{t} P_{t \mid t-1},
\end{aligned}
$$

with

$$
\begin{gathered}
y_{t}=\left(\begin{array}{c}
y_{\mathrm{a}, t} \\
y_{\mathrm{m}, t}
\end{array}\right), \quad \hat{y}_{t \mid t-1}=\left(\begin{array}{c}
-\hat{R}_{t \mid t-1}^{\mathrm{bn}} g^{\mathrm{n}} \\
\hat{R}_{t \mid t-1}^{\mathrm{bn}} m^{\mathrm{n}}
\end{array}\right), \\
C_{t}=\left(-\left[\hat{R}_{t \mid t-1}^{\mathrm{bn}} g^{\mathrm{n}} \times\right] \quad\left[\hat{R}_{t \mid t-1}^{\mathrm{bn}} m^{\mathrm{n}} \times\right]\right) .
\end{gathered}
$$

Note that we do not use a double subscript for the state $\hat{\eta}_{t}$ since the state is not updated in the time update of the filter. The covariance after the measurement update is denoted as $\tilde{P}_{t \mid t}$ since $P_{t \mid t}$ will be determined in the subsequent relinearization step.

\section{Relinearization}

After the measurement update, the orientation deviation $\hat{\eta}_{t}$ is non-zero. In obtaining the EKF time update equations, however, we assumed that the state $\eta_{t}$ was equal to zero. Hence, to not violate this assumption, we need to update the linearization point and reset the state after the measurement update. In our algorithm, we consider the relinearization as the "measurement update" for the linearization point, i.e. we assume that we update the estimate of the linearization point $\hat{q}_{t \mid t-1}^{\mathrm{nb}}$ to $\hat{q}_{t \mid t}^{\mathrm{nb}}$.

Defining the reset state as $\chi_{t}$, we model the relinearization as

$$
\chi_{t}=g_{t}\left(\eta_{t}\right) .
$$

Note the similarity with the EKF time update. Similar to the dynamic model $f_{t}(\cdot)$ we now have a function $g_{t}(\cdot)$ relating the current linearization point $\eta_{t}$ to a new linearization point $\chi_{t}$. The two linearization points are related by

$$
\hat{q}_{t \mid t}^{\mathrm{nb}} \odot \delta q\left(\chi_{t}\right)=\hat{q}_{t \mid t-1}^{\mathrm{nb}} \odot \delta q\left(\eta_{t}\right)
$$


and hence

$$
\delta q\left(\chi_{t}\right)=\underbrace{\left(\hat{q}_{t \mid t}^{\mathrm{nb}}\right)^{-1} \odot \hat{q}_{t \mid t-1}^{\mathrm{nb}}}_{\triangleq(\delta q(a))^{-1}} \odot \delta q\left(\eta_{t}\right) .
$$

Assuming that $\chi, \eta$ and $a$ are small, we can use the first-order approximation (2.26) and rewrite (2.48) as

$$
\left(\begin{array}{c}
1 \\
\frac{\chi_{t}}{2}
\end{array}\right)=\left(\begin{array}{c}
1 \\
-\frac{a}{2}
\end{array}\right)^{\mathrm{L}}\left(\begin{array}{c}
1 \\
\frac{\eta_{t}}{2}
\end{array}\right),
$$

and the relinearization model can be written as

$$
\chi_{t}=g_{t}\left(\eta_{t}\right) \approx-a+\left(\mathcal{I}_{3}-\frac{1}{2}[a \times]\right) \eta_{t} .
$$

Hence, the Jacobian of the relinearization can be determined as

$$
J_{t}=\left.\frac{\partial g_{t}\left(\eta_{t}\right)}{\partial \eta_{t}}\right|_{\eta_{t}=\hat{\eta}_{t}} \approx \mathcal{I}_{3}-\frac{1}{2}[a \times]
$$

Since the relinearization step is used to reset the state, we choose $\hat{\chi}_{t}=\left(\begin{array}{lll}0 & 0 & 0\end{array}\right)^{\top}$, i.e. $a=\hat{\eta}_{t}$. This leads to the following relinearization equations

$$
\begin{aligned}
& \hat{q}_{t \mid t}^{\mathrm{nb}}=\hat{q}_{t \mid t-1}^{\mathrm{nb}} \odot \delta q\left(\hat{\eta}_{t}\right), \\
& P_{t \mid t}=J_{t} \tilde{P}_{t \mid t} J_{t}^{\top}, \quad J_{t}=\mathcal{I}_{3}-\frac{1}{2}\left[\hat{\eta}_{t} \times\right] .
\end{aligned}
$$

The resulting EKF is summarized in Algorithm 2.

\subsection{Smoothing}

An alternative approach to obtain a MAP estimate of the state is to solve the problem (1.2) as a smoothing problem. Using such an approach, an estimate of the state vector $x_{1: N}$ using the measurements $y_{1: N}$ is obtained for instance using nonlinear optimization techniques (Boyd and Vandenberghe, 2004; Nocedal and Wright, 2006).

Optimization problems iteratively compute a smoothing estimate $\hat{x}_{1: N}$. An advantage of solving the state estimation problem using an optimization approach is that a relinearization is done after each iteration in the optimization problem. Hence, optimization problems can better handle nonlinearities than an EKF. It is also possible to include for instance constraints or non-Gaussian noise assumptions in the optimization problem.

The representation of the orientation in terms of an orientation deviation from a linearization point is particularly suitable for a smoothing implementation because of its low state dimension. Also, it does not require imposing a norm 1 constraint as is the case for quaternions.

As will be discussed in more detail in Chapter 3, solving optimization problems 


\section{Algorithm 2 EKF with orientation error states}

1. Time update

with

$$
\begin{aligned}
& \hat{q}_{t+1 \mid t}^{\mathrm{nb}}=\tilde{q}_{t \mid t}^{\mathrm{nb}} \odot \delta q\left(T \hat{\omega}_{t}\right), \\
& P_{t+1 \mid t}=A_{t} P_{t \mid t} A_{t}^{\top}+G Q G^{\top},
\end{aligned}
$$

2. Measurement update

$$
A_{t}=I_{3}-T\left[\hat{\omega}_{t} \times\right], \quad G=T \mathcal{I}_{3}, \quad Q=\Sigma_{\omega} .
$$

$$
\begin{aligned}
& \hat{\eta}_{t}=P_{t \mid t-1} C_{t}^{\top}\left(C_{t} P_{t \mid t-1} C_{t}^{\top}+R\right)^{-1}\left(y_{t}-\hat{y}_{t \mid t-1}\right) \\
& \tilde{P}_{t \mid t}=P_{t \mid t-1}-P_{t \mid t-1} C_{t}^{\top}\left(C_{t} P_{t \mid t-1} C_{t}^{\top}+R\right)^{-1} C_{t} P_{t \mid t-1},
\end{aligned}
$$

with

$$
\begin{aligned}
& y_{t}=\left(\begin{array}{c}
y_{\mathrm{a}, t} \\
y_{\mathrm{m}, t}
\end{array}\right), \quad \hat{y}_{t \mid t-1}=\left(\begin{array}{c}
-\hat{R}_{t \mid t-1}^{\mathrm{bn}} g^{\mathrm{n}} \\
\hat{R}_{t \mid t-1}^{\mathrm{bn}} m^{\mathrm{n}}
\end{array}\right),
\end{aligned}
$$

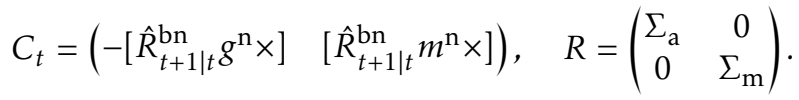

3. Relinearize

with

$$
\begin{aligned}
& \hat{q}_{t \mid t}^{\mathrm{nb}}=\hat{q}_{t \mid t-1}^{\mathrm{nb}} \odot\left(\begin{array}{c}
\cos \frac{\left\|\hat{\eta}_{t}\right\|}{2} \\
\frac{\hat{\eta}_{t}}{\left\|\hat{\eta}_{t}\right\|} \sin \frac{\left\|\hat{\eta}_{t}\right\|}{2}
\end{array}\right), \\
& P_{t \mid t}=J_{t} \tilde{P}_{t \mid t} J_{t}^{\top},
\end{aligned}
$$

$$
J_{t}=\mathcal{I}_{3}-\frac{1}{2}\left[\hat{\eta}_{t} \times\right]
$$




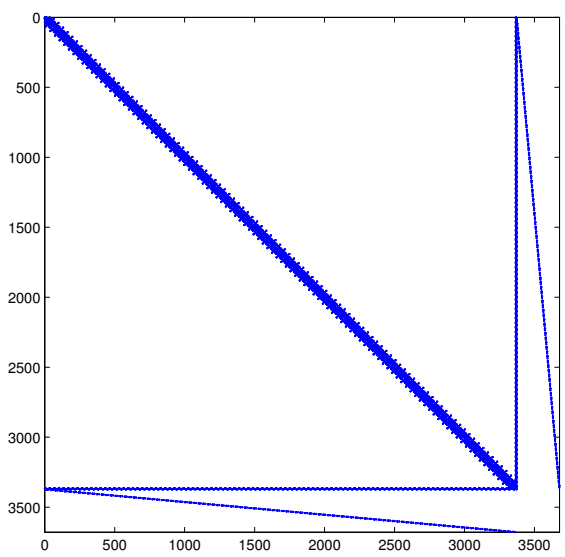

Figure 2.2: Sparseness pattern of the matrix that needs to be inverted to solve the motion capture problem in Paper $C$. The parts of the matrix that are non-zero are depicted in blue. The diagonal line represents the non-zero elements due to the dynamic and measurement models. The horizontal and vertical lines represent the sensor biases which we modeled as constants. The outermost diagonal lines represent the constraints based on the assumption that the body segments remain attached to each other at all times. Only $0.56 \%$ of the elements in the matrix are non-zero.

typically involves inversion of a matrix. For smoothing applications, this matrix grows with the number of measurements $y_{1: N}$ and the number of states $x_{1: N}$ and can hence be of fairly large dimension. Due to the specific structure of the state-space models, however, the number of non-zero elements in the matrix is typically relatively small, i.e. the matrix is sparse. This is because the state is modeled only in terms of the state at the previous time. Matrices that are sparse, and specifically matrices whose non-zero elements are ordered according to certain patterns, can be inverted efficiently (Boyd and Vandenberghe, 2004).

In Paper $C$ we use an optimization approach to solve an inertial motion capture problem. It estimates the body's 6D pose using information from a number of sensors attached to the body. The inertial measurements from these sensors are combined with the assumption that the body segments remain attached to each other at all times. This leads to a large optimization problem. However, as illustrated in Figure 2.2, the matrix that needs to be inverted to solve the optimization problem is very sparse and structured and can hence be inverted efficiently.

\subsection{Particle filters}

An alternative state estimation technique is the particle filter (PF) (Gordon et al., 1993; Doucet and Johansen, 2011). Contrary to EKFs, PFs do not rely on a linear approximation of the dynamic and measurement functions in the state-space 
model. Hence, they can successfully be used for state estimation using nonlinear models. PFs use $N$ particles to represent different hypotheses as

$$
\hat{p}^{N}\left(x_{t} \mid y_{1: t}\right)=\sum_{t=1}^{N} w_{t}^{i} \delta_{x_{t}^{i}}\left(x_{t}\right),
$$

where $w_{t}^{i}$ denotes the weight of particle $i$ at time $t$. In case the state-space model contains a conditionally linear Gaussian substructure, a Rao-Blackwellized particle filter (RBPF) (Schön et al., 2005) can be used instead. The RBPF treats the conditionally linear states using a KF, thereby reducing the dimension of the state vector used in the PF.

In this section we will not give a general description of a particle filter. Good descriptions can be found in for instance Doucet and Johansen (2011). Instead, we focus on two subproblems of relevance to Paper D. In Section 2.4.1 we will discuss the problem of using a PF to represent the state in a partially unobservable state-space model. In Section 2.4.2 we will discuss how a point estimate can be obtained from a PF describing a multimodal distribution.

\subsubsection{Representing a circle of possible sensor positions}

In Paper D, we use an RBPF to estimate the sensor's position and orientation using the magnetic field induced by a magnetic coil as a source of position information. Since the magnetic field strength is proportional to the distance to the coil, information about the magnetic field strength leads to information about the distance of the sensor to the coil. Hence, a sphere of possible position estimates is obtained. Based on the assumption that the inclination is known from the accelerometer measurements and assuming that the sensor is above the coil, the possible positions are reduced to a circle. Hence, the state-space model can be said to be unobservable, with the unobservable space in the shape of a circle. Although you would theoretically expect the particles in a PF to represent the unobservable space, i.e. you would expect a circle of particles, in practice this is not the case for a finite number of particles, as illustrated in Example 2.1.

\section{— Example 2.1: Particle filter estimating a partially unobservable state Consider the following state-space model

$$
\begin{aligned}
x_{t+1} & =x_{t}+v_{t}, \\
y_{t} & =\sqrt{x_{t, 1}^{2}+x_{t, 2}^{2}}+e_{t},
\end{aligned}
$$

where $x_{t}$ is a 2-dimensional state and $x_{t, i}$ denotes the $i^{\text {th }}$ component of $x_{t}$. We model $v_{t} \sim \mathcal{N}(0, Q)$ and $e_{t} \sim \mathcal{N}(0, R)$. For our simulations we choose $Q=1 \times$ $10^{-4} \mathcal{I}_{2}$ and $R=1 \times 10^{-4}$ and we use $N=500$ particles.

According to the dynamic model (2.57a), the state $x_{t}$ remains approximately constant. The measurement model $(2.57 \mathrm{~b})$ provides information about the state $x_{t}$, but any position on a circle with radius $y_{t}$ is equally likely. Hence, if we initialize the particles on a circle as in the left plot in Figure 2.3, we would expect the parti- 

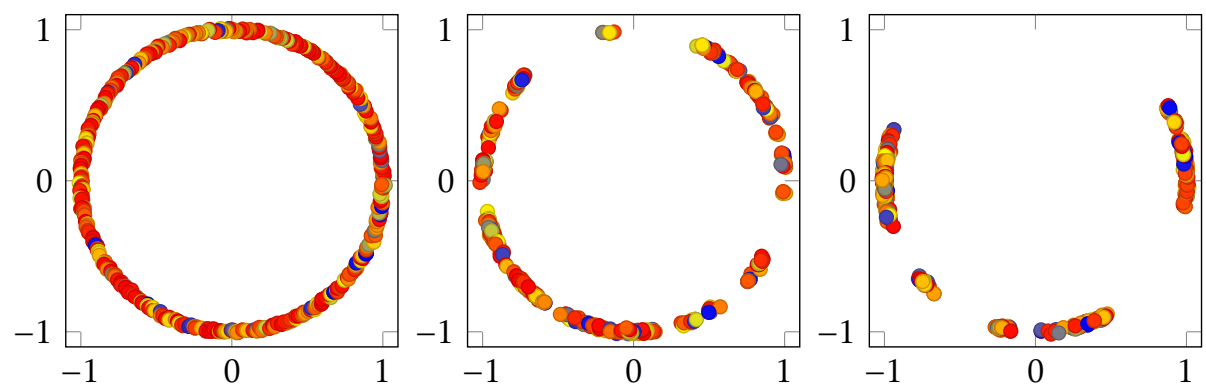

Figure 2.3: Particles of the PF used to estimate the state $x_{t}$ in Example 2.1. Left: the particles at $t=1$. The colors indicate the particles' weights. Middle: the particles at $t=50$. Right: particles at $t=500$.

cles to remain spread out over the circle. However, as depicted in the middle and right plots in Figure 2.3, as time progresses, the particles start clustering more and more on parts of the circle. How soon this clustering happens depends for instance on the particular choices of $Q, R$ and the number of particles $N$.

Note that although in Example 2.1 the particles at later time steps do not represent the whole circle, they do still give good estimates of $a$ solution. The hypotheses only don't include all solutions. The problem illustrated in Example 2.1 is a special case of particle degeneracy. As discussed in Lindsten (2013), after a certain time all particles will share a common ancestor at $t=1$. This results in all particles being clustered at one part of the circle.

\subsubsection{Obtaining a point estimate}

A particle filter uses $N$ particles and weights to represent the filtering density. Each particle is assigned a weight $w$ indicating how likely this hypothesis is. In practice, however, one often wants to represent the estimated state as a point estimate. For this, the weighted mean of the particles is commonly used. As argued in Driessen and Boers (2008); Saha et al. (2009), for some applications the weighted mean is not the most informative point estimate. An important example of this are multi-modal distributions where the point estimate based on the weighted mean is uninformative. For the Example 2.1, the weighted mean at the initial time point is clearly uninformative, since it is the circle's origin. Driessen and Boers (2008); Saha et al. (2009) therefore derive a MAP estimate for the particle filter, a so-called PF-MAP. Saha et al. (2013) derives a MAP estimate for a particle smoother. In Paper D we determine the position and orientation of an IMU in a magnetically disturbed environment using an RBPF. To determine a MAP estimate for the RBPF, the definition of the PF-MAP estimate needs to be extended. We will therefore in this section first introduce the PF-MAP as derived by Driessen and Boers (2008); Saha et al. (2009). Subsequently, we will introduce the RBPF-MAP. We use this RBPF-MAP in Paper D to determine a point estimate 
of the estimated sensor position to compare this to estimates from an optical reference system.

\section{PF-MAP}

In this section we will summarize the derivation of the PF-MAP introduced in Driessen and Boers (2008); Saha et al. (2009). The PF-MAP obtains a MAP estimate of the state at each time instance from the particles in the PF. The MAP estimate is defined as

$$
\hat{x}_{t \mid t}^{\mathrm{MAP}}=\underset{x_{t}}{\arg \max } p\left(x_{t} \mid y_{1: t}\right),
$$

i.e. it maximizes the posterior (filtering) density. Using Bayes' rule and the Markov property, this posterior density can be written as

$$
p\left(x_{t} \mid y_{1: t}\right)=\frac{p\left(y_{t} \mid x_{t}\right) p\left(x_{t} \mid y_{1: t-1}\right)}{p\left(y_{t} \mid y_{1: t-1}\right)} \propto p\left(y_{t} \mid x_{t}\right) p\left(x_{t} \mid y_{1: t-1}\right),
$$

where the denominator can be neglected since it is independent of $x_{t}$. The MAP estimate can hence be written as

$$
\hat{x}_{t \mid t}^{\mathrm{MAP}}=\underset{x_{t}}{\arg \max } p\left(y_{t} \mid x_{t}\right) p\left(x_{t} \mid y_{1: t-1}\right) .
$$

The predictive density $p\left(x_{t} \mid y_{1: t-1}\right)$ can be rewritten through marginalization as

$$
p\left(x_{t} \mid y_{1: t-1}\right)=\int p\left(x_{t} \mid x_{t-1}\right) p\left(x_{t-1} \mid y_{1: t-1}\right) d x_{t-1} .
$$

This density is in the particle filter approximated as

$$
p\left(x_{t} \mid y_{1: t-1}\right) \approx \sum_{j=1}^{N} p\left(x_{t} \mid x_{1: t-1}^{j}\right) w_{t-1}^{j},
$$

where $w_{t-1}^{j}$ denotes the weight of particle $j$ at time $t-1$. The MAP estimate can be obtained by substituting (2.62) into (2.60)

$$
\hat{x}_{t \mid t}^{\mathrm{MAP}}=\underset{x_{t}}{\arg \max } p\left(y_{t} \mid x_{t}\right) \sum_{j=1}^{N} p\left(x_{t} \mid x_{1: t-1}^{j}\right) w_{t-1}^{j} .
$$

To obtain the MAP estimate, (2.63) can be solved using optimization techniques as discussed in e.g. Boyd and Vandenberghe (2004); Nocedal and Wright (2006). An alternative is to use the PF-MAP. It approximates the MAP estimate as

$$
\hat{x}_{t \mid t}^{\mathrm{PF}-\mathrm{MAP}}=\underset{x_{t}^{i}}{\arg \max } p\left(y_{t} \mid x_{t}^{i}\right) \sum_{j=1}^{N} p\left(x_{t}^{i} \mid x_{1: t-1}^{j}\right) w_{t-1}^{j},
$$

i.e. it selects the particle with the highest density. Note that computation of the PF-MAP estimate is of the order of $N^{2}$ and it is hence quite expensive. 


\section{RBPF-MAP}

An RBPF splits the state vector in (2.8) into a nonlinear state $x_{t}^{\mathrm{n}}$ and a conditionally linear state $x_{t}^{1}$ as

$$
\begin{aligned}
x_{t+1}^{\mathrm{n}} & =f_{t}^{\mathrm{n}}\left(x_{t}^{\mathrm{n}}\right)+A_{t}^{\mathrm{n}}\left(x_{t}^{\mathrm{n}}\right) x_{t}^{1}+v_{t}^{\mathrm{n}}, \\
x_{t+1}^{1} & =f_{t}^{\mathrm{l}}\left(x_{t}^{\mathrm{n}}\right)+A_{t}^{1}\left(x_{t}^{\mathrm{n}}\right) x_{t}^{1}+v_{t}^{1}, \\
y_{t} & =h_{t}\left(x_{t}^{\mathrm{n}}\right)+C_{t}\left(x_{t}^{\mathrm{n}}\right) x_{t}^{1}+e_{t},
\end{aligned}
$$

with $Q=\left(\begin{array}{cc}Q^{\text {nn }} & Q^{\text {nl }} \\ \left(Q^{\text {nl }}\right)^{\top} & Q^{1 l}\end{array}\right)$. Note that for notational simplicity we here consider a slightly less general model than in (2.8). Note also that in this section we will switch notation from the rest of the chapter and use the superscript $n$ for "nonlinear" instead of "navigation frame". In this section we will derive the MAP for the RBPF in a similar way as the PF-MAP was derived in the previous section.

Following (2.58)-(2.60), and explicitly introducing the nonlinear and linear states,

$$
x_{t}=\left(\begin{array}{c}
x_{t}^{\mathrm{n}} \\
x_{t}^{1}
\end{array}\right)
$$

the MAP estimate can be written as

$$
\begin{aligned}
\hat{x}_{t \mid t}^{\mathrm{MAP}} & =\underset{x_{t}^{\mathrm{n}}, x_{t}^{1}}{\arg \max } p\left(x_{t}^{\mathrm{n}}, x_{t}^{1} \mid y_{1: t}\right) \\
& =\underset{x_{t}^{\mathrm{n}}, x_{t}^{1}}{\arg \max } p\left(y_{t} \mid x_{t}^{\mathrm{n}}, x_{t}^{1}\right) p\left(x_{t}^{\mathrm{n}}, x_{t}^{1} \mid y_{1: t-1}\right) .
\end{aligned}
$$

The predictive density can for the case of a RBPF be rewritten as

$$
p\left(x_{t}^{\mathrm{n}}, x_{t}^{1} \mid y_{1: t-1}\right)=\int p\left(x_{t}^{\mathrm{n}}, x_{t}^{1} \mid x_{t-1}^{1}, x_{t-1}^{\mathrm{n}}\right) p\left(x_{1: t-1}^{\mathrm{n}}, x_{t-1}^{1} \mid y_{1: t-1}\right) d x_{t-1}^{1} d x_{1: t-1}^{\mathrm{n}} \text {, }
$$

where, using Bayes' rule,

$$
p\left(x_{1: t-1}^{\mathrm{n}}, x_{t-1}^{1} \mid y_{1: t-1}\right)=p\left(x_{t-1}^{1} \mid x_{1: t-1}^{\mathrm{n}}, y_{1: t-1}\right) p\left(x_{1: t-1}^{\mathrm{n}} \mid y_{1: t-1}\right) .
$$

Here,

$$
p\left(x_{t-1}^{1} \mid x_{1: t-1}^{\mathrm{n}}, y_{1: t-1}\right)=\mathcal{N}\left(x_{t-1}^{1} ; \hat{x}_{t-1 \mid t-1}^{1}\left(x_{1: t-1}^{\mathrm{n}}\right), P_{t-1 \mid t-1}^{1}\left(x_{1: t-1}^{\mathrm{n}}\right)\right),
$$

and $p\left(x_{1: t-1}^{\mathrm{n}} \mid y_{1: t-1}\right)$ can be recognized as the particles' weights. This leads to

$$
\begin{aligned}
p\left(x_{t}^{\mathrm{n}}, x_{t}^{1} \mid y_{1: t-1}\right) \approx & \sum_{j=1}^{N} \int p\left(x_{t}^{\mathrm{n}, j}, x_{t}^{1, j} \mid x_{t-1}^{1, j}, x_{t-1}^{\mathrm{n}, j}\right) \\
& \mathcal{N}\left(x_{t-1}^{1} ; \hat{x}_{t-1 \mid t-1}^{1, j}, P_{t-1 \mid t-1}^{1, j}\right) w_{t-1}^{j} d x_{t-1}^{1},
\end{aligned}
$$


where we have introduced short-hand notation

$$
\begin{aligned}
& \hat{x}_{t-1 \mid t-1}^{1, j} \triangleq \hat{x}_{t-1 \mid t-1}^{1}\left(x_{1: t-1}^{\mathrm{n}, j}\right), \\
& P_{t-1 \mid t-1}^{1, j} \triangleq P_{t-1 \mid t-1}^{1}\left(x_{1: t-1}^{\mathrm{n}, j}\right) .
\end{aligned}
$$

The integral is computed similarly to the time update in a Kalman filter, see e.g. Lindsten and Schön (2013); Törnqvist (2008); Gustafsson (2012),

$$
p\left(x_{t}^{\mathrm{n}}, x_{t}^{1} \mid y_{1: t-1}\right) \approx \sum_{j=1}^{N} w_{t-1}^{j} \mathcal{N}\left(x_{t} ; \bar{x}_{t \mid t-1}^{j}, \bar{P}_{t \mid t-1}^{j}\right)
$$

with

$$
\begin{aligned}
& \bar{x}_{t \mid t-1}^{j}=\left(\begin{array}{l}
f_{t-1}^{\mathrm{n}}\left(x_{t \mid t-1}^{\mathrm{n}, j}\right) \\
f_{t-1}^{1}\left(x_{t \mid t-1}^{\mathrm{n}, j}\right)
\end{array}\right)+\left(\begin{array}{c}
A_{t-1}^{\mathrm{n}}\left(x_{t \mid t-1}^{\mathrm{n}, j}\right) \\
A_{t-1}^{1}\left(x_{t \mid t-1}^{\mathrm{n}, j}\right)
\end{array}\right) x_{t-1 \mid t-1}^{1, j}, \\
& \bar{P}_{t \mid t-1}^{j}=\left(\begin{array}{l}
A_{t-1}^{\mathrm{n}}\left(x_{t \mid t-1}^{\mathrm{n}, j}\right) \\
A_{t-1}^{1}\left(x_{t \mid t-1}^{\mathrm{n}, j}\right)
\end{array}\right) P_{t-1 \mid t-1}^{j}\left(\begin{array}{c}
A_{t-1}^{\mathrm{n}}\left(x_{t \mid t-1}^{\mathrm{n}, j}\right) \\
A_{t-1}^{1}\left(x_{t \mid t-1}^{\mathrm{n}, j}\right)
\end{array}\right)^{\top}+Q .
\end{aligned}
$$

Substituting (2.73) into (2.67), we obtain

$$
\hat{x}_{t \mid t}^{\mathrm{MAP}}=\underset{x_{t}^{\mathrm{n}}, x_{t}^{1}}{\arg \max } p\left(y_{t} \mid x_{t}^{\mathrm{n}}, x_{t}^{1}\right) \sum_{j=1}^{N} w_{t-1}^{j} \mathcal{N}\left(x_{t} ; \bar{x}_{t \mid t-1}^{j}, \bar{P}_{t \mid t-1}^{j}\right) .
$$

This can again be solved by any optimization technique, but it can also be solved approximately by optimizing over the finite set of particles, i.e.

$$
\hat{x}_{t \mid t}^{\mathrm{RBPF}-\mathrm{MAP}}=\underset{x_{t}^{\mathrm{n}, i}, x_{t}^{1, i}}{\arg \max } p\left(y_{t} \mid x_{t}^{\mathrm{n}, i}, x_{t}^{1, i}\right) \sum_{j=1}^{N} w_{t-1}^{j} \mathcal{N}\left(x_{t}^{i} ; \bar{x}_{t \mid t-1}^{j}, \bar{P}_{t \mid t-1}^{j}\right) .
$$

As can be seen, this is very similar to the PF-MAP results. This expression is used in Paper D. 



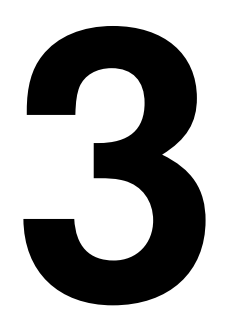

\section{Sensor calibration}

In the pose estimation algorithms discussed in Chapter 2, we implicitly assumed that the sensors were properly calibrated. In this chapter, we will instead focus on the problem of sensor calibration. Hence, we will assume that our models contain unknown model parameters that need to be determined from data. The process of determining a model from data is commonly referred to as system identification. In our problems, we typically know the model structure but to calibrate the sensor, certain model/calibration parameters need to be estimated. This is called grey-box system identification (Bohlin, 2006).

We formulate the sensor calibration problem as an ML problem, where based on $N$ measurements $y_{1: N}=\left\{y_{1}, \ldots, y_{N}\right\}$ we find the sensor calibration parameters $\theta$ that maximize the likelihood function (1.3). The ML problem (1.3) is repeated here for the reader's convenience,

$$
\hat{\theta}^{\mathrm{ML}}=\underset{\theta \in \Theta}{\arg \max } p_{\theta}\left(y_{1: N}\right),
$$

where $\Theta \subseteq \mathbb{R}^{n_{\theta}}$. Using the fact that the logarithm is a monotonic function, (3.1) has the following equivalent formulation,

$$
\hat{\theta}^{\mathrm{ML}}=\underset{\theta \in \Theta}{\arg \min }-\log p_{\theta}\left(y_{1: N}\right) .
$$

An example of a sensor calibration problem is the magnetometer calibration problem which is the topic of Paper A. In this paper, we calibrate the magnetometer for the presence of magnetic disturbances rigidly attached to the sensor, for magnetometer sensor errors and for misalignment between the magnetometer and the inertial sensor axes. In Chapter 2 we modeled the magnetometer measure- 
ments $y_{\mathrm{m}, t}$ as (2.16). For the reader's convenience, we repeat the model

$$
y_{\mathrm{m}, t}=R_{t}^{\mathrm{bn}} m^{\mathrm{n}}+e_{\mathrm{m}, t},
$$

where $R_{t}^{\mathrm{bn}}$ denotes the matrix rotating the local magnetic field $m^{\mathrm{n}}$ from the navigation frame $n$ to the body frame $b$ and $e_{\mathrm{m}, t}$ is assumed to be Gaussian noise. The measurement model (3.3a) was discussed in more detail in Section 2.2.2. In Paper A, we instead model the magnetometer measurements as

$$
y_{\mathrm{m}, t}=D R_{t}^{\mathrm{bn}} m^{\mathrm{n}}+o+e_{\mathrm{m}, t},
$$

where $D$ denotes the calibration matrix and $o$ denotes the offset vector. The calibration matrix $D$ and the offset vector $o$ are both part of the parameter vector $\theta$ determined in the calibration algorithm presented in Paper A. When the model parameters $D$ and $o$ have been determined, they can be used to correct the magnetometer measurements, i.e. they can be used to calibrate the magnetometer. Without magnetometer calibration, the orientation estimation algorithms discussed in Chapter 2 give inaccurate heading estimates for instance when the magnetometer is attached to a metallic object.

In this chapter, we will provide background to the sensor calibration problems in Papers A and B. In Section 3.1, we will first discuss the nonlinear optimization techniques we use to solve our sensor calibration problems, i.e. to obtain $\mathrm{ML}$ estimates of the sensor calibration parameters. In Sections 3.2 and 3.3, we subsequently discuss two different cases of sensor calibration. In Section 3.2 we discuss parameter estimation in a static sensor model. In Section 3.3, we instead focus on obtaining an ML estimate of the sensor calibration parameters in a state-space model. In that case, it is necessary to know the state to estimate the sensor calibration parameters. Hence, both the state and the calibration parameters need to be estimated to calibrate the sensor.

\subsection{Nonlinear optimization techniques}

To obtain an ML estimate, an optimization problem is solved which finds a locally minimizing argument (3.2). The specific form of the minimization problem depends on the model assumptions. However, in general it can be said that the parameters are chosen such that they best describe the data. Hence, defining the predicted measurements to be $\hat{y}(\theta)$, we try to minimize the difference

$$
\epsilon_{t}(\theta)=y_{t}-\hat{y}_{t}(\theta)
$$

according to a criterion which is based on the PDF $p_{\theta}\left(y_{1: N}\right)$. The function that needs to be minimized is denoted the cost function. Examples of cost functions can be found in Examples 3.1 and 3.2 for the cases of Gaussian and Cauchy distributed noise, respectively. The Cauchy distribution is a special case of the Student's-t distribution (Bishop, 2006). The Gaussian and Cauchy distributions will both be used in Part II of this thesis. More background about these distributions will be given in Section 3.2. 


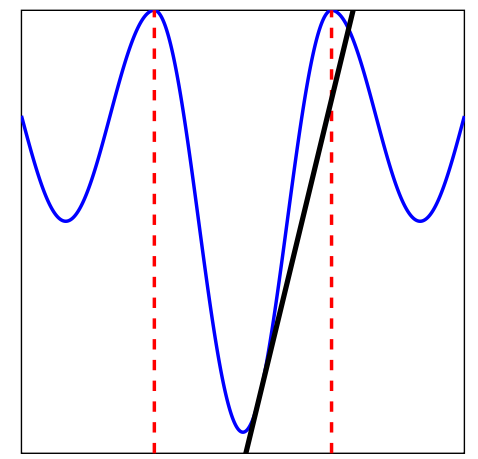

Figure 3.1: Blue: example of a cost function with multiple local minima. Black: example gradient. If we use the gradient information to find a direction in which the function decreases, any starting point between the two red dashed lines will lead us to the global minimum.

In our models, $\hat{y}_{t}$ typically depends nonlinearly on $\theta$. Hence, our problems typically have multiple (local) minima and proper initialization of the optimization problem is of essence. An example of a cost function is depicted in Figure 3.1. Since we aim at minimizing the cost function, it can intuitively be understood that we want our algorithm to step in a direction in which the objective function decreases. Hence, gradient information can be used to find a search direction. The cost function in Figure 3.1 has one global minimum, but also has two other local minima. If we use the gradient information to find a direction in which the function decreases, any starting point between the two red dashed lines will lead us to the global minimum. Starting points outside these two lines, however, would lead us to one of the other local minima instead. Initialization is therefore important for our problems to converge to the desired minimum and considerable effort is put into obtaining good initial estimates in Papers A and B.

Optimization algorithms based on only gradient information typically converge very slowly (Nocedal and Wright, 2006). Hence, most algorithms make use of both the gradient and an (approximate) Hessian to find the step direction. The general structure of the optimization algorithms used in this thesis is summarized in Algorithm 3. They start from an initial estimate $\hat{\theta}_{0}$ and update their estimate $\hat{\theta}$ until convergence.

Different types of algorithms use different (approximate) Hessians in Algorithm 3. A first type are the Newton methods which use the exact Hessian. A second commonly used approach uses a positive definite approximation of the Hessian as in (3.7b) and (3.10b). A third approach are the quasi-Newton methods. QuasiNewton methods estimate the Hessian based on the change of the gradient (Nocedal and Wright, 2006). Hence, they do not require the user to supply any analytical (approximate) Hessian. An example of a quasi-Newton method is the Broyden-Fletcher-Goldfarb-Shanno (BFGS) method which is used in Paper A to determine the magnetometer calibration parameters. 


\section{Example 3.1: Gaussian PDF with known and constant variance}

For the scalar case of a Gaussian PDF with a known and constant variance $\sigma^{2}$

$$
p_{\theta}\left(y_{1: N}\right)=\left(\frac{1}{\sqrt{2 \pi \sigma^{2}}}\right)^{N} \exp \left(-\sum_{t=1}^{N} \frac{\epsilon_{t}^{2}}{2 \sigma^{2}}\right) \text {, }
$$

with $\epsilon_{t}$ as defined in (3.4). Taking the logarithm of (3.5) and omitting constant terms, leads to the optimization problem

$$
\hat{\theta}^{\mathrm{ML}}=\underset{\theta \in \Theta}{\arg \min } V(\theta)=\underset{\theta \in \Theta}{\arg \min } \frac{1}{2} \sum_{t=1}^{N} \epsilon_{t}^{2} .
$$

Note that in our problems, (3.6) can not be solved explicitly since we assume that $\epsilon$ depends nonlinearly on $\theta$. To solve the optimization problem (3.6) we typically need the gradient $\nabla V$ and an approximate Hessian $H$ which in the case of a Gaussian are given by

$$
\begin{aligned}
\nabla V & =\sum_{t=1}^{N} \epsilon_{t} \frac{\partial \epsilon_{t}}{\partial \theta}, \\
H & \approx \sum_{t=1}^{N}\left(\frac{\partial \epsilon_{t}}{\partial \theta}\right)^{\top} \frac{\partial \epsilon_{t}}{\partial \theta} .
\end{aligned}
$$

Example 3.2: Cauchy PDF with known and constant scale parameter

For the scalar case of a Cauchy PDF with a known and constant scale parameter $\gamma$,

$$
p_{\theta}\left(y_{1: N}\right)=\prod_{t=1}^{N} \frac{1}{\pi \gamma^{2}}\left(1+\left(\frac{\epsilon_{t}}{\gamma}\right)^{2}\right)^{-1} \text {. }
$$

Taking the logarithm of (3.8) and omitting constant terms, leads to the optimization problem

$$
\hat{\theta}^{\mathrm{ML}}=\underset{\theta \in \Theta}{\arg \min } V(\theta)=\underset{\theta \in \Theta}{\arg \min } \sum_{t=1}^{N} \log \left(1+\left(\frac{\epsilon_{t}}{\gamma}\right)^{2}\right) .
$$

The gradient $\nabla V$ and a positive definite approximation of the Hessian are given by

$$
\begin{aligned}
\nabla V & =\sum_{t=1}^{N} 2 \frac{\frac{\partial \epsilon_{t}}{\partial \theta} \epsilon_{t}}{\gamma^{2}+\epsilon_{t}^{2}}, \\
H & \approx \sum_{t=1}^{N} 2 \frac{\left(\frac{\partial \epsilon_{t}}{\partial \theta}\right)^{\top} \frac{\partial \epsilon_{t}}{\partial \theta}}{\gamma^{2}+\epsilon_{t}^{2}} .
\end{aligned}
$$




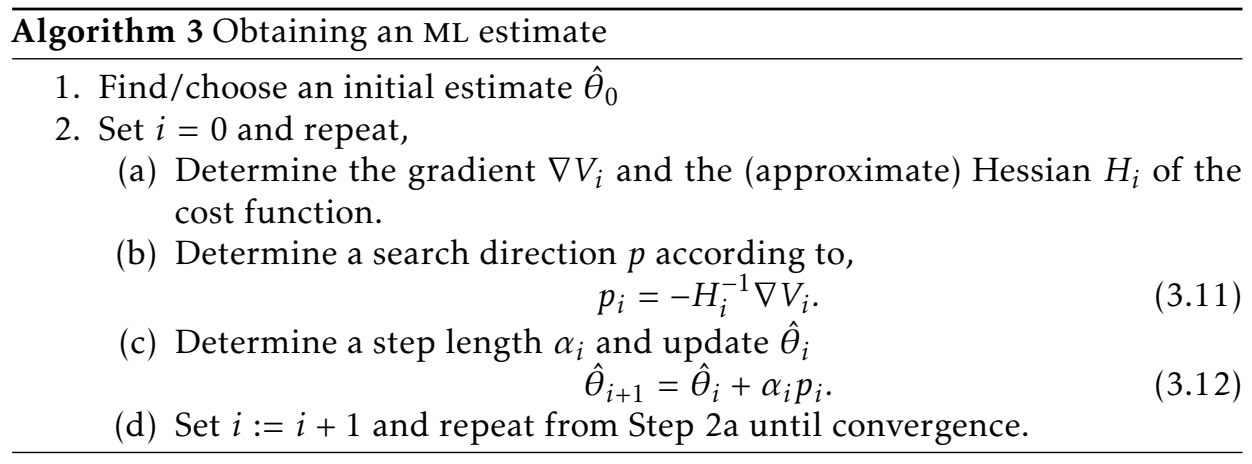

\subsection{Model parameters in the sensor models}

In this section, we focus on determining model parameters in static sensor models. We model the measurements as

$$
y_{t}=h_{t}(\theta)+e_{t}
$$

where $h_{t}(\cdot)$ is a possibly nonlinear function of the parameters $\theta$ and the noise $e_{t}$ is assumed to be additive.

An example of such a model is used in Paper B where we model the UWB measurements as

$$
y_{\mathrm{u}, m k}=\tau_{k}+\left\|r_{m}^{\mathrm{n}}-t_{k}^{\mathrm{n}}\right\|_{2}+\Delta \tau_{m}+e_{\mathrm{u}, m k},
$$

where $\tau_{k}$ is the time of transmission of pulse $k, t_{k}^{\mathrm{n}}$ is the position of the transmitter at the time of transmitting the $k^{\text {th }}$ pulse expressed in the navigation frame $n, r_{m}^{\mathrm{n}}$ is the position of the $m^{\text {th }}$ receiver and $\Delta \tau_{m}$ is the clock-offset of the $m^{\text {th }}$ receiver. The UWB calibration algorithm estimates the parameter vector $\theta$, defined as

$$
\theta=\left(\left\{t_{k}^{\mathrm{n}}, \tau_{k}\right\}_{k=1}^{K},\left\{r_{m}^{\mathrm{n}}, \Delta \tau_{m}\right\}_{m=1}^{M}\right) .
$$

The choice of the noise distribution $e_{t}$ in (3.13) influences the likelihood function in (3.1) and hence the optimization problem (3.2). The most commonly used noise model assumes that $e_{t}$ is zero-mean Gaussian noise. The noise of the inertial sensors and the magnetometers can typically be assumed to be Gaussian (Titterton and Weston, 1997; Hol, 2011). Hence, the Gaussian cost function and its gradient and approximate Hessian as given in Example 3.1 are frequently used in Part II of this thesis.

For the UWB measurements considered in Paper B, however, we expect a small number of measurements to be delayed due to multipath and/or NLOS conditions. A Gaussian PDF is depicted in blue in the left plot in Figure 3.2. As can be seen, the likelihood far away from the mean is small and the log likelihood cost function (right plot) far away from the mean is large. Hence, the presence of outliers is not well described by a Gaussian distribution. In Paper B, we instead use a Cauchy distribution to allow for the presence of outliers. The Cauchy PDF 

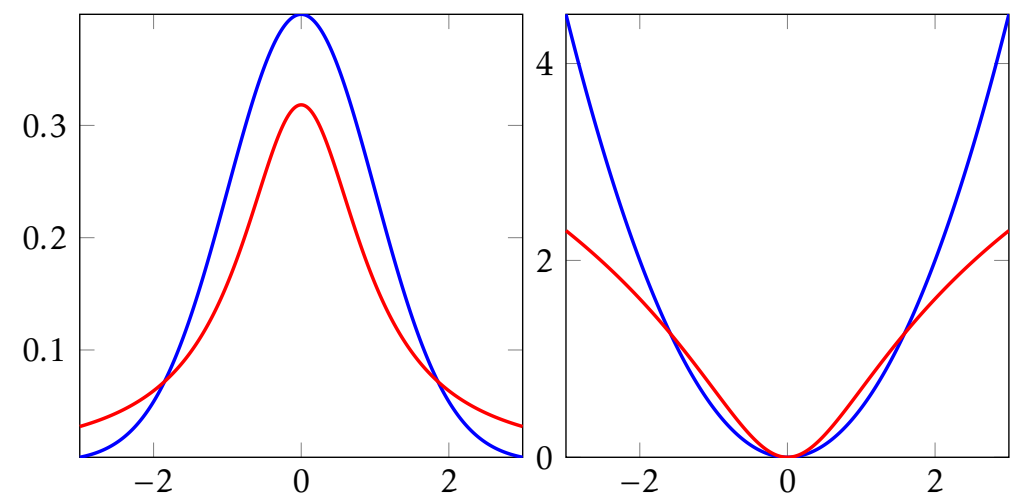

Figure 3.2: Left: PDF of a $\mathcal{N}(0,1)$ distribution (blue) and a Cauchy $(0,1)$ distribution (red). Right: $\log$ likelihood cost function of the PDF $\mathcal{N}(0,1)$ (blue) and of the PDF Cauchy $(0,1)$ (red).

and its log likelihood cost function are depicted in red in Figure 3.2. The PDF has heavy tails (left plot) and its log likelihood cost function (right plot) hence does not severely punish the presence of outliers. To only allow for time delays of the UWB pulses, in Paper B we assume that the noise is asymmetrically distributed, with a Cauchy distribution on one side and a Gaussian distribution on the other. In Example 3.3 we will illustrate the difference in handling outliers between a Gaussian and a Cauchy distribution.

Example 3.3: Parameter estimation with/without measurement outliers — Consider the problem of estimating the parameter $\theta$ in the following regression problem

$$
y_{t}=\theta t+e_{t}
$$

Assuming $e_{t} \sim \mathcal{N}(0,1)$ and $\theta=0.2$ we simulate the measurements depicted in Figure 3.3. In the left plot, 50 measurements $y_{t}$ are simulated at $t=-25, \ldots, 25$. In the right plot, we assume the presence of two outliers, $y_{t}=20$ at $t=-15$ and $y_{t}=-20$ at $t=15$. An estimate of the parameter $\theta$ is obtained both assuming a Gaussian $\left(\hat{\theta}^{\mathrm{G}}\right)$ and a Cauchy distribution $\left(\hat{\theta}^{\mathrm{C}}\right)$ for the noise $e_{t}$ (see Examples 3.1 and 3.2 , respectively). The lines drawn with the estimated parameters $\hat{\theta}^{\mathrm{G}}$ and $\hat{\theta}^{\mathrm{C}}$ show that the presence of the outliers has a greater impact on the estimate $\hat{\theta}^{\mathrm{G}}$ than on the estimate $\hat{\theta}^{\mathrm{C}}$. This is due to the heavy tails of the Cauchy distribution, as shown in Figure 3.2.

\subsection{Model parameters in a state-space model}

In (3.3) we described the magnetometer measurement function used in Paper A to account for the case that the magnetometer is uncalibrated. Comparing (3.3b) 

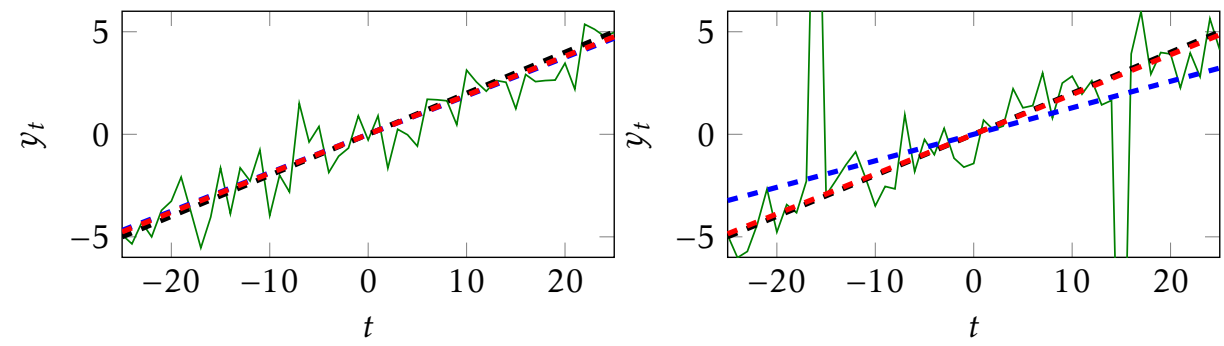

Figure 3.3: Measurements $y_{t}$ (green) with (dashed black) the line $\theta t$ (see (3.16)), (dashed blue) the line $\hat{\theta}_{G} t$ and (dashed red) $\hat{\theta}_{C} t$. Left: 50 measurements at $t=-25, \ldots, 25$. Right: 50 measurements at $t=-25, \ldots, 25$, but at $t=-15$ and $t=15$, we assume two measurement outliers $y_{t}=20$ and $y_{t}=-20$, respectively.

to (3.13), we note that there is a fundamental difference. The model (3.13) consists of only known quantities and model parameters. However, the model (3.3b) consists of both known quantities, model parameters and states $R_{t}^{\mathrm{nb}}$ from a statespace model (1.1).

To fully define the magnetometer calibration problem, we therefore need to include the state-space model and determine $D$ and $o$ from

$$
\begin{aligned}
R_{t+1}^{\mathrm{nb}} & =f_{t}\left(R_{t}^{\mathrm{nb}}, y_{\omega, t}, e_{\omega, t}\right), \\
y_{\mathrm{a}, t} & =-R_{t}^{\mathrm{bn}} g^{\mathrm{n}}+e_{\mathrm{a}, t}, \\
y_{\mathrm{m}, t} & =D R_{t}^{\mathrm{bn}} m^{\mathrm{n}}+o+e_{\mathrm{m}, t},
\end{aligned}
$$

where we denote the state vector representing the orientation as $R_{t}^{\mathrm{nb}}$. Note that we implicitly assume that the state is parametrized by either a quaternion as in Algorithm 1 or by an orientation deviation from a linearization point as in Algorithm 2.

Equivalently to (3.4), we want to estimate the predicted measurements $\hat{y}_{t}(\theta)$ from the model. The predicted measurements in (3.17), however, depend on the states. Hence, using conditional probabilities and the fact that the logarithm is a monotonic function, we have the following equivalent formulation of (3.2),

$$
\hat{\theta}^{\mathrm{ML}}=\underset{\theta \in \Theta}{\arg \min }-\sum_{t=1}^{N} \log p_{\theta}\left(y_{t} \mid y_{1: t-1}\right),
$$

where we use the convention that $y_{1: 0} \triangleq \emptyset$. In case the state-space model is nonlinear, there is typically no closed form solution available for the one step ahead predictor $p_{\theta}\left(y_{t} \mid y_{1: t-1}\right)$ in (3.18). In Paper A, we assume that the noise is Gaussian and approximate the one step ahead predictor using an EKF, see also Section 2.2. The result is

$$
p_{\theta}\left(y_{t} \mid y_{1: t-1}\right) \approx \mathcal{N}\left(y_{t} \mid \hat{y}_{t \mid t-1}(\theta), S_{t}(\theta)\right)
$$


where, $\hat{y}_{t \mid t-1}$ and $S_{t}$ are estimated using an EKF. Inserting (3.19) into (3.18) and neglecting all constants results in the following optimization problem,

$$
\min _{\theta \in \Theta} \frac{1}{2} \sum_{t=1}^{N}\left(\left\|y_{t}-\hat{y}_{t \mid t-1}(\theta)\right\|_{S_{t}^{-1}(\theta)}^{2}+\log \operatorname{det} S_{t}(\theta)\right) .
$$

As discussed in Section 3.1, we solve our optimization problems using the gradient and the (approximate) Hessian of the cost function. For the cases discussed in Section 3.2, these expressions can be obtained relatively easy as shown in Examples 3.1 and 3.2. For the cost function (3.20), however, it is less straightforward to obtain an expression for the gradient and the Hessian. Using the time and measurement update equations (2.9) and (2.11) for a scalar parameter $\theta$, the Jacobian can be derived to be

$$
\begin{aligned}
\frac{\partial \hat{y}_{t+1 \mid t}}{\partial \theta} & =C_{t} \frac{\partial \hat{x}_{t+1 \mid t}}{\partial \theta}+\frac{\partial C_{t}}{\partial \theta} \hat{x}_{t+1 \mid t} \\
\frac{\partial \hat{x}_{t+1 \mid t}}{\partial \theta} & =\frac{\partial A_{t}}{\partial \theta} \hat{x}_{t \mid t}+A_{t} \frac{\partial \hat{x}_{t \mid t}}{\partial \theta} \\
\frac{\partial \hat{x}_{t \mid t}}{\partial \theta} & =\frac{\partial \hat{x}_{t \mid t-1}}{\partial \theta}+\frac{\partial}{\partial \theta}\left(P_{t \mid t-1} C_{t}^{\top}\left(C_{t} P_{t \mid t-1} C_{t}^{\top}+R\right)^{-1}\left(y_{t}-\hat{y}_{t \mid t-1}\right)\right) \\
\frac{\partial P_{t \mid t-1}}{\partial \theta} & =\frac{\partial}{\partial \theta}\left(A_{t-1} P_{t-1 \mid t-1} A_{t-1}^{\top}+G_{t-1} Q G_{t-1}^{\top}\right)
\end{aligned}
$$

As can be seen from (3.21), the expression for the gradient of the objective function in (3.20) is defined recursively using the EKF time and measurement equations. Its computation is hence more involved than in the case discussed in Section 3.2. In Åström (1980); Segal and Weinstein (1989), different approaches are discussed to determine analytical gradients of the objective function in (3.20). They, however, consider the case of a linear state-space model. In our problems, we use an EKF implementation where the matrices $A_{t}, C_{t}$ and $G_{t}$ are based on linear approximations of the dynamic and measurement model. Hence, these methods only lead to approximate gradients of the objective function. Because of this, in Paper A we solve (3.20) using numerical gradients and a Hessian estimated using a BFGS algorithm. This approach is computationally quite expensive, but the computations of the gradients can easily be parallelized. 


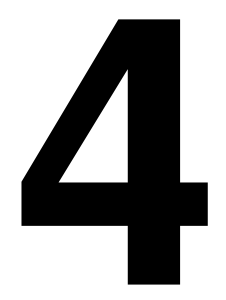

\section{Concluding remarks}

In Part I of this thesis, we have given an introduction to the four papers that will be presented in Part II. In Chapter 1, the different sensors that are used throughout the thesis were introduced together with the subject of probabilistic modeling. Chapter 2 subsequently focused on pose estimation using inertial sensors and magnetometers, introducing relevant background material for Papers A - D in Part II. The subject of sensor calibration was discussed in Chapter 3. It provides background to the magnetometer calibration problem discussed in Paper A and the calibration of the UWB setup in Paper B. In this chapter, we will summarize the contributions of this thesis in Section 4.1 and discuss possible directions for future work in Section 4.2.

\subsection{Summary of the contributions}

The main contributions of the thesis are within the domain of position and orientation (pose) estimation using inertial sensors in combination with additional (sensor) information. Since using uncalibrated sensors for pose estimation would lead to inaccurate estimates, we focus both on pose estimation algorithms and on sensor calibration. The problems are formulated based on probabilistic models of the sensor information and the model assumptions. The pose estimation algorithms are solved using MAP estimation while the calibration problems are solved using ML algorithms.

\subsubsection{Sensor calibration}

Sensor calibration problems are addressed in Papers A and B. In Paper A, we consider the problem of combining the inertial sensors with a magnetometer for orientation estimation. The magnetometer is not assumed to be calibrated. We 
present a practical calibration algorithm that calibrates the magnetometer for the presence of magnetic disturbances rigidly attached to the sensor, for magnetometer sensor errors and for misalignment between the inertial and the magnetometer sensor axes. Using the calibrated magnetometer measurements to estimate the sensor's orientation is experimentally shown to lead to significantly improved heading estimates.

Paper B focuses both on calibration and on state estimation. It addresses the problem of combining TOA measurements from a UWB system with inertial measurements for $6 \mathrm{D}$ pose estimation. We use a setup where a number of UWB receivers are placed in an indoor environment and move a UWB transmitter through the measurement volume. To be able to obtain position information from the UWB measurements, it is imperative that accurate estimates of the receivers' positions and clock offsets are known. To avoid the typically labor-intensive and timeconsuming process of surveying the receivers' positions, we present an easy-touse calibration method. We model the UWB measurements assuming an asymmetric heavy-tailed noise distribution, which naturally handles measurement outliers due to multipath and/or NLOS conditions.

\subsubsection{Pose estimation}

After the UWB calibration discussed in Section 4.1.1, the UWB system considered in Paper B can be used for pose estimation of a subject wearing inertial sensors and UWB transmitters walking through the environment. We present a tightlycoupled sensor fusion approach to combine the inertial measurements with the TOA measurements. It is shown to lead to accurate pose estimates as compared to data from an optical reference system.

Paper C discusses the problem of inertial human body motion capture, where a multitude of body segments are equipped with IMUs as shown in Figure 1.5. Relative position and orientation estimates are obtained using the inertial measurements together with a biomechanical model, which models the body in terms of connected body segments. The problem is formulated as a MAP problem and is solved using optimization-based techniques. As a proof-of-concept we apply our algorithm to a lower body configuration, illustrating that the estimates are drift-free and match the joint angles from an optical reference system.

Paper D presents an approach for 6D pose estimation where inertial measurements are complemented with magnetometer measurements assuming that a model (map) of the magnetic field is available. In our experimental study, the magnetic field is generated by a magnetic coil, giving rise to a magnetic field that we can model analytically. The experimental results show that accurate position estimates can be obtained in the vicinity of the coil, where the magnetic field is strong. 


\subsection{Future work}

In this section we will discuss ideas for future work for each of the papers. In Section 4.2.1 we will first discuss ideas for future work in the field of sensor calibration, related to Paper A. Subsequently, in Section 4.2.2 we will first discuss ideas for future work in the field of pose estimation, related to Papers B - D.

\subsubsection{Sensor calibration}

In Paper A we show that our calibration algorithm leads to significantly improved heading estimates based on measurements from two different commercially available IMUs. An interesting line of future work is to apply the magnetometer calibration algorithm to inertial and magnetometer measurements from a smartphone. Smartphones typically use their own magnetometer calibration algorithm, thereby complicating the testing of other calibration algorithms. However, as of Android API level 18 (Jelly Bean MR2), it is possible to log uncalibrated magnetometer data. Hence, it is be possible to apply our calibration algorithm to measurements from a smartphone.

Another possible direction for future work extends the calibration algorithm to also be able to include GPS measurements in outdoor applications. In that case, the extended Kalman filter (EKF) providing the measurement predictions to the ML problem as discussed in Section 3.3 would have to be extended to include at least a position and a velocity state. The additional GPS information should significantly help in calibrating the magnetometer. However, the algorithm would be computationally more expensive due to the additional states in the EKF.

The calibration algorithm is now formulated as a batch, offline, method. It would be interesting to extend it to an online approach. Using this approach, it might be possible to automatically recalibrate the sensor once it enters a different magnetic environment.

\subsubsection{Pose estimation}

Paper B uses a heavy-tailed asymmetric noise distribution to represent the presence of outliers in the UWB measurements. This distribution is used both in the UWB calibration algorithm and in our approach to determine the transmitter's position using UWB multilateration. In future work we are planning to extend the sensor fusion algorithm which combines the UWB measurements with inertial measurements to also make use of the heavy-tailed asymmetric noise distribution. We plan to implement the sensor fusion algorithm as an optimization problem similar to our approach in Paper C. Using an optimization formulation, different noise assumptions can straightforwardly be used.

In Paper $\mathrm{C}$, we apply our motion capture algorithm to a lower body configuration consisting of 7 IMUs places on the feet, lower legs, upper legs and pelvis. An obvious direction of future work would of course be to include more body segments. 
The algorithm relies on knowledge about the position and orientation of the sensors on the body. This information can be regarded as calibration parameters. We plan to extend the approach to also estimate these calibration parameters.

In Paper $\mathrm{C}$, we focus only on estimating body's relative pose. To estimate its absolute position, it is possible to include foot step detection, see e.g. Callmer (2013). It would also be possible to combine the approaches in Papers B and C and use UWB measurements to estimate the position of the body.

Paper D discusses the problem of pose estimation assuming a known magnetic field map. In experiments, we have used a magnetic coil to generate a known magnetic field. We have also been working on an approach to estimate the magnetic field map (Wahlström et al., 2013). An interesting line of research would be to combine both approaches. The ultimate goal would then be to do simultaneous localization and mapping (SLAM) where we simultaneously build a magnetic map of the environment and localize the sensor in the environment. 


\section{Bibliography}

M. Angermann, M. Frassl, M. Doniec, B. J. Julian, and P. Robertson. Characterization of the indoor magnetic field for applications in localization and mapping. In Proceedings of the IEEE International Conference on Indoor Positioning and Indoor Navigation (IPIN), pages 1-9, Sydney, Australia, Nov 2012.

K. J. Åström. Maximum likelihood and prediction error methods. Automatica, 16(5):551-574, 1980.

C. M. Bishop. Pattern recognition and machine learning. Springer, 2006.

T. Bohlin. Practical Grey-box Process Identification; Theory and Appications. Springer, 2006.

S. Boyd and L. Vandenberghe. Convex Optimization. Cambridge University Press, 2004.

J. Callmer. Autonomous Localization in Unknown Environments. PhD thesis, Linköping University, Sweden, June 2013.

J. L. Crassidis, F. L. Markley, and Y. Cheng. A survey of nonlinear attitude estimation methods. Journal of Guidance, Control, and Dynamics, 30(1):12-28, 2007.

A. Doucet and A. M. Johansen. A tutorial on particle filtering and smoothing: Fifteen years later. In The Oxford Handbook of Nonlinear Filtering. Oxford University Press, 2011.

H. Driessen and Y. Boers. MAP estimation in particle filter tracking. In IET Seminar on Target Tracking and Data Fusion: Algorithms and Applications, pages $41-45,2008$.

M. Frassl, M. Angermann, M. Lichtenstern, P. Robertson, B. J. Julian, and M. Doniec. Magnetic maps of indoor environments for precise localization of legged and non-legged locomotion. In Proceedings of the IEEE/RSJ International Conference on Intelligent Robots and Systems (IROS), pages 913-920, Tokyo, Japan, Nov 2013. 
N. J. Gordon, D. J. Salmond, and A. F. M. Smith. Novel approach to nonlinear/non-Gaussian Bayesian state estimation. In IEE Proceedings on Radar and Signal Processing, volume 140, pages 107-113, 1993.

S. Grzonka. Mapping, State Estimation, and Navigation for Quadrotors and Human-Worn Sensor Systems. PhD thesis, Freiburg University, Germany, September 2011.

F. Gustafsson. Statistical Sensor Fusion. Studentlitteratur, 2012.

W. R. Hamilton. On quaternions; or on a new system of imaginaries in algebra. Philosophical Magazine, xxv, 1844.

J. D. Hol. Sensor Fusion and Calibration of Inertial Sensors, Vision, UltraWideband and GPS. PhD thesis, Linköping University, Sweden, June 2011.

J. D. Hol, F. Dijkstra, H. Luinge, and T. B. Schön. Tightly coupled UWB/IMU pose estimation. In Proceedings of the IEEE International Conference on UltraWideband (ICUWB), pages 688-692, Vancouver, Canada, September 2009.

J. D. Hol, T. B. Schön, and F. Gustafsson. Ultra-wideband calibration for indoor positioning. In Proceedings of the IEEE International Conference on UltraWideband (ICUWB), pages 1-4, Nanjing, China, September 2010.

R. E. Kalman. A new approach to linear filtering and prediction problems. Journal of basic Engineering, 82(1):35-45, 1960.

M. Kok and T. B. Schön. Maximum likelihood calibration of a magnetometer using inertial sensors. In Proceedings of the 19th World Congress of the International Federation of Automatic Control (accepted for publication), Cape Town, South Africa, August 2014a.

M. Kok and T. B. Schön. Magnetometer calibration using inertial sensors. Preprint, 2014b.

M. Kok, J. D. Hol, T. B. Schön, F. Gustafsson, and H. Luinge. Calibration of a magnetometer in combination with inertial sensors. In Proceedings of the 15th International Conference on Information Fusion, Singapore, July 2012.

M. Kok, N. Wahlström, T. B. Schön, and F. Gustafsson. MEMS-based inertial navigation based on a magnetic field map. In Proceedings of the 38th International Conference on Acoustics, Speech, and Signal Processing (ICASSP), pages 64666470, Vancouver, Canada, May 2013.

M. Kok, J. D. Hol, and T. B. Schön. An optimization-based approach to human body motion capture using inertial sensors. In Proceedings of the 19th World Congress of the International Federation of Automatic Control (accepted for publication), Cape Town, South Africa, August 2014a.

M. Kok, J. D. Hol, and T. B. Schön. Indoor positioning using ultra-wideband and inertial measurements. Preprint, 2014b. 
J. Kronander, J. Dahlin, D. Jönsson, M. Kok, T. B. Schön, and J. Unger. Real-time video based lighting using GPU raytracing. In Proceedings of the 2014 European Signal Processing Conference (EUSIPCO), Lisbon, Portugal, September 2014. (submitted, pending review).

J. B. Kuipers. Quaternions and Rotation Sequences: a primer with applications to orbits, aerospace, and virtual reality. Princeton University Press, 1999.

F. Lindsten. Particle filters and Markov chains for learning of dynamical systems. PhD thesis, Linköping University, Sweden, 2013.

F. Lindsten and T. B. Schön. Backward simulation methods for Monte Carlo statistical inference. Foundations and Trends in Machine Learning, 6(1):1-143, 2013.

L. Ljung. System Identification, Theory for the User. Prentice Hall PTR, 2nd edition, 1999.

H. J. Luinge. Inertial Sensing of Human Movement. PhD thesis, University of Twente, Enschede, the Netherlands, October 2002.

F. L. Markley. Attitude error representations for Kalman filtering. Journal of guidance, control, and dynamics, 26(2):311-317, 2003.

J. Nocedal and S. J. Wright. Numerical Optimization. Springer Series in Operations Research, 2nd edition, 2006.

J. B. Rawlings and D. Q. Mayne. Model Predictive Control: Theory and Design. Nob Hill Publishing, 2009.

S. Saha, Y. Boers, H. Driessen, P. K. Mandal, and A. Bagchi. Particle based MAP state estimation: A comparison. In Proceedings of the 12th International Conference on Information Fusion, pages 278-283, July 2009.

S. Saha, P. Mandal, A. Bagchi, Y. Boers, and H. Driessen. Particle based smoothed marginal MAP estimation for general state space models. IEEE Transactions on Signal Processing, 61(2):264-273, 2013.

T. B. Schön, F. Gustafsson, and P. J. Nordlund. Marginalized particle filters for mixed linear/nonlinear state-space models. IEEE Transactions on Signal Processing, 53(7):2279-2289, 2005.

M. Segal and E. Weinstein. A new method for evaluating the log-likelihood gradient, the Hessian, and the Fisher information matrix for linear dynamic systems. IEEE Transactions on Information Theory, 35(3):682-687, 1989.

M. D. Shuster. A survey of attitude representations. The Journal of the Astronautical Sciences, 41(4):439-517, oct-dec 1993.

D. H. Titterton and J. L. Weston. Strapdown inertial navigation technology. IEE radar, sonar, navigaton and avionics series 5. Peter Peregrinus LTd. on behalf of the Institution of Electrical Engineers, 1997. 
D. Törnqvist. Estimation and Detection with Applications to Navigation. PhD thesis, Linköping University, November 2008. Thesis No. 1216.

N. Wahlström, M. Kok, T. B. Schön, and F. Gustafsson. Modeling magnetic fields using Gaussian processes. In Proceedings of the 38th International Conference on Acoustics, Speech, and Signal Processing (ICASSP), pages 3522-3526, Vancouver, Canada, May 2013.

O. J. Woodman. Pedestrian localisation for indoor environments. PhD thesis, University of Cambridge, United Kingdom, September 2010.

Xsens Technologies B.V. http: / /www.xsens. com, Accessed on April 24, 2014. 


\section{Part II}

\section{Publications}





\section{Paper D}

\section{MEMS-based inertial navigation based on a magnetic field map}

Authors: $\quad$ Manon Kok, Niklas Wahlström, Thomas B. Schön, Fredrik Gustafsson

Edited version of the paper:

M. Kok, N. Wahlström, T. B. Schön, and F. Gustafsson. MEMS-based inertial navigation based on a magnetic field map. In Proceedings of the 38th International Conference on Acoustics, Speech, and Signal Processing (ICASSP), pages 6466-6470, Vancouver, Canada, May 2013. 



\title{
MEMS-based inertial navigation based on a magnetic field map
}

\author{
Manon Kok ${ }^{\star}$, Niklas Wahlström${ }^{\star}$, Thomas B. Schön ${ }^{\dagger}$, Fredrik Gustafsson ${ }^{\star}$ \\ ${ }^{\star}$ Dept. of Electrical Engineering, \\ Linköping University, \\ SE-581 83 Linköping, Sweden \\ \{manko, nikwa, fredrik\} @isy.liu.se \\ ${ }^{\dagger}$ Dept. of Information Technology \\ Uppsala University, \\ SE-751 05 Uppsala, Sweden \\ thomas.schoneuu.se
}

\begin{abstract}
This paper presents an approach for 6D pose estimation where MEMS inertial measurements are complemented with magnetometer measurements assuming that a model (map) of the magnetic field is known. The resulting estimation problem is solved using a Rao-Blackwellized particle filter. In our experimental study the magnetic field is generated by a magnetic coil giving rise to a magnetic field that we can model using analytical expressions. The experimental results show that accurate position estimates can be obtained in the vicinity of the coil, where the magnetic field is strong.
\end{abstract}

\section{Introduction}

With the reducing cost of accelerometers and gyroscopes (inertial sensors) and magnetometers, these sensor are becoming increasingly available in day-to-day life. It is for instance common that these sensors are present in modern smartphones. Positioning based on inertial sensors alone suffers greatly from drift and does not give reliable estimates for any but the highest quality sensors. Because of this, sensors such as GPS and ultra-wideband are often used as an aiding source (Hol, 2011). While GPS solutions only work for outdoor applications, indoor solutions are often highly dependent on additional infrastructure.

Magnetometers are a reliable source of information due to their high sampling rates and reliable sensor readings. They measure the superposition of the local earth magnetic field and the magnetic field induced by magnetic structures in the vicinity. Magnetometers are widely used as a source of heading information, relying on the assumption that no magnetic disturbances are present. Especially in indoor applications this assumption is often violated due to the presence of steel in the construction of buildings and objects like radiators, tables and chairs.

This paper presents a method to obtain accurate position and orientation estimates based on inertial and magnetometer data assuming a map of the magnetic 
field is known. This enables positioning with widely available sensors, without requirements on additional infrastructure.

In recent years, the idea of using the presence of magnetic disturbances as a source of position information has started appearing in the literature. Most interest is from the robot localization perspective where odometry information is available (Suksakulchai et al., 2000; Navarro and Benet, 2009; Vallivaara et al., 2011; Georgiou and Dai, 2010). Generally, in these applications localization is only considered in $2 \mathrm{D}$, and the sensor is assumed to be rotating around only one axis. To the best of the authors' knowledge, little work has been done on combining inertial and magnetometer measurements, for example Vissière et al. (2007); Dorveaux et al. (2011). This is a more challenging problem compared to using odometry information, since low grade inertial measurement units (IMUs) generally have poor dead-reckoning performance. The approach presented in Vissière et al. (2007) is not based on magnetic field maps, but uses knowledge about the physical properties of the magnetic field and its gradient to aid localization using an extended Kalman filter approach. Other approaches focus on using sensors in smartphones for localization (Chung et al., 2011; IndoorAtlas, 2012; Gozick et al., 2011) and consider magnetometer data only or very limited information from the inertial sensors. The direction of the magnetic field can, however, only be derived from the magnetic field measurements when the sensor orientation is known. Not estimating the full orientation therefore poses constraints on the allowed sensor rotations. In our approach no constraints on the sensor rotations are required since the full $6 \mathrm{D}$ pose is estimated.

To isolate the problem of localization inside a known magnetic field map from the problem of obtaining the map, this work assumes that the magnetic field map is known and is generated by a magnetic coil. The reason for using a magnetic coil is that it is one of the few cases for which the magnetic field can be computed analytically. In other words, we have a perfect model describing the magnetic field produced by the magnetic coil. The magnetic field measurements can be described as a nonlinear function of the sensor position in this map and its orientation with respect to the map.

\section{Models}

Before introducing the dynamic and measurement equations, the relevant coordinate frames and the state vector will be introduced. All measurements are assumed to be obtained in the body coordinate frame denoted by $b$, which is the coordinate frame of the measurement unit with the origin in the center of the accelerometer triad. The position is tracked in the earth coordinate frame denoted by $e$, which is fixed in the world. The magnetic field map is represented in the map coordinate frame denoted by $m$ whose orientation is assumed to be aligned with that of the coil. The origin of the earth coordinate frame $e$ is assumed to coincide with that of the map coordinate frame and with the center of the magnetic coil. 


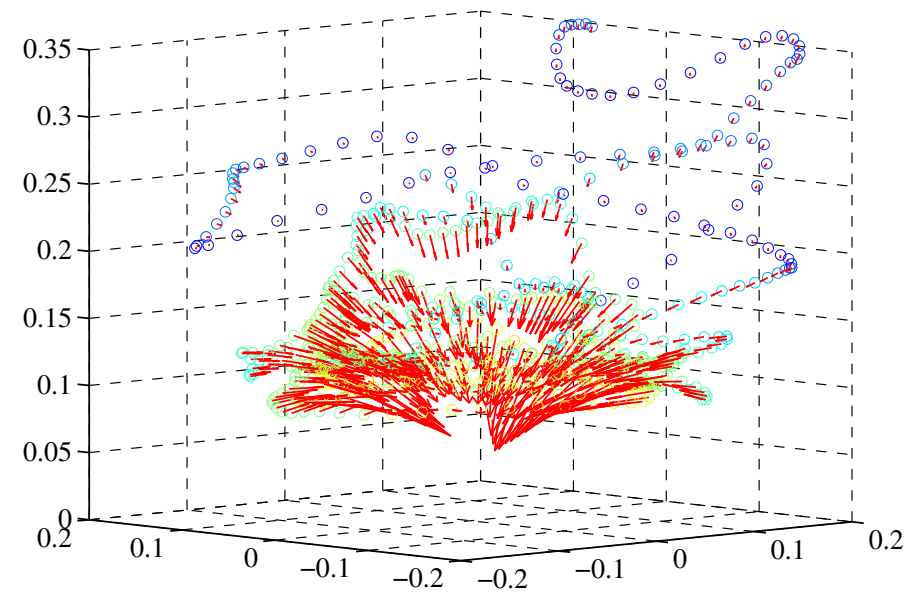

Figure 1: Magnetometer measurements represented in the earth coordinate frame. The measurements have been preprocessed by subtracting the earth magnetic field. The magnitude is indicated by the colors and the direction by the arrows.

The relevant state vector consists of the sensor's position $p^{\mathrm{e}}$ and velocity $v^{\mathrm{e}}$, its orientation with respect to the earth frame expressed as a unit quaternion $q^{\text {eb }}=$ $\left(\begin{array}{llll}q_{0} & q_{1} & q_{2} & q_{3}\end{array}\right)^{\top}$ and the gyroscope bias $b_{\omega}^{\mathrm{b}}$. In our model we have used the inertial measurements as inputs to the dynamic equations in order to not increase the state dimension. For reasons that will become clear after the model has been

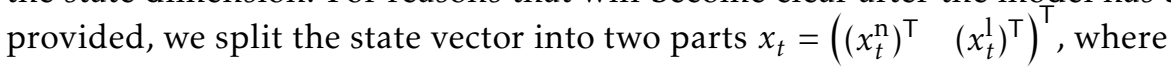

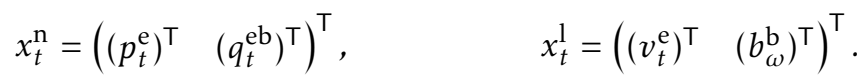

\subsection{Dynamical model}

The dynamical equations can be derived by using the inertial measurements as inputs. A commonly used, slowly time-varying random walk model is used for the gyroscope bias (Hol, 2011). This leads to the following state update equations 
for the linear and nonlinear states (Hol, 2011; Törnqvist, 2008)

$$
\begin{aligned}
& x_{t+1}^{\mathrm{n}}=\underbrace{\left(\begin{array}{cc}
\mathcal{I}_{3} & 0 \\
0 & \mathcal{I}_{4}
\end{array}\right)}_{A^{\mathrm{nn}}} x_{t}^{\mathrm{n}}+\underbrace{\left(\begin{array}{cc}
T \mathcal{I}_{3} & 0 \\
0 & -\frac{T}{2} \widetilde{S}\left(q_{t}^{\mathrm{eb}}\right)
\end{array}\right)}_{A_{t}^{\mathrm{nl}}\left(x_{t}^{\mathrm{n}}\right)} x_{t}^{1}+ \\
& \underbrace{\left(\begin{array}{ccc}
\frac{T^{2}}{2} \mathcal{R}\left(q_{t}^{\mathrm{eb}}\right) & \frac{T^{2}}{2} \mathcal{I}_{3} & 0 \\
0 & 0 & \frac{T}{2} \widetilde{S}\left(q_{t}^{\mathrm{eb}}\right)
\end{array}\right)}_{B_{t}^{\mathrm{n}}\left(x_{t}^{\mathrm{n}}\right)} u_{t}+\underbrace{\left(\begin{array}{cc}
\frac{T^{2}}{2} \mathcal{R}\left(q_{t}^{\mathrm{eb}}\right) & 0 \\
0 & \frac{T}{2} \widetilde{S}\left(q_{t}^{\mathrm{eb}}\right)
\end{array}\right)}_{G_{t}^{\mathrm{n}}\left(x_{t}^{\mathrm{n}}\right)} \underbrace{\left(\begin{array}{c}
w_{a, t}^{\mathrm{b}} \\
w_{\omega, t}^{\mathrm{b}}
\end{array}\right)}_{w_{t}^{\mathrm{n}}} \\
& x_{t+1}^{1}=\underbrace{\left(\begin{array}{cc}
\mathcal{I}_{3} & 0 \\
0 & \mathcal{I}_{3}
\end{array}\right)}_{A^{11}} x_{t}^{1}+\underbrace{\left(\begin{array}{ccc}
T \mathcal{R}\left(q_{t}^{\mathrm{eb}}\right) & T \mathcal{I}_{3} & 0 \\
0 & 0 & 0
\end{array}\right)}_{B_{t}^{1}\left(x_{t}^{\mathrm{n}}\right)} u_{t}+\underbrace{\left(\begin{array}{cc}
T \mathcal{R}\left(q_{t}^{\mathrm{eb}}\right) & 0 \\
0 & \mathcal{I}_{3}
\end{array}\right)}_{G_{t}^{1}\left(x_{t}^{\mathrm{n}}\right)} \underbrace{\left(\begin{array}{c}
w_{a, t}^{\mathrm{b}} \\
w_{\mathrm{b}_{\omega}, t}^{\mathrm{b}}
\end{array}\right)}_{w_{t}^{1}} .
\end{aligned}
$$

Here, $\mathcal{I}_{k}$ denotes the identity matrix of size $k \times k, \mathcal{R}\left(q_{t}^{\mathrm{eb}}\right) \in S O(3)$ is the rotation matrix obtained from the unit quaternion $q_{t}^{\mathrm{eb}}$ and ${ }^{1}$

$$
\widetilde{S}\left(q_{t}^{\mathrm{eb}}\right)=\left(\begin{array}{ccc}
-q_{1} & -q_{2} & -q_{3} \\
q_{0} & -q_{3} & q_{2} \\
q_{3} & q_{0} & -q_{1} \\
-q_{2} & q_{1} & q_{0}
\end{array}\right)
$$

The input vector $u_{t}$ is given by

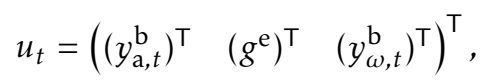

where $g^{\mathrm{e}}$ denotes the gravity vector and the accelerometer and the gyroscope measurements are denoted by $y_{\mathrm{a}}^{\mathrm{b}}$ and $y_{\omega}^{\mathrm{b}}$, respectively. The latter are modeled as

$$
\begin{aligned}
& y_{\mathrm{a}, t}^{\mathrm{b}}=R_{t}^{\mathrm{be}}\left(a_{t}^{\mathrm{e}}-g^{\mathrm{e}}\right)+w_{\mathrm{a}, t}^{\mathrm{b}}, \\
& y_{\omega, t}^{\mathrm{b}}=\omega_{t}^{\mathrm{b}}+b_{\omega}^{\mathrm{b}}+w_{\omega, t}^{\mathrm{b}},
\end{aligned}
$$

based on the fact that the accelerometer measures both the gravity vector and the body's free acceleration. The noise is modeled as

$$
\begin{aligned}
w_{\mathrm{a}}^{\mathrm{b}} & \sim \mathcal{N}\left(0, Q_{\mathrm{a}}\right), & Q_{\mathrm{a}} & =\sigma_{\mathrm{a}}^{2} \mathcal{I}_{3}, \\
w_{\omega}^{\mathrm{b}} & \sim \mathcal{N}\left(0, Q_{\omega}\right), & Q_{\omega} & =\sigma_{\omega}^{2} \mathcal{I}_{3}, \\
w_{\mathrm{b}_{\omega}}^{\mathrm{b}} & \sim \mathcal{N}\left(0, Q_{\mathrm{b}_{\omega}}\right), & Q_{\mathrm{b}_{\omega}} & =\sigma_{\mathrm{b}_{\omega}}^{2} \mathcal{I}_{3} .
\end{aligned}
$$

\footnotetext{
${ }^{1}$ Note that the propagation of the quaternion state in this way is an approximation, valid only for high sampling rates. The algorithm does not prevent use of the exact update equation and the approximation is only used to reduce computational complexity.
} 
The state noise is assumed to be distributed according to

$$
\begin{aligned}
w_{t} & =\left(\begin{array}{c}
w_{t}^{\mathrm{n}} \\
w_{t}^{1}
\end{array}\right) \sim \mathcal{N}(0, Q), \\
Q & =\left(\begin{array}{cc}
Q^{\mathrm{nn}} & Q^{\mathrm{nl}} \\
\left(Q^{\mathrm{nl}}\right)^{\top} & Q^{11}
\end{array}\right)=\left(\begin{array}{cc:cc}
Q_{\mathrm{a}} & 0 & Q_{\mathrm{a}} & 0 \\
0 & Q_{\omega} & 0 & 0 \\
\hdashline Q_{\mathrm{a}}^{\top} & 0 & Q_{\mathrm{a}} & 0 \\
0 & 0 & 0 & Q_{\mathrm{b}_{\omega}}
\end{array}\right) .
\end{aligned}
$$

Note that the linear and nonlinear state noise is highly correlated since the accelerometer noise acts on both the position and velocity states. This needs to be taken into account in the implementation.

\subsection{Magnetometer measurement model}

The magnetometer measurements are modeled as

$$
y_{\mathrm{m}, t}^{\mathrm{b}}=h\left(x_{t}^{\mathrm{n}}\right)+e_{\mathrm{m}, t}^{\mathrm{b}},
$$

where $e_{\mathrm{m}, t}^{\mathrm{b}} \sim \mathcal{N}(0, R)$ and $h\left(x_{t}^{\mathrm{n}}\right)$ is a function of the position $p_{t}^{\mathrm{e}}$ and orientation $q_{t}^{\mathrm{eb}}$ states. In practice this will be a superposition of the local earth magnetic field and all magnetic disturbances present.

As discussed in the introduction, to isolate the problem of positioning inside a map from the problem of making the map, we chose an experimental setup where the magnetic field is generated by a magnetic coil. In this case a magnetic field map is analytically known assuming the coil's position and orientation are known. The function $h\left(x_{t}^{\mathrm{n}}\right)$ is given by

$$
h\left(x_{t}^{\mathrm{n}}\right)=\mathcal{R}\left(q^{\mathrm{be}}\right) R^{\mathrm{em}} B\left(R^{\mathrm{me}} p_{t}^{\mathrm{e}}\right) .
$$

The function $B\left(R^{\mathrm{me}} p_{t}^{\mathrm{e}}\right)$ gives the magnetic field in the map coordinate frame at a position $p^{\mathrm{m}}$. The expression for the magnetic field from the coil is given by (Schepers, 2009)

$$
B\left(p^{\mathrm{m}}\right)=\frac{\mu_{0} N_{w} I}{2 \pi \sqrt{\left(\sqrt{p_{x}^{2}+p_{y}^{2}}+a\right)^{2}+p_{z}^{2}}}\left(\begin{array}{c}
\frac{p_{x} p_{z}}{p_{x}^{2}+p_{y}^{2}}\left[-K(k)+\frac{a^{2}+p_{x}^{2}+p_{y}^{2}+p_{z}^{2}}{\left(\sqrt{p_{x}^{2}+p_{y}^{2}}-a\right)^{2}+p_{z}^{2}} E(k)\right] \\
\frac{p_{y} p_{z}}{p_{x}^{2}+p_{y}^{2}}\left[-K(k)+\frac{a^{2}+p_{x}^{2}+p_{y}^{2}+p_{z}^{2}}{\left(\sqrt{p_{x}^{2}+p_{y}^{2}}-a\right)^{2}+p_{z}^{2}} E(k)\right] \\
{\left[K(k)+\frac{a^{2}-p_{x}^{2}-p_{y}^{2}-p_{z}^{2}}{\left(\sqrt{\left.p_{x}^{2}+p_{y}^{2}-a\right)^{2}+p_{z}^{2}} E(k)\right]}\right)}
\end{array}\right)
$$

where $p^{\mathrm{m}}=\left(\begin{array}{lll}p_{x} & p_{y} & p_{z}\end{array}\right), \mu_{0}$ is the magnetic permeability in vacuum, $a$ is the coil radius, $N_{w}$ is the number of windings, $I$ is the current through the coil and 
$E(k)$ and $K(k)$ are given by the following elliptic integrals

$$
\begin{aligned}
& E(k)=\int_{0}^{\pi / 2} \sqrt{1-k^{2} \sin ^{2} \theta} d \theta, \\
& K(k)=\int_{0}^{\pi / 2} \frac{1}{\sqrt{1-k^{2} \sin ^{2} \theta}} d \theta,
\end{aligned}
$$

where

$$
k=\sqrt{\frac{4 a \sqrt{p_{x}^{2}+p_{y}^{2}}}{\left(\sqrt{p_{x}^{2}+p_{y}^{2}}+a\right)^{2}+p_{z}^{2}}} .
$$

These equations implicitly assume that the origin of the earth coordinate frame coincides with that of the map coordinate frame. Note that our measurement model assumes that no background field is present.

\subsection{Some additional words about the magnetic field model}

The magnetic field of a coil is generally described as a function of the perpendicular distance $p_{z}$ towards the coil and the radial distance $r=\sqrt{p_{x}^{2}+p_{y}^{2}}$ towards the center of the coil (Schepers, 2009; Griffiths, 1999). However, in tracking we are interested in absolute position rather than just the distance to a source. Parametrizing the magnetic field in terms of a position $p_{x}, p_{y}, p_{z}$ introduces unobservability. Assuming the coil is placed horizontally, this results in two horizontal circles, one above and one below the coil, where the horizontal position is coupled to the heading as an unobservable manifold. We assume that the sensor can only be positioned above the coil and therefore have an entire circle of solutions at each time step. Note that in the more general case where multiple magnetic sources are present and possibly rotated with respect to each other, the unobservable manifold will be differently shaped or in some cases non-existent. To make our dynamic model applicable to any magnetic field map, we have not adapted the parametrization of our state vector to this specific structure.

\section{Computing the estimate}

As can be seen from the dynamical and measurement model presented in Section 2, the state dynamics is assumed to be linear while the measurement model is a nonlinear function of the sensor's position and orientation. A nonlinear filtering technique is therefore needed to compute a state estimate. A linear substructure can, however, be recognized, which can be exploited using a RaoBlackwellized particle filter (RBPF) in which the state is split into a state $x^{1}$ that enters linearly in both the dynamic and measurement model and a state $x^{\mathrm{n}}$ that 
enters non-linearly, where $x^{1}$ and $x^{\mathrm{n}}$ are defined by (1). An RBPF solves the nonlinear filtering problem by using a Kalman filter (KF) for the linear states and a particle filter (PF) for the nonlinear states.

The RBPF in this paper has been derived from Törnqvist (2008) and Lindsten (2011) and is summarized in Algorithm 1. It applies the model structure (2), (8), the noise assumptions (6) and their correlations given in (7). In (13), $\bar{x}_{t}^{i}$ and $\bar{P}_{t}^{i}$ are computed, which are a stacked version of the nonlinear and linear states and covariances. Based on these, the nonlinear and linear time update are given by (14), (15) respectively. Note that in (15) the pseudo-inverse, denoted by $t$, of $\bar{P}_{t}^{\mathrm{nn}, i}$ needs to be taken because this matrix is rank deficient due to the presence of quaternion states.

Since the measurement model (9) only depends on the nonlinear states, measurement information about the linear states is in our problem only available through the nonlinear states. Algorithm 1 does therefore not contain an explicit KF measurement update. However, measurement information implicitly present in the nonlinear states is taken into account in the linear states in (15).

\subsection{RBPF-MAP}

To compare particle filter estimates to reference data, a point estimate needs to be computed at each time step. The most commonly used approach for this is to take the conditional mean estimate. Due to the unobservability in our model (see Section 2.3), however, all particles on a horizontal circle are equally likely, which can lead to an uninformative point estimate in center of the circle.

In Driessen and Boers (2008); Saha et al. (2009) a maximum a posteriori estimate for the particle filter (PF-MAP) has been derived, which is argued to give a better point estimate in multi-modal applications. The PF-MAP estimate is an approximation of the MAP estimate given by

$$
\widehat{x}_{t \mid t}^{\mathrm{MAP}}=\underset{x_{t}^{i}}{\arg \max } p\left(y_{t} \mid x_{t}^{i}\right) \sum_{j} p\left(x_{t}^{i} \mid x_{1: t-1}^{j}\right) w_{t-1}^{j} .
$$

Following a similar reasoning, the RBPF-MAP estimate, can be shown to be

$$
\widehat{x}_{t \mid t}^{\mathrm{MAP}}=\underset{x_{t}^{\mathrm{n}, i}, x_{t}^{1, i}}{\arg \max } p\left(y_{t} \mid x_{t}^{\mathrm{n}, i}, x_{t}^{1, i}\right) \sum_{j} w_{t-1}^{j} \mathcal{N}\left(x_{t}^{i} ; \bar{x}_{t \mid t-1}^{j}, \bar{P}_{t \mid t-1}^{j}\right),
$$

where $\bar{x}_{t \mid t-1}^{j}$ and $\bar{P}_{t \mid t-1}^{j}$ can be obtained from (13). Note that since our problem does not have a KF measurement update, instead of the commonly used double subscript denoting the time for the linear states, Algorithm 1 only uses a single subscript.

When implementing this in Step 2 of the Algorithm 1, it needs to be taking into account that the covariance matrix $\bar{P}_{t}^{j}$ is rank deficient due to the presence of quaternion states. Because computation of (17) is computationally heavy, it could also be considered to use the most probable particle of the posterior. This would 


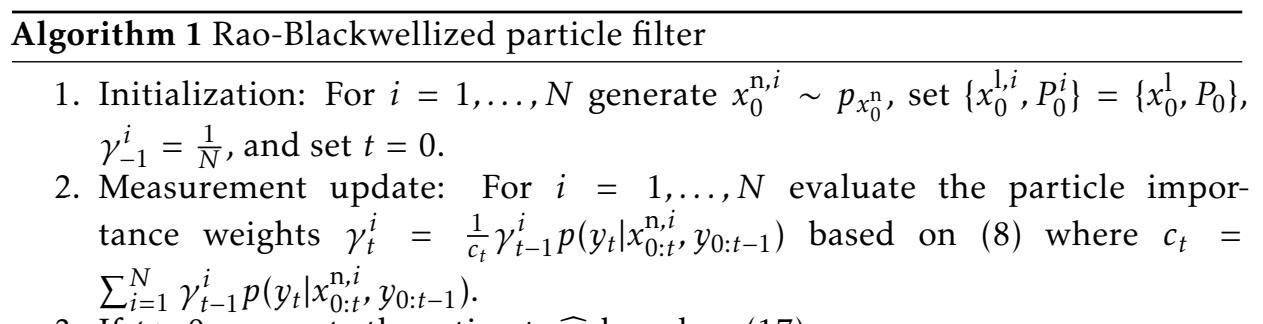

3. If $t>0$, compute the estimate $\widehat{x}_{t}$ based on (17).

4. Resampling: If $\widehat{N}_{\text {eff }}=\frac{1}{\sum_{i=1}^{N}\left(\gamma_{t}^{i}\right)^{2}}<\frac{2}{3} N$, resample $N$ particles with replacement from the set $\left\{x_{t}^{\mathrm{n}, i}, x_{t}^{1, i}\right\}_{i=1}^{N}$ where the probability to take sample $i$ is $\gamma_{t}^{i}$, and reset the weights to $\gamma_{t}^{i}=\frac{1}{N}$.

5. Time update: Determine the Gaussian mixture

where

$$
\begin{aligned}
& \bar{x}_{t+1}^{i}=A_{t}^{i} x_{t}^{i}+B_{t}^{i} u_{t}, \\
& \bar{P}_{t+1}^{i}=A_{t}^{1, i} P_{t}^{i}\left(A_{t}^{1, i}\right)^{\top}+G_{t}^{i} Q\left(G_{t}^{i}\right)^{\top},
\end{aligned}
$$

$$
\begin{aligned}
\bar{x}_{t}^{i} & =\left(\begin{array}{c}
\bar{x}_{t}^{\mathrm{n}, i} \\
\bar{x}_{t}^{1, i}
\end{array}\right), & \bar{P}_{t}^{i} & =\left(\begin{array}{cc}
\bar{P}_{t}^{\mathrm{nn}, i} & \bar{P}_{t}^{\mathrm{nl}, i} \\
\left(\bar{P}_{t}^{\mathrm{n}, i}\right)^{\mathrm{T}} & \bar{P}_{t}^{11, i}
\end{array}\right), \\
A_{t}^{1, i} & =\left(\begin{array}{c}
A_{t}^{\mathrm{nl}, i}\left(x_{t}^{\mathrm{n}, i}\right) \\
A^{1 \mathrm{l}}
\end{array}\right), & A_{t}^{i} & =\left(\begin{array}{cc}
A^{\mathrm{nn}} & A_{t}^{\mathrm{nl}, i}\left(x_{t}^{\mathrm{n}, i}\right) \\
0 & A^{\mathrm{ll}}
\end{array}\right), \\
B_{t}^{i} & =\left(\begin{array}{c}
B_{t}^{\mathrm{n}, i}\left(x_{t}^{\mathrm{n}, i}\right) \\
B_{t}^{1, i}\left(x_{t}^{\mathrm{n}, i}\right)
\end{array}\right), & G_{t}^{i} & =\left(\begin{array}{ccc}
G_{t}^{\mathrm{n}, i}\left(x_{t}^{\mathrm{n}, i}\right) & 0 \\
0 & G_{t}^{1, i}\left(x_{t}^{\mathrm{n}, i}\right)
\end{array}\right) .
\end{aligned}
$$

The nonlinear states can now sampled according to

$$
x_{t+1}^{\mathrm{n}, i} \sim \mathcal{N}\left(\bar{x}_{t+1}^{\mathrm{n}, i}, \bar{P}_{t+1}^{\mathrm{nn}, i}\right)
$$

and the linear states can be updated according to

$$
\begin{aligned}
& x_{t+1}^{1, i}=\bar{x}_{t+1}^{1, i}+\left(\bar{P}_{t+1}^{\mathrm{nl}, i}\right)^{\top}\left(\bar{P}_{t+1}^{\mathrm{nn}, i}\right)^{\dagger}\left(x_{t+1}^{\mathrm{n}, i}-\bar{x}_{t+1}^{\mathrm{n}, i}\right), \\
& P_{t+1}^{i}=\bar{P}_{t+1}^{1 \mathrm{ll}, i}-\left(\bar{P}_{t+1}^{\mathrm{nl}, i}\right)^{\top}\left(\bar{P}_{t+1}^{\mathrm{nn}, i}\right)^{\dagger} \bar{P}_{t+1}^{\mathrm{nl}, i} .
\end{aligned}
$$

6. Set $t:=t+1$ and iterate from Step 2 .

lead to similar results in Section 4.

\section{Experimental results}

\subsection{Experimental setup}

An experiment has been performed in which the magnetic field is generated by a magnetic coil where the number of windings $N_{w}$ is equal to 50 , the current $I$ through the coil is $1 \mathrm{~A}$ and the radius $a$ of the coil is $6 \mathrm{~cm}$. A MEMS IMU (Xsens MTi) providing synchronized inertial and magnetometer measurements at a sampling frequency of $100 \mathrm{~Hz}$ is used. A picture of the experimental setup can be found in Figure 2. Ground truth data is collected from an optical reference system (Vicon system) and is used for validation of the estimates as well as for 
determining the position and orientation $R^{\mathrm{em}}$ of the coil.

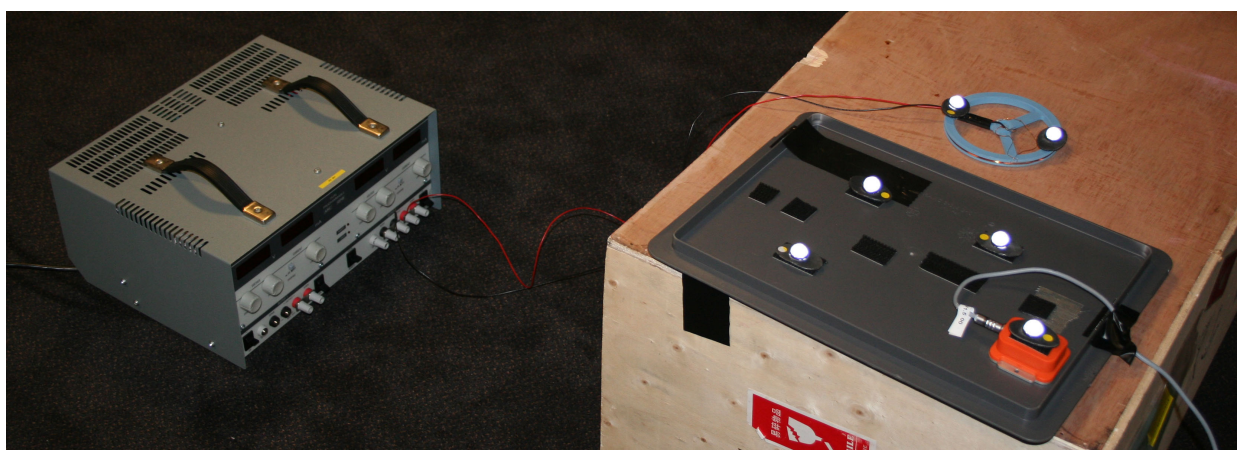

Figure 2: The experimental setup consisting of an IMU (orange box), a coil and a power supply. Optical markers are present, used for obtaining ground truth data, via an optical reference system.

Before the magnetometer measurements can be used in Algorithm 1, they need to be preprocessed for two reasons. First, the model (9) assumes that the magnetometer only measures the magnetic field due to a coil. A constant term representing the local earth magnetic field therefore needs to be determined and subtracted from all measurements. Second, the IMU used outputs magnetometer measurements in arbitrary units, while the model (9) determines the magnetic field in Tesla. A constant multiplication on all axes is therefore needed. Both constants are obtained by determining a best estimate from a part of the data where the magnetic disturbance is (approximately) zero. The preprocessed data is illustrated in Figure 1. The circles represent the preprocessed magnetometer measurements, downsampled to $4 \mathrm{~Hz}$. The color of the circles represents the magnitude of the magnetic field. The magnetic field falls off cubically with distance which explains why the magnitude of the magnetic field is reduced quickly with distance from the coil. Each preprocessed measurement also gives rise to a red arrow indicating the direction of the magnetic field. The length of the arrows illustrates the magnitude.

\subsection{Results}

Using the collected inertial and magnetometer data, Algorithm 1 can be applied to obtain state estimates. Due to the fact that the magnitude of the magnetic field falls off cubically with distance, all results in this section are based on data no further away from the coil's origin than $40 \mathrm{~cm}$. These have been compared to the ground truth data from the reference system. This section focuses on analysis of the position estimates. Due to the unobservability discussed in Section 2.3 we do not expect exact matches between the RBPF estimates and the ground truth data. A good comparison of the quality of the estimates, however, are the radial position and height estimates. The error plots can be found in Figure 3. The RBPF is initialized around the true estimate using the reference data, but any 
other (reasonable) initialization will give comparable results.

As can be seen in Figure 3, very good position estimates are obtained. However, at approximately $42 \mathrm{~s}$, there is a big peak in both the radial position and the height errors. This can be explained by the fact that at this time instant, the sensor is the furthest away from the coil, almost $40 \mathrm{~cm}$. The approach presented in this work is thus able to obtain high accurate position estimates for longer times, only when the sensor remains close to the coil. This is a major limitation in using the magnetic field as a source of position information in the way presented in this paper. The further away from the magnetic disturbance the less informative the measurements become. Even though at $40 \mathrm{~cm}$ from the coil the signal to noise ratio is still good, tracking problems occur due to model errors. It is therefore important to have a good model of the magnetic field (Wahlström et al., 2013).
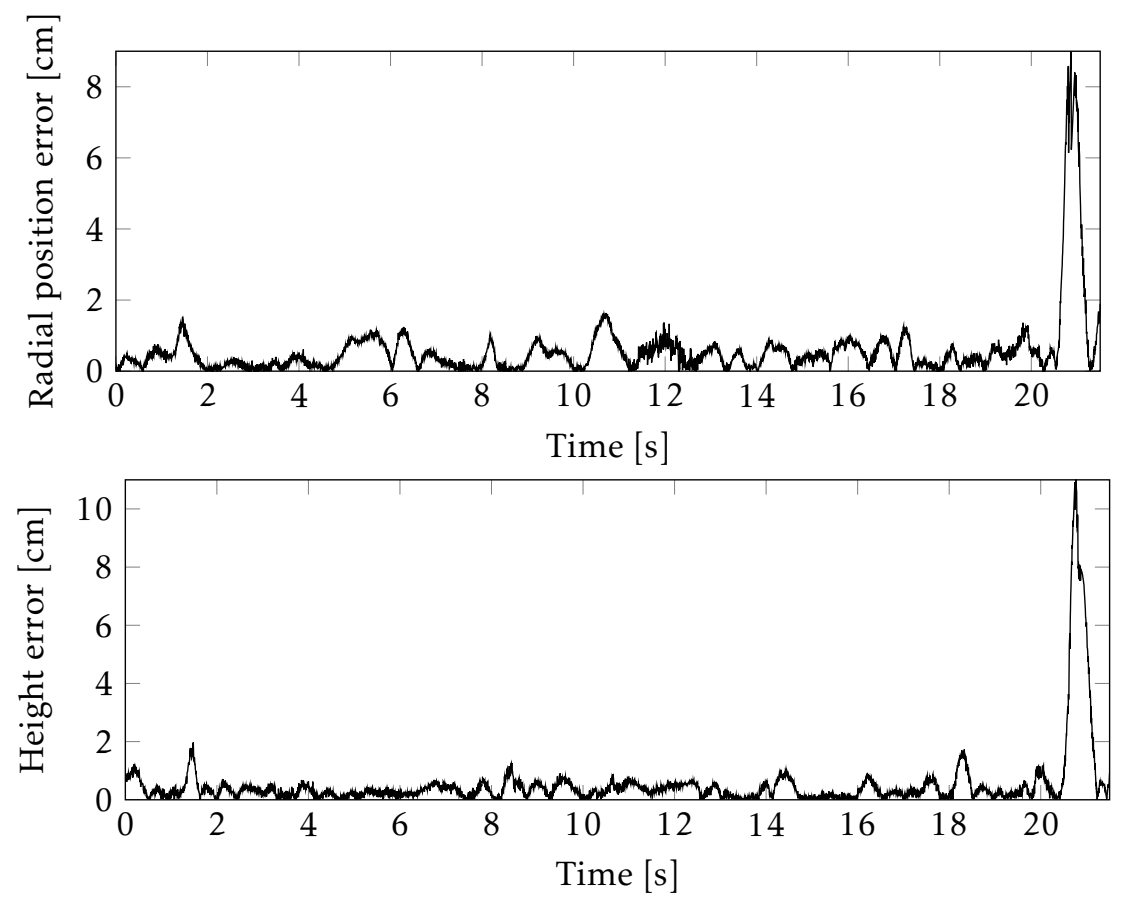

Figure 3: Error plots comparing the RBPF position estimates with the ground truth data from the optical reference system.

\section{Conclusions and future work}

This paper has shown that close to a magnetic distortion generated by a magnetic coil, good position and orientation estimates can be obtained from inertial and magnetometer data only. Ideas for future work include extending the magnetometer model to a more realistic measurement model. First trials show that 
we can probably deal with including the local earth magnetic field. We also aim at combining this work with Wahlström et al. (2013) into an approach where simultaneous localization and mapping (SLAM) is possible. Another future line of research aims at studying the unobservability manifolds from the magnetic field in different cases.

\section{Acknowledgements}

This work is supported by MC Impulse, a European Commission, FP7 research project and CADICS, a Linneaus Center funded by the Swedish Research Council (VR). The authors would like to thank Xsens Technologies for their support in starting this work as well as in collecting the data sets and Dr. Slawomir Grzonka for pointing out this interesting field of research. 


\section{Bibliography}

J. Chung, M. Donahoe, C. Schmandt, I. J. Kim, P. Razavai, and M. Wiseman. Indoor location sensing using geo-magnetism. In Proceedings of the 9 th international conference on Mobile systems, applications, and services (MobiSys), pages 141-154, Bethesda, USA, June 2011.

E. Dorveaux, T. Boudot, M. Hillion, and N. Petit. Combining inertial measurements and distributed magnetometry for motion estimation. In Proceedings of the American Control Conference (ACC), pages 4249-4256, San Francisco, USA, June 2011. IEEE.

H. Driessen and Y. Boers. MAP estimation in particle filter tracking. In IET Seminar on Target Tracking and Data Fusion: Algorithms and Applications, pages $41-45,2008$.

E. Georgiou and J. Dai. Self-localization of an autonomous maneuverable nonholonomic mobile robot using a hybrid double-compass configuration. In Proceedings of the 7th International Symposium on Mechatronics and its Applications (ISMA), pages 1-8, Sharjah, United Arab Emirates, April 2010. IEEE.

B. Gozick, K. P. Subbu, R. Dantu, and T. Maeshiro. Magnetic maps for indoor navigation. IEEE Transactions on Instrumentation and Measurement, 60(12): 3883-3891, 2011.

D. J. Griffiths. Introduction to electrodynamics, volume 3. Prentice Hall, New Jersey, 1999.

J. D. Hol. Sensor Fusion and Calibration of Inertial Sensors, Vision, UltraWideband and GPS. PhD thesis, Linköping University, Sweden, June 2011.

IndoorAtlas. http://www.indooratlas.com, Accessed on November 30, 2012.

M. Kok, N. Wahlström, T. B. Schön, and F. Gustafsson. MEMS-based inertial navigation based on a magnetic field map. In Proceedings of the 38th International Conference on Acoustics, Speech, and Signal Processing (ICASSP), pages 64666470, Vancouver, Canada, May 2013.

F. Lindsten. Rao-Blackwellised particle methods for inference and identification. Licentiate thesis, Linköping University, Sweden, 2011.

D. Navarro and G. Benet. Magnetic map building for mobile robot localization purpose. In Proceedings of the IEEE Conference on Emerging Technologies and Factory Automation (ETFA), pages 1-4, Mallorca, Spain, September 2009.

S. Saha, Y. Boers, H. Driessen, P. K. Mandal, and A. Bagchi. Particle based MAP state estimation: A comparison. In Proceedings of the 12th International Conference on Information Fusion, pages 278-283, July 2009.

H. M. Schepers. Ambulatory assessment of human body kinematics and kinetics. PhD thesis, University of Twente, Enschede, June 2009. 
S. Suksakulchai, S. Thongchai, D. M. Wilkes, and K. Kawamura. Mobile robot localization using an electronic compass for corridor environment. In Proceedings of the IEEE International Conference on Systems, Man, and Cybernetics (SMC), volume 5, pages 3354-3359, Nashville, USA, October 2000.

D. Törnqvist. Estimation and Detection with Applications to Navigation. PhD thesis, Linköping University, November 2008. Thesis No. 1216.

I. Vallivaara, J. Haverinen, A. Kemppainen, and J. Roning. Magnetic fieldbased SLAM method for solving the localization problem in mobile robot floorcleaning task. In Proceedings of the 15th International Conference on Advanced Robotics (ICAR), pages 198-203, Tallinn, Estonia, June 2011.

D. Vissière, A.P. Martin, and N. Petit. Using magnetic disturbances to improve IMU-based position estimation. In Proceedings of the European Control Conference (ECC), pages 2853-2858, Kos, Greece, July 2007.

N. Wahlström, M. Kok, T. B. Schön, and F. Gustafsson. Modeling magnetic fields using Gaussian processes. In Proceedings of the 38th International Conference on Acoustics, Speech, and Signal Processing (ICASSP), pages 3522-3526, Vancouver, Canada, May 2013. 


\section{Part II}

\section{Publications}

The articles associated with this thesis have been removed for copyright reasons. For more details about these see:

http://urn.kb.se/resolve?urn=urn:nbn:se:liu:diva-106882 


\section{Licentiate Theses \\ Division of Automatic Control \\ Linköping University}

P. Andersson: Adaptive Forgetting through Multiple Models and Adaptive Control of Car Dynamics. Thesis No. 15, 1983.

B. Wahlberg: On Model Simplification in System Identification. Thesis No. 47, 1985.

A. Isaksson: Identification of Time Varying Systems and Applications of System Identification to Signal Processing. Thesis No. 75, 1986.

G. Malmberg: A Study of Adaptive Control Missiles. Thesis No. 76, 1986.

S. Gunnarsson: On the Mean Square Error of Transfer Function Estimates with Applications to Control. Thesis No. 90, 1986.

M. Viberg: On the Adaptive Array Problem. Thesis No. 117, 1987.

K. Ståhl: On the Frequency Domain Analysis of Nonlinear Systems. Thesis No. 137, 1988.

A. Skeppstedt: Construction of Composite Models from Large Data-Sets. Thesis No. 149, 1988.

P. A. J. Nagy: MaMiS: A Programming Environment for Numeric/Symbolic Data Processing. Thesis No. 153, 1988.

K. Forsman: Applications of Constructive Algebra to Control Problems. Thesis No. 231, 1990.

I. Klein: Planning for a Class of Sequential Control Problems. Thesis No. 234, 1990.

F. Gustafsson: Optimal Segmentation of Linear Regression Parameters. Thesis No. 246, 1990.

H. Hjalmarsson: On Estimation of Model Quality in System Identification. Thesis No. 251, 1990.

S. Andersson: Sensor Array Processing; Application to Mobile Communication Systems and Dimension Reduction. Thesis No. 255, 1990.

K. Wang Chen: Observability and Invertibility of Nonlinear Systems: A Differential Algebraic Approach. Thesis No. 282, 1991.

J. Sjöberg: Regularization Issues in Neural Network Models of Dynamical Systems. Thesis No. 366, 1993.

P. Pucar: Segmentation of Laser Range Radar Images Using Hidden Markov Field Models. Thesis No. 403, 1993.

H. Fortell: Volterra and Algebraic Approaches to the Zero Dynamics. Thesis No. 438, 1994.

T. McKelvey: On State-Space Models in System Identification. Thesis No. 447, 1994.

T. Andersson: Concepts and Algorithms for Non-Linear System Identifiability. Thesis No. 448, 1994.

P. Lindskog: Algorithms and Tools for System Identification Using Prior Knowledge. Thesis No. 456, 1994.

J. Plantin: Algebraic Methods for Verification and Control of Discrete Event Dynamic Systems. Thesis No. 501, 1995.

J. Gunnarsson: On Modeling of Discrete Event Dynamic Systems, Using Symbolic Algebraic Methods. Thesis No. 502, 1995.

A. Ericsson: Fast Power Control to Counteract Rayleigh Fading in Cellular Radio Systems. Thesis No. 527, 1995.

M. Jirstrand: Algebraic Methods for Modeling and Design in Control. Thesis No. 540, 1996.

K. Edström: Simulation of Mode Switching Systems Using Switched Bond Graphs. Thesis No. 586, 1996. 
J. Palmqvist: On Integrity Monitoring of Integrated Navigation Systems. Thesis No. 600, 1997.

A. Stenman: Just-in-Time Models with Applications to Dynamical Systems. Thesis No. 601, 1997.

M. Andersson: Experimental Design and Updating of Finite Element Models. Thesis No. 611, 1997.

U. Forssell: Properties and Usage of Closed-Loop Identification Methods. Thesis No. 641, 1997.

M. Larsson: On Modeling and Diagnosis of Discrete Event Dynamic systems. Thesis No. 648, 1997.

N. Bergman: Bayesian Inference in Terrain Navigation. Thesis No. 649, 1997.

V. Einarsson: On Verification of Switched Systems Using Abstractions. Thesis No. 705, 1998.

J. Blom, F. Gunnarsson: Power Control in Cellular Radio Systems. Thesis No. 706, 1998.

P. Spångéus: Hybrid Control using LP and LMI methods - Some Applications. Thesis No. 724, 1998.

M. Norrlöf: On Analysis and Implementation of Iterative Learning Control. Thesis No. 727, 1998.

A. Hagenblad: Aspects of the Identification of Wiener Models. Thesis No. 793, 1999.

F. Tjärnström: Quality Estimation of Approximate Models. Thesis No. 810, 2000.

C. Carlsson: Vehicle Size and Orientation Estimation Using Geometric Fitting. Thesis No. 840, 2000.

J. Löfberg: Linear Model Predictive Control: Stability and Robustness. Thesis No. 866, 2001.

O. Härkegård: Flight Control Design Using Backstepping. Thesis No. 875, 2001.

J. Elbornsson: Equalization of Distortion in A/D Converters. Thesis No. 883, 2001.

J. Roll: Robust Verification and Identification of Piecewise Affine Systems. Thesis No. 899, 2001.

I. Lind: Regressor Selection in System Identification using ANOVA. Thesis No. 921, 2001.

R. Karlsson: Simulation Based Methods for Target Tracking. Thesis No. 930, 2002.

P.-J. Nordlund: Sequential Monte Carlo Filters and Integrated Navigation. Thesis No. 945, 2002.

M. Östring: Identification, Diagnosis, and Control of a Flexible Robot Arm. Thesis No. 948, 2002.

C. Olsson: Active Engine Vibration Isolation using Feedback Control. Thesis No. 968, 2002.

J. Jansson: Tracking and Decision Making for Automotive Collision Avoidance. Thesis No. 965, 2002.

N. Persson: Event Based Sampling with Application to Spectral Estimation. Thesis No. 981, 2002.

D. Lindgren: Subspace Selection Techniques for Classification Problems. Thesis No. 995, 2002.

E. Geijer Lundin: Uplink Load in CDMA Cellular Systems. Thesis No. 1045, 2003.

M. Enqvist: Some Results on Linear Models of Nonlinear Systems. Thesis No. 1046, 2003.

T. Schön: On Computational Methods for Nonlinear Estimation. Thesis No. 1047, 2003.

F. Gunnarsson: On Modeling and Control of Network Queue Dynamics. Thesis No. 1048, 2003.

S. Björklund: A Survey and Comparison of Time-Delay Estimation Methods in Linear Systems. Thesis No. 1061, 2003. 
M. Gerdin: Parameter Estimation in Linear Descriptor Systems. Thesis No. 1085, 2004.

A. Eidehall: An Automotive Lane Guidance System. Thesis No. 1122, 2004.

E. Wernholt: On Multivariable and Nonlinear Identification of Industrial Robots. Thesis No. 1131, 2004.

J. Gillberg: Methods for Frequency Domain Estimation of Continuous-Time Models. Thesis No. 1133, 2004.

G. Hendeby: Fundamental Estimation and Detection Limits in Linear Non-Gaussian Systems. Thesis No. 1199, 2005.

D. Axehill: Applications of Integer Quadratic Programming in Control and Communication. Thesis No. 1218, 2005.

J. Sjöberg: Some Results On Optimal Control for Nonlinear Descriptor Systems. Thesis No. 1227, 2006.

D. Törnqvist: Statistical Fault Detection with Applications to IMU Disturbances. Thesis No. 1258, 2006.

H. Tidefelt: Structural algorithms and perturbations in differential-algebraic equations. Thesis No. 1318, 2007.

S. Moberg: On Modeling and Control of Flexible Manipulators. Thesis No. 1336, 2007.

J. Wallén: On Kinematic Modelling and Iterative Learning Control of Industrial Robots. Thesis No. 1343, 2008.

J. Harju Johansson: A Structure Utilizing Inexact Primal-Dual Interior-Point Method for Analysis of Linear Differential Inclusions. Thesis No. 1367, 2008.

J. D. Hol: Pose Estimation and Calibration Algorithms for Vision and Inertial Sensors. Thesis No. 1370, 2008.

H. Ohlsson: Regression on Manifolds with Implications for System Identification. Thesis No. 1382, 2008.

D. Ankelhed: On low order controller synthesis using rational constraints. Thesis No. 1398, 2009.

P. Skoglar: Planning Methods for Aerial Exploration and Ground Target Tracking. Thesis No. 1420, 2009.

C. Lundquist: Automotive Sensor Fusion for Situation Awareness. Thesis No. 1422, 2009.

C. Lyzell: Initialization Methods for System Identification. Thesis No. 1426, 2009.

R. Falkeborn: Structure exploitation in semidefinite programming for control. Thesis No. 1430, 2010.

D. Petersson: Nonlinear Optimization Approaches to $\mathcal{H}_{2}$-Norm Based LPV Modelling and Control. Thesis No. 1453, 2010.

Z. Sjanic: Navigation and SAR Auto-focusing in a Sensor Fusion Framework. Thesis No. 1464, 2011.

K. Granström: Loop detection and extended target tracking using laser data. Thesis No. 1465, 2011.

J. Callmer: Topics in Localization and Mapping. Thesis No. 1489, 2011.

F. Lindsten: Rao-Blackwellised particle methods for inference and identification. Thesis No. 1480, 2011.

M. Skoglund: Visual Inertial Navigation and Calibration. Thesis No. 1500, 2011.

S. Khoshfetrat Pakazad: Topics in Robustness Analysis. Thesis No. 1512, 2011.

P. Axelsson: On Sensor Fusion Applied to Industrial Manipulators. Thesis No. 1511, 2011.

A. Carvalho Bittencourt: On Modeling and Diagnosis of Friction and Wear in Industrial Robots. Thesis No. 1516, 2012.

P. Rosander: Averaging level control in the presence of frequent inlet flow upsets. Thesis No. 1527, 2012. 
N. Wahlström: Localization using Magnetometers and Light Sensors. Thesis No. 1581, 2013.

R. Larsson: System Identification of Flight Mechanical Characteristics. Thesis No. 1599, 2013.

Y. Jung: Estimation of Inverse Models Applied to Power Amplifier Predistortion. Thesis No. 1605, 2013.

M. Syldatk: On Calibration of Ground Sensor Networks. Thesis No. 1611, 2013.

M. Roth: Kalman Filters for Nonlinear Systems and Heavy-Tailed Noise. Thesis No. 1613, 2013.

D. Simon: Model Predictive Control in Flight Control Design - Stability and Reference Tracking. Thesis No. 1642, 2014.

J. Dahlin: Sequential Monte Carlo for inference in nonlinear state space models. Thesis No. 1652, 2014. 